\title{
Design and Fabrication of Silicon Nanowires Towards Efficient Solar Cells
}

Peng Yu ${ }^{a}$, Jiang $W^{b}$, Shenting Liu $^{c}$, Jie Xiong ${ }^{c}$, Chennupati Jagadish ${ }^{d, *}$, and Zhiming M. Wang ${ }^{a, *}$

${ }^{a}$ Institute of Fundamental and Frontier Science, University of Electronic Science and Technology of China, Chengdu 610054, P.R. China

${ }^{\mathrm{b}}$ Department of Electronic and Electrical Engineering, University College London, Torrington Place, London WC1E 7JE, United Kingdom.

'State Key Laboratory of Electronic Thin Film and Integrated Devices, University of Electronic Science and Technology of China, Chengdu 610054, P.R. China

${ }^{\mathrm{d} D e p a r t m e n t ~ o f ~ E l e c t r o n i c ~ M a t e r i a l s ~ E n g i n e e r i n g, ~ R e s e a r c h ~ S c h o o l ~ o f ~ P h y s i c s ~ a n d ~ E n g i n e e r i n g, ~}$ The Australian National University, Canberra, ACT 2601, Australia.

*Corresponding author: zhmwang@uestc.edu.cn; Chennupati.Jagadish@anu.edu.au

\section{Abstract}

The recent rise of semiconductor nanowires opens new opportunities for realizing high efficiency photovoltaic devices at low cost due to the unique one-dimensional structure with remarkable electrical and optical properties. Particularly, silicon nanowires (SiNWs), as one of the most earthabundant materials, have been investigated worldwide to develop cost-effective solar cells. Great efforts have been devoted to fabricating ordered/disordered SiNWs using cost-effective approaches and achieving optimized structural parameters, such as array periodicity, nanowire morphology, length and diameter. Systematic theoretical investigations along with experimental studies on optical and electrical properties of SiNWs have been carried out. These efforts have led to obtaining remarkable improvement of the power conversion efficiency of SiNW solar cells from $<1 \%$ to $>10 \%$ in the last few years. However, till now, the power conversion efficiency of these SiNW solar cells is far from satisfactory for any commercial applications compared with the traditional bulk silicon solar cells. Further development of SiNW solar cells requires better understanding of the optical and electrical properties of the nanowire solar cells. Improvement in fabrication of high quality nanowires in a controlled fashion also plays a significant role in nanowire solar cell design and fabrication. To guide future development of SiNW solar cells, the recent work on SiNWs is reviewed. Following that, various techniques aiming to achieve high quality nanowires at low cost are introduced. Both bottom-up and top-down techniques are discussed. Then, electrical properties and various types of solar cells based on SiNWs are discussed. Finally challenges and prospects of SiNW solar cells are presented.

Keywords: silicon nanowires, solar cells, fabrication, optical properties, electrical properties 


\section{Introduction}

World energy consumption refers to the total energy used to support human activities all over the world. Data from BP shows that the dominant energy consumption is oil [1]. However the price of oil fluctuates as the international political and economic situation. Moreover, the emission of carbon dioxide emitted when fossil fuels are burnt aggravates the concentration of carbon in the earth's atmosphere with unpredictable but almost certainly nasty effects that would otherwise accelerate global warming. To curb the emission of carbon dioxide, renewable energy is one of the most promising alternatives. Renewable energy grew more rapidly than any other time in history against the background of energy crisis, which can possibly be explained by an increase in international investment in renewable energy and the demand for green and safe substitution after the accidents at Chernobyl and Fukushima. Solar energy, candidate for replacing traditional fossil fuel energy, shows its advantages on abundance and environmental-friendliness. However, commercial panels are already approaching the limit of energy conversion efficiency [2]. In order to reduce the overall cost per watt of solar cell production, cutting down the materials use is a direct way to decrease cost. Here we review the silicon nanowire solar cell technology because the photovoltaic (PV) market is dominated by silicon-based panels, with a share of $\sim 90 \%$. Fig. 1 (a) plots energy volume harnessed by human beings per dollar and the inset is the price history of silicon PV cells versus annual imported crude oil price for recent decades. Oil crisis have rocked crude oil price by exceeding 100\% since 1973, still drastic fluctuations remain since that collapse (blue curve). Afterwards, oil reservoirs are more inaccessible and need more pricy techniques for extraction so that crude oil supply turned out to be unable to respond to increasing demands. Contrary to high price and dramatic fluctuation of oil price, the price of silicon solar cells drops sharply according to Swanson's law. The price of silicon PV cells is 76 \$ per watt in 1977 while the current price decreases to $0.3 \$$ per watt caused by technology advances and governmentoriented policy support through feed-in tariffs. According to Fig. 1 (a), the harnessed energy of silicon solar cells at the cost of a dollar has surpassed its oil counterpart since 2004. It was anticipated that electricity from PV will be competitive with wholesale electricity costs all across Europe and the energy payback time (EPT) of crystalline silicon modules can be reduced to below 0.5 years by 2020 [3]. Fig. 1 (b) illustrates the carbon intensity of some key electricity generation technologies. The $\mathrm{CO}_{2}$ emission is relatively high when compared with wind and nuclear. Therefore, study on cost-effective silicon solar cell is extremely rewarding for energy crisis and $\mathrm{CO}_{2}$ mitigation. 
(a)
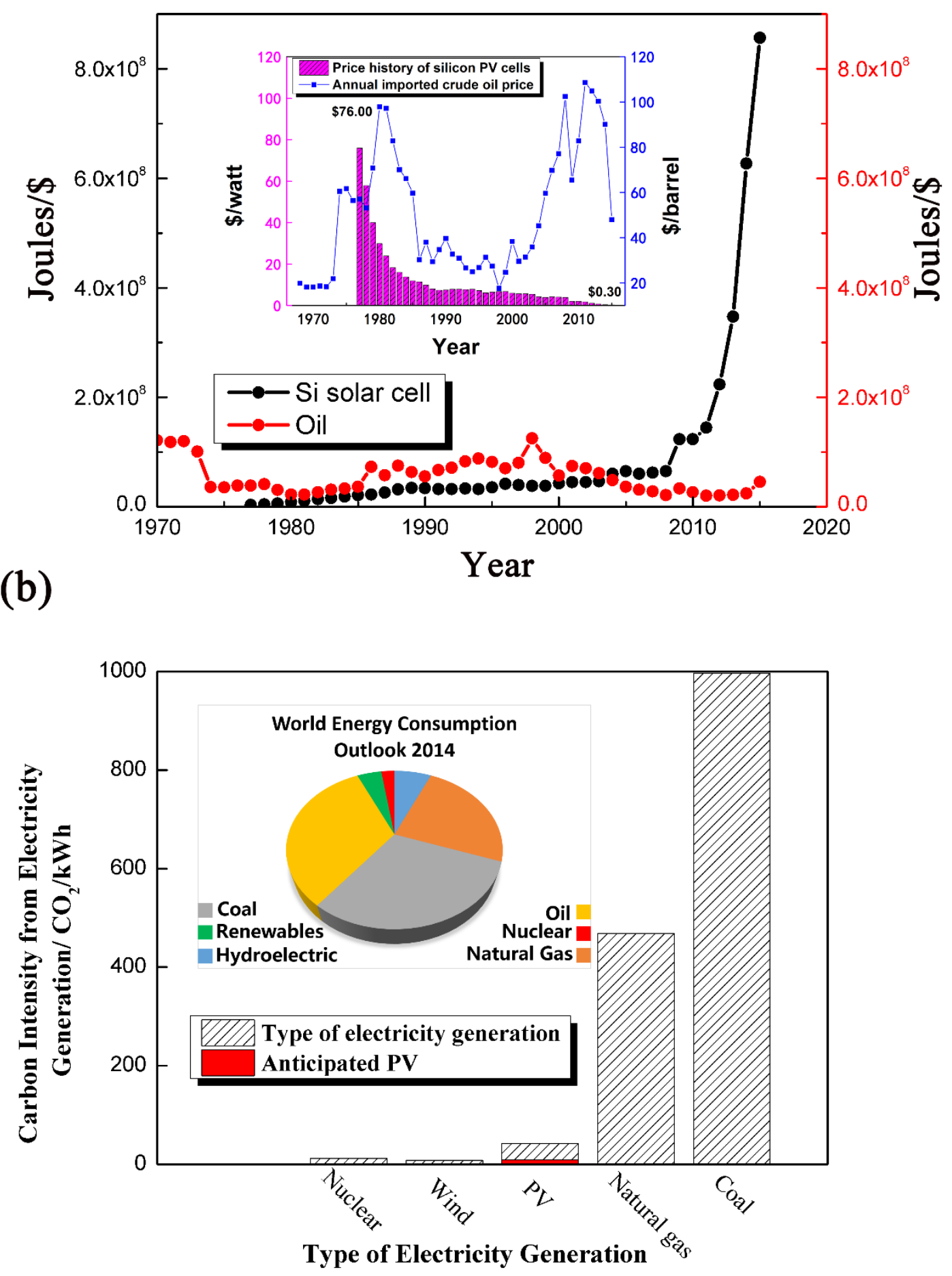

Figure 1 (a) Energy volume of Si solar cells and oil harnessed by human beings per dollar, the 2015 is the predicted value. The inset is price history (dollar per watt) of silicon PV cells (column) and annual imported crude oil price (dollar per barrel, blue dot line), replotted data from Ref. [4, 5]. (b) Carbon intensity of some key electricity generation technologies; the value for PV refers to manufacture in Europe; the red bar is the anticipated value after application of SiNWs solar cells, replotted from Ref. [6]. The inset is world energy consumption 2014 by fuel projection, replotted 
from Ref. [7].

Crystalline silicon materials dominate the photovoltaic (PV) industry with nearly $90 \%$ share in commercial solar cell modules and the Si wafers alone account for $\sim 50 \%$ solar cell cost. As one of the most studied solar energy materials, silicon has a number of advantages, including high abundance (silicon is the second most abundant element on earth), high diffusion lengths of minority carriers, superior mechanical and thermal properties, a suitable energy bandgap ( 1.12 $\mathrm{eV}$ ) matching to the terrestrial solar spectrum, non-toxicity, and mature fabrication techniques. However, despite all the merits above, the indirect band gap and low absorption in solar spectrum of silicon requires a large amount of materials (200 $\mu \mathrm{m}$ thick) to absorb all the incident sunlight with photon energy greater than the Si bandgap energy. Since a thick silicon layer is necessary for absorption, a long diffusion length of minority carriers is then essential to efficiently collect the photogenerated carriers, which requires high quality silicon materials. These requirements lead to a trade-off between the cost and efficiency of silicon PV cells. The metallurgical grade silicon (mg-Si) is relatively cheap with about $\$ 1.75-\$ 2.30 / \mathrm{kg}$ [8], but it has too many impurities to obtain acceptable efficiencies. On the other hand, although solar-grade Si (sg-Si) wafers with higher quality have the minority-carrier diffusion lengths comparable to the long optical absorption depth ( $200 \mathrm{um}$ ), the additional refining steps for upgrading mg-Si to sg-Si give rise to an additional cost of about $\$ 20-\$ 30 / \mathrm{kg}$ [9]. In addition, conversion from mg-Si to sg-Si is highly energyconsuming, covering nearly half of the total energy needed for the final PVs, and thus significantly increases the EPT [10].

Therefore, considerable efforts have been devoted to finding alternative cost-effective PV technology based on silicon and beyond, such as amorphous Si (a-Si), cadmium telluride, and copper indium gallium selenide (CIGS) and organic PV technologies [11-14]. Even though these solar cells provide an increasingly competing performance as sustainable energy sources, they still lack price advantage in comparison with fossil fuels. Accordingly, in the last decade, intensive investigations have been focused on engineering nanomaterials for PV applications due to their unique optical and electrical properties. Novel PV concepts and architectures have been proposed and demonstrated over the years, including hot carrier solar cells [15, 16], multiple-exciton generation [17, 18], intermediate band solar cells [19, 20], and upconversion/downconversion [2126]. Nanostructured materials with different dimensions, such as quantum wells [27, 28], nanorods [29-35], nanowires [36-59], nanotubes [60-65], quantum dots [66-70], and nanocrystals [15, 7173] , play a significant role in the next generation PV concepts. These novel nanostructured designs have achieved great improvements in light absorption and charge separation and collection.

Particularly, one-dimensional semiconductor nanowires have been envisioned as promising alternative building blocks for high efficiency PV cells. In this paper wires with diameter no more than $1 \mu \mathrm{m}$ will be referred to as nanowires. To fulfill the promises of silicon, the most promising and successful PV material so far, nanowire-based PV devices are considered as one of the most viable routes to efficiently convert solar energy into electricity at low cost [49, 74-77]. SiNWs arrays are less sensitive to impurities due to bulk recombination, and thus silicon wafers with lower purity can be used to achieve similar efficiency compared with their planar counterparts, 
leading to the reduction of production cost [78]. The unique geometrical features also are advantageous in full solar spectrum utilization by intrinsically providing broadband optical absorption and superior photogenerated carrier collection. Therefore, SiNWs, requires 100 times less material to achieve the same amount of absorption as in thick crystalline silicon (c-Si) solar cells [79]. Since most $\mathrm{CO}_{2}$ emission during $\mathrm{Si}$ solar cells fabrication comes from $\mathrm{Si}$ wafer preparation, the SiNW solar cells are anticipated to contribute to $\mathrm{CO}_{2}$ mitigation while maintaining efficiency, as the red bar shown in Fig. 1 (b).

Substantial progress has been made in developing SiNW solar cells and the efficiency of SiNW solar cells have increased dramatically in the last few years. Despite all the advantages of SiNWs and the progress made in nanowire based PV cells, the efficiency of SiNW solar cells is far from satisfactory compared to their theoretical value and planar counterparts. A number of technical challenges, such as contamination, surface recombination, and metal contacts, has yet to be addressed for higher efficiency and more cost-effective solar cells. In this paper, to provide a guide to future development in nanowire solar cells, recent progress made on solar cells based on SiNW arrays is reviewed. First, the unique optical properties of nanowires will be discussed. The fabrication methods of SiNW arrays will then be introduced. The discussion includes both bottomup and top-down techniques. Next, we will introduce diversified SiNW solar cells moving towards high performance solar cells. Eventually, challenges and prospects of SiNWs will be discussed.

\section{Optical properties of SiNWs}

\subsection{Influence of nanowire arrangement on light trapping}

Since optical absorption is one of the main factors determining the solar cell efficiency, the optical absorption characteristics need to be investigated to optimize nanowire geometrical parameters, such as periodicity, length, and diameter, for high optical absorption. However, parasitic absorption by substrates and surface defects during sample preparation can make it ambiguous to accurately probe the optical characteristics of nanowires.

To understand the optical properties of dense and sparse SiNWs, Hu et al. simulate the properties of light absorption in ordered SiNW arrays using the transfer matrix method [80]. From this study, much lower reflectance compared to thin films can be obtained from nanowire arrays with a moderate filling ratio. However, good antireflection of the nanowire arrays does not necessarily guarantee desirable absorption unless a lower transmission is achieved at the same time. Unfortunately, high optical transmission is observed in the ordered SiNW arrays for low energy photons and thus there is less overall absorption of the nanowire arrays than their thin film counterparts due to high optical loss in low-frequency regime. Nonetheless, the superior antireflection property of SiNW arrays attracts much attention in the PV community. Later on, excellent light absorption properties have been reported in these SiNWs and many research efforts have been devoted to new designs to improve light absorption and reduce the usage of materials. Using a full wave finite element method, Li et al. indicated the small array periodicity (P) of 100 $\mathrm{nm}$ used in Hu's simulation might possibly account for the high light transmission [81, 82]. As shown in Fig. 2, when the characteristic dimension of nanowires is comparable to incident light 
wavelength $(\lambda \approx \mathrm{P})$, the light scattering can significantly increase the optical path length and result in enhanced light trapping. Nanowire arrays with a large periodicity can contribute positively to decrease light transmission for low energy photons. On the other hand, when the P is larger than the wavelength of incident photons, transmission of photons at the high energy regime becomes notable. As a result, SiNW arrays with periodicity ranging from $300 \mathrm{~nm}$ to $1200 \mathrm{~nm}$ (optimal value $\sim 600 \mathrm{~nm}$ ) with a filling ratio of about 0.2 , or diameter/periodicity $(\mathrm{D} / \mathrm{P})=0.5$, can greatly enhance light trapping in SiNW arrays. Additionally, there also exists a trade-off between the light transmission and reflection of nanowire arrays for different $\mathrm{D} / \mathrm{P}$ ratio, and the optimal value of $\mathrm{D} / \mathrm{P}$ ratio is found to be around 0.8 for optimized light trapping, which serves as a useful guideline for future nanowire solar cell fabrication [81, 83].

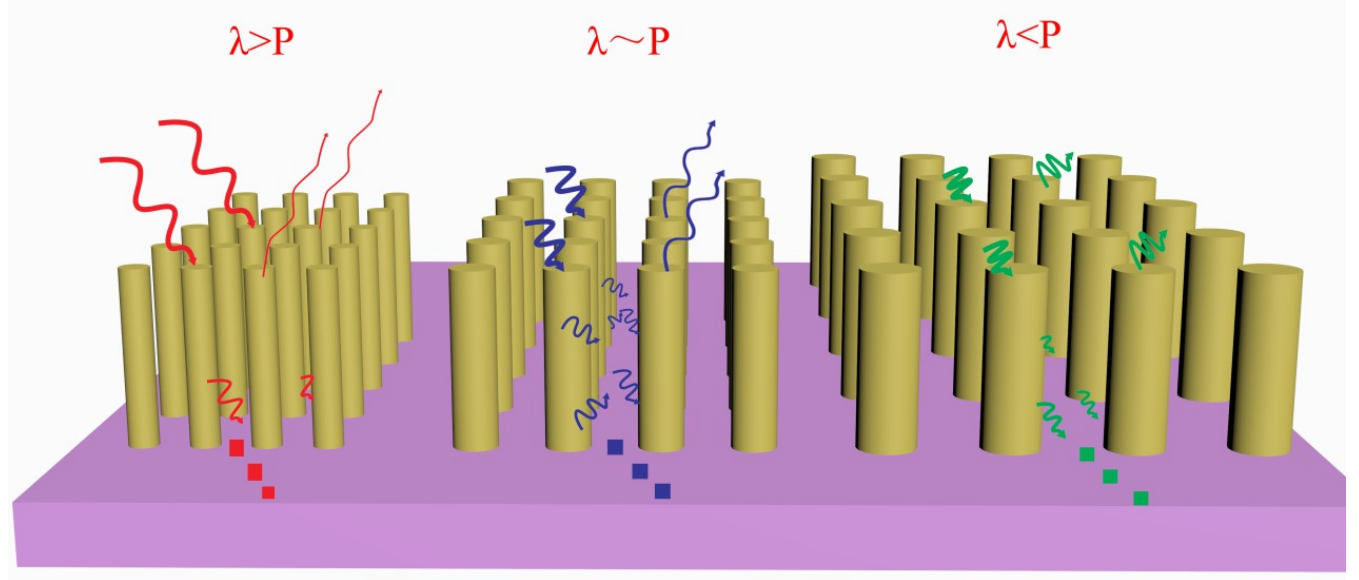

Figure 2 The three representative processes between incident light and nanowire arrays. When the light wavelength $(\lambda)$ is much longer than the array periodicity (P) (left), incident light can easily pass through the nanowire arrays. For the case with $\lambda \sim P$, distinct light scattering lead to elongated optical path length and improved light absorption (middle). When $\lambda<<$, there is strong optical loss due to reflection and transmission.

\subsection{Optical absorption enhancement by resonant modes}

Apart from light trapping based on light scattering, several other mechanisms are put forward to unveil the intrinsic light management of nanowire arrays, such as guided mode resonance [41, 8486]. Li et al. reported that both light scattering and guided resonance can contribute to the enhancement of light absorption in their model [83]. For instance, in SiNW arrays with P and D of 600 and $500 \mathrm{~nm}$, respectively, guided resonance tends to improve optical absorption of nanowire arrays with a wide length ranging from 1000 to $5000 \mathrm{~nm}$, while for wire length under $1000 \mathrm{~nm}$, the light scattering dominates the absorption enhancement. Fig. 3 (a) shows that the ultimate efficiency of the nanowires or light trapping capability increase monotonically with the nanowire length when the wire length is under $1000 \mathrm{~nm}$. However, when the nanowire length is longer (>1000 nm), oscillation appears in the ultimate efficiency curve as a function of wire length. As shown in the inset of Fig. 3 (a), the oscillation is caused by the waveguide mode-induced absorption near the bandgap energy. Using the transfer matrix method, Lin and Povinelli also showed a dramatic optical absorption enhancement in the low-frequency range of the solar 
spectrum when the lattice constant, or period, of the SiNW array increases [87]. The absorption enhancement of nanowire arrays with large lattice constant is attributed to both an increased field concentration and excitation of guided resonance modes in the nanowires. The discrete sharp peaks in the absorption spectrum suggested the presence of guided resonance, resulting in improved light absorption. It should be noted that the overall contribution of guided resonance modes to absorption enhancement may be small because optical absorption still largely relies on light trapping without any guided resonance mode when the length of nanowires goes beyond 5000 nm, as shown in Fig. 3 (a) [86].

However, interesting optical antenna effects in SiNWs have also been analyzed recently [41]. The optical antenna effects are attributed to the large absorption enhancement and broad angular response measured from the SiNWs compared to planar structures. As shown in Fig. 3 (b), one-toone correlation between absorption enhancements and various transverse electric and transverse magnetic leaky mode resonances can be established in a nanowire under normal incident illumination. The presence of leaky mode resonances magnifies the optical absorption by multiple internal reflections and broad band absorption over entire solar spectrum and it can be achieved in nanowires with relatively large $(>100 \mathrm{~nm})$ diameters. As a result of the antenna effects in nanowires, weaker angle-dependence of the optical absorption is observed for nanowire arrays with diameter over $20 \mathrm{~nm}$ compared to planar films. In addition, as the period of the nanowires increases, the angular response further broadens. However, the leaky mode is commonly used to describe the absorption behavior of sparse nanowire arrays [88].

(a)

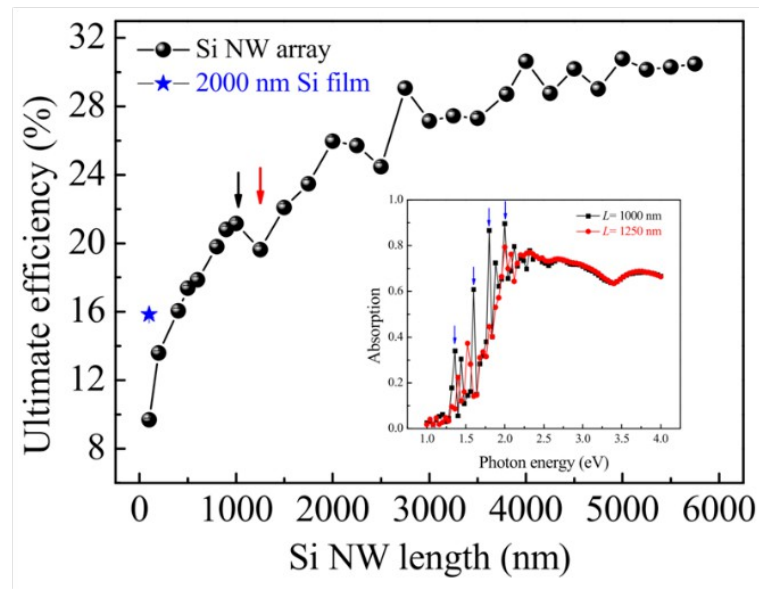

(b)

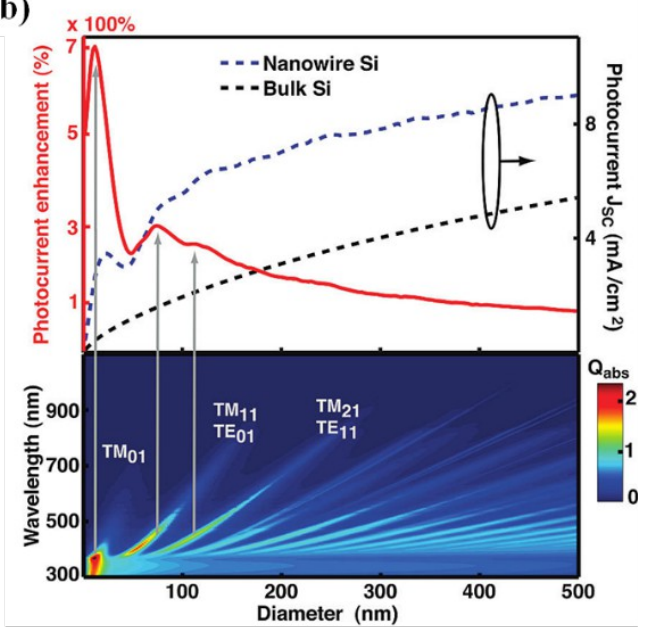

Figure 3 (a) Calculated ultimate efficiency as a function of SiNW length. The SiNW array has periodicity and diameter of 600 and $500 \mathrm{~nm}$, respectively. The inset is the absorption spectra of SiNW arrays with wire length of 1000 and $1250 \mathrm{~nm}$, respectively. (b) (top) Calculated photocurrent density for SiNWs and bulk silicon of comparable thickness. The red solid line plots the photocurrent enhancement of the nanowire. (bottom) Calculated absorption efficiency $\mathrm{Q}_{\mathrm{abs}}$ of SiNWs as functions of the wavelength and diameter a Reproduced with permission from Ref. [83]. Copyright 2012 Institute of Physics. b Reprinted with permission from Ref. [41]. Copyright 2010 American Chemical Society. 
Experimental studies of single SiNW solar cells also revealed that different types of resonant modes other than guided mode resonances can contribute to light absorption in the wires [85, 89]. Kempa et al. showed that substantially larger short circuit current can be obtained from a single SiNW solar cell than that from a bulk Si cell with an equivalent thickness [85]. Moreover, as shown in Fig. 4 (a) and (b), measured external quantum efficiency (EQE) of $p-i-n$ and $p / p-i-n$ exceeds unity for some wavelengths, keeping pace with simulation. The distinct improvement in EQE at certain wavelengths is due to the nanowire resonant modes leading to larger light absorption cross-section than its physical cross-section. Fig. 4 (c) plots different resonant modes simulated by finite difference time domain method (FDTD). The existence of the resonant modes lead to distinct absorption from the entire nanowire but the absorption exponentially decays as a function of thickness in bulk Si. As shown in Fig. 4 (d), the nanowire shows better light harvesting performance than bulk $\mathrm{Si}$, which proves superior capability of nanowire solar cells in reducing material usage while maintaining performance. These resonant modes excited in the nanowire cavities are assigned to wavelength dependent Fabry-Perot, whispering-gallery, and complex, high-order two-dimensional modes [85, 89]. These resonant modes can be further manipulated by changing the nanowire diameter, shape and morphology. For instance, nanowires with rectangular cross-section display enhanced spectral response for low energy photons with respect to a hexagonal nanowire as a result of resonant modes excited in such a high symmetry nanocavity. More importantly, a row of nanowire arrays preserve the similar absorption properties of the single nanowire owing to a diffraction effect of the periodic nanowire array [90]. Recently, it has been shown that these resonant modes are also related to the open circuit voltage $\left(\boldsymbol{V}_{\boldsymbol{c}}\right)$ of nanowire solar cells [91].

Although, the resonant mode exists in SiNW, the difficulty in fabricating uniform nanowire arrays and the negligible guided resonances also make it less attractive in practical engineering of high efficiency nanowire solar cells. Optical antenna effects can be applied to achieve wide-angle absorption enhancement.

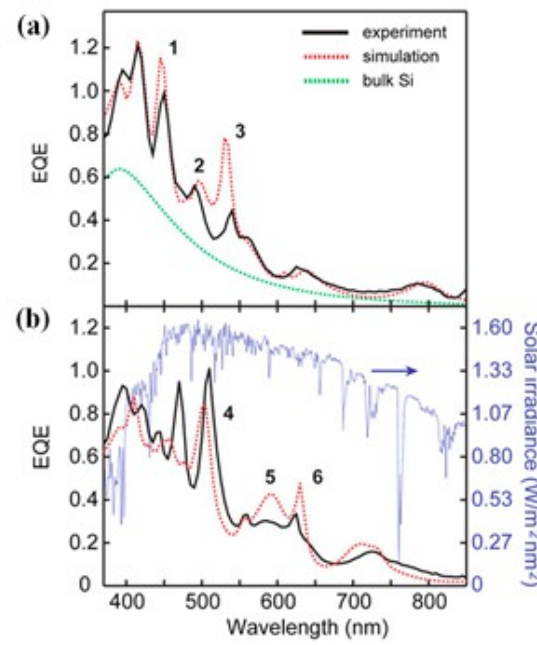

(c)

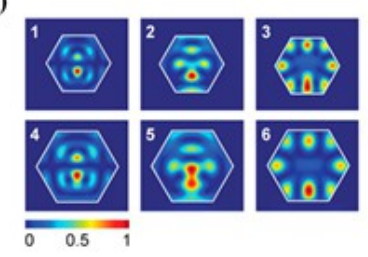

(d)
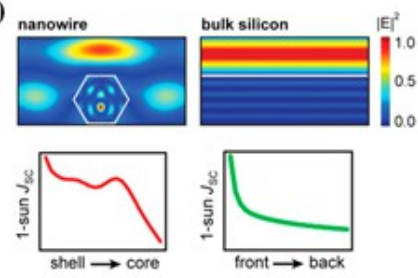

Figure 4 (a) EQE spectra from experimental measurements (black curve) and simulation (dashed red curve) of a $p-i-n$ nanowire. Calculated EQE spectrum from the planar bulk Si of equivalent thickness is plotted for comparison (dashed green curve). (b) Measured EQE spectrum (black curve) of a $p / p-i-n$ nanowire compared to the AM1.5G solar spectrum (dashed blue curve) and 
calculated EQE (dashed red curve). (c) FDTD simulations of resonant mode spatial profiles for the features shown in (a) and (b). (d) Electric field intensity map for plane wave $(\lambda=445 \mathrm{~nm})$ interacting with a nanowire (Left) and bulk Si (Right) and plots of total short circuit current density as a function of position inside nano- and bulk materials, respectively. Reprinted with permission from Ref. [85]. Copyright 2012 National Academy of Sciences, USA.

\subsection{Tapered nanowires for broadband and omni- directional absorption}

The geometry of the nanowires also plays a critical role in light absorption and should be taken into consideration during design of SiNW solar cells. It has been shown that near perfect antireflection property can be achieved over a wide range of wavelengths and incident angles by forming a graded refractive index profile between the air and the surface [92]. In addition to increased absorption under angle of incidence, the tapered nanowires are compatible with large molecule in hybrid SiNW solar cells [93].

One effective way to generate the graded refractive index is to fabricate tapered nanostructures. In addition, using group theory, Han and Chen also showed that broadband and omni-directional absorption exceeding the Lambertian limit can be possibly realized [94]. By breaking the symmetry of the sub-wavelegnth nanostructures and hence reducing mirror reflection, Lambertian limit can be nearly achieved in tapered two-dimensional gratings at normal incidence. Replacing the tapered two-dimensional gratings with array-based asymmetric tapered tips exhibits absorptance approaching the Lambertian limit over a wide angle of incidence. As shown in Fig. 5 (a), the surface texture on Si surface can significantly improve the ultimate efficiency compared with the unstructured surface. Rod arrays with nonsymmetric tapered tips show the highest ultimate efficiency than other structures and exceed the Lambertian limit over a large range of thickness. Huang et al. reported broadband and near omini-directional reflection of SiNWs with tapered tips on an entire 6-inch silicon wafer [92]. In this work, needle-tapered SiNWs are fabricated via an electron cyclotron resonance plasma process, which can create high aspect ratio nanotips/nanowires in a large scale without using any additional mask or lithography step [95]. Such tapered Si nanostructures lead to excellent anti-reflection spectrum not only in the visible region but also in far infrared regime. Besides, superior anti-reflection performance $(<1 \%)$ is measured in a wide range of incident angles from $5^{\circ}$ to $60^{\circ}$ in Huang's work. This method has also been adopted by using ordered SiNW/nanocone arrays [96]. Compared with planar thin film and nanowires with uniform diameters, the cone-shaped amorphous Si nanostructures show improved absorption because of a gradual increase of the effective refractive index from the Si surface to air. As shown in Fig. 5 (b), over 90\% of quasi-omni-directional absorption is observed from the $\mathrm{Si}$ nanocones as incident angles increase to $60^{\circ}$. Fig. 5 (c)-(e) show the arrays of silica nanoparticles, tapered nanowires, and un-tapered nanowires. Although un-tapered wires still show distinct improvement in absorption at a wide range of angles, tapered nanowires further improve the absorption by $10 \%$ when incident angle increases to $90^{\circ}$. Dual-diameter Ge nanowires are also presented outstanding absorption of $\sim 99 \%$ of the incident light over the entire visible regime [97]. While the small diameter tip fabricated on the top of the nanowire arrays minimizes the reflection, the base of the wires with a large diameter prevents optical loss through transmission. Later, Hua et al. showed that multi-diameter nanowires were capable of achieving broadband light absorption 
[98]. Although Ge nanowires were merely demonstrated in Fan's work, this method can also be applied on SiNWs for maximizing absorption of photons over a broad spectral band.
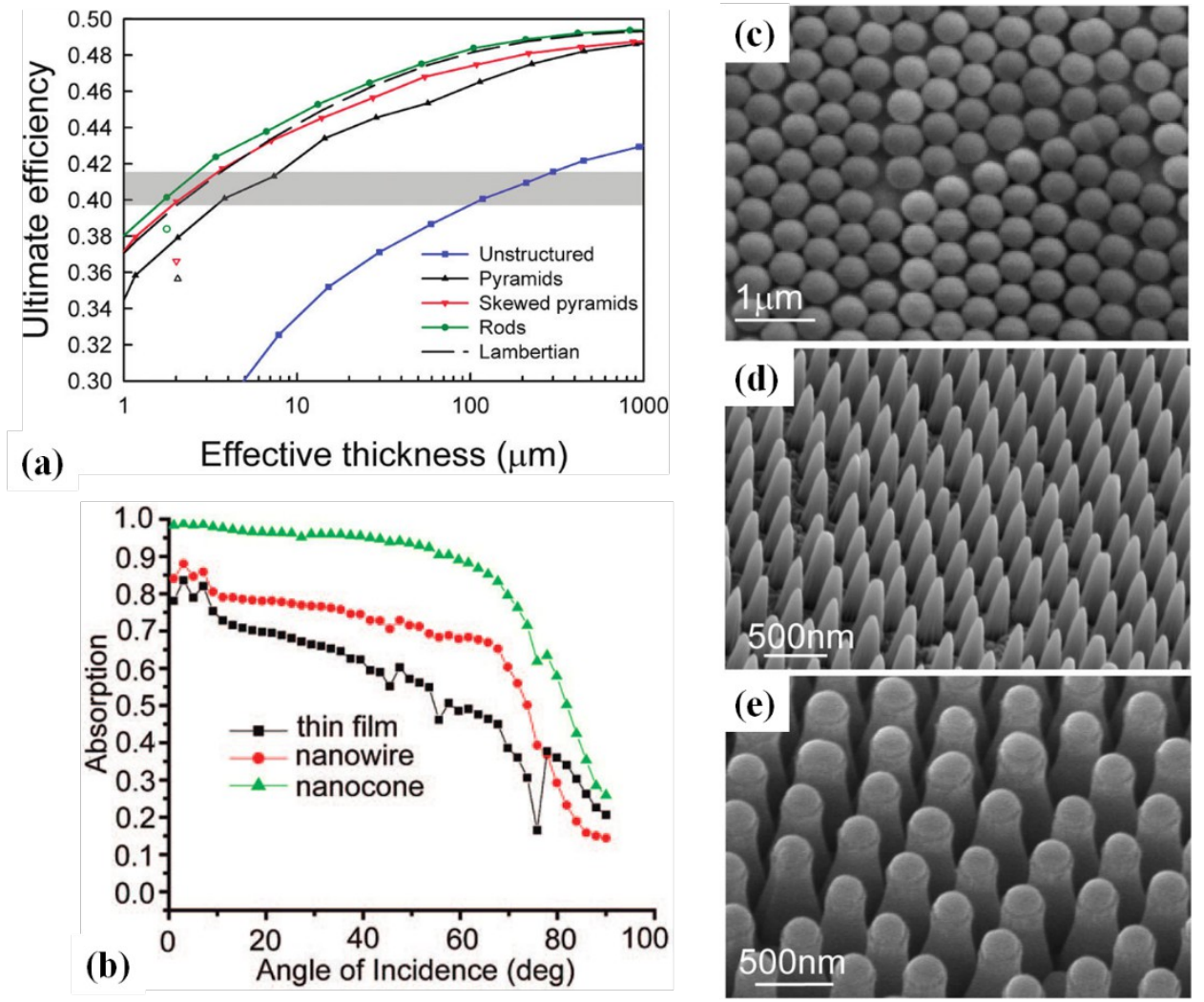

Figure 5 (a) Ultimate efficiency of various structures as a function of effective thickness. (b) Measured absorption at wavelength of $488 \mathrm{~nm}$ for a-Si:H thin film, un-tapered nanowire arrays, and tapered nanowire arrays at different angles of incidence. (c-e) SEM images of arrayed silica nanoparticles, tapered nanowires, and un-tapered nanowires. a reprinted with permission from Ref. [94]. Copyright 2010 American Chemical Society. b-e reprinted with permission from Ref. [96]. Copyright 2009 American Chemical Society.

\subsection{Order versus disorder}

Most studies, particularly theoretical work, have focused on ordered nanowire arrays. In contrast with low reflectivity of ordered vertical nanowires, disordered nanowires with random orientations generally have significant diffuse reflectivity arising from multiple scattering and the reflectance, reaching as high as $60 \%$ [38, 99-101]. Analogous to the random textured surface for antireflection, nanowire arrays with random length induce multiple optical scattering and field localization, which leads to the enhanced absorption and anti-reflection properties. Bao and Ruan numerically showed that vertically aligned nanowire arrays with random position or diameter can also achieve similar low reflectance and high absorption as the ordered array with uniform diameter [38]. The random diameters of the nanowire arrays can lead to different broadened resonant frequencies which give rise to absorption enhancement. While the absorption at the original off-resonance frequency is enhanced, the original resonance frequency maintains high, caused by multiple scattering induced by the random arrangement or diameter of nanowires. Sturmberg et al. revisited disordered nanowire arrays with non-uniform diameters using a modebased numerical method and semi-analytic approach [101]. Fig. 6 (a) shows that radial disorder 
can increase the ultimate efficiency independent of the lattice constant of nanowire arrays. Any radial variation via moving off the diagonal line in Fig. 6 (a) for any lattice spacing can improve the ultimate efficiency. Meanwhile, the superposed absorption of the sublattices with different nanowire diameters can curb the issue of ordered nanowire arrays with a limited wavelength range of enhanced photon absorption. As shown in Fig. 6 (b), the absorption spectrum of a designed array consisting of four sublattices and the absorption spectra of arrays with only diameter differs from each other. The radial disorder design process leads to high absorption over a broadband spectrum. As each sublattice barely affects the modes of each other, the superposed absorption spectra of sublattices with different diameters can lead to a broadband enhancement of photon absorption. By using the radial disordered SiNW arrays containing only 30\% silicon, a significant improvement in ultimate efficiency of $23.75 \%$ can be achieved, compared to $17.6 \%$ of regular nanowire array and $13.8 \%$ of thin film with equal thickness.
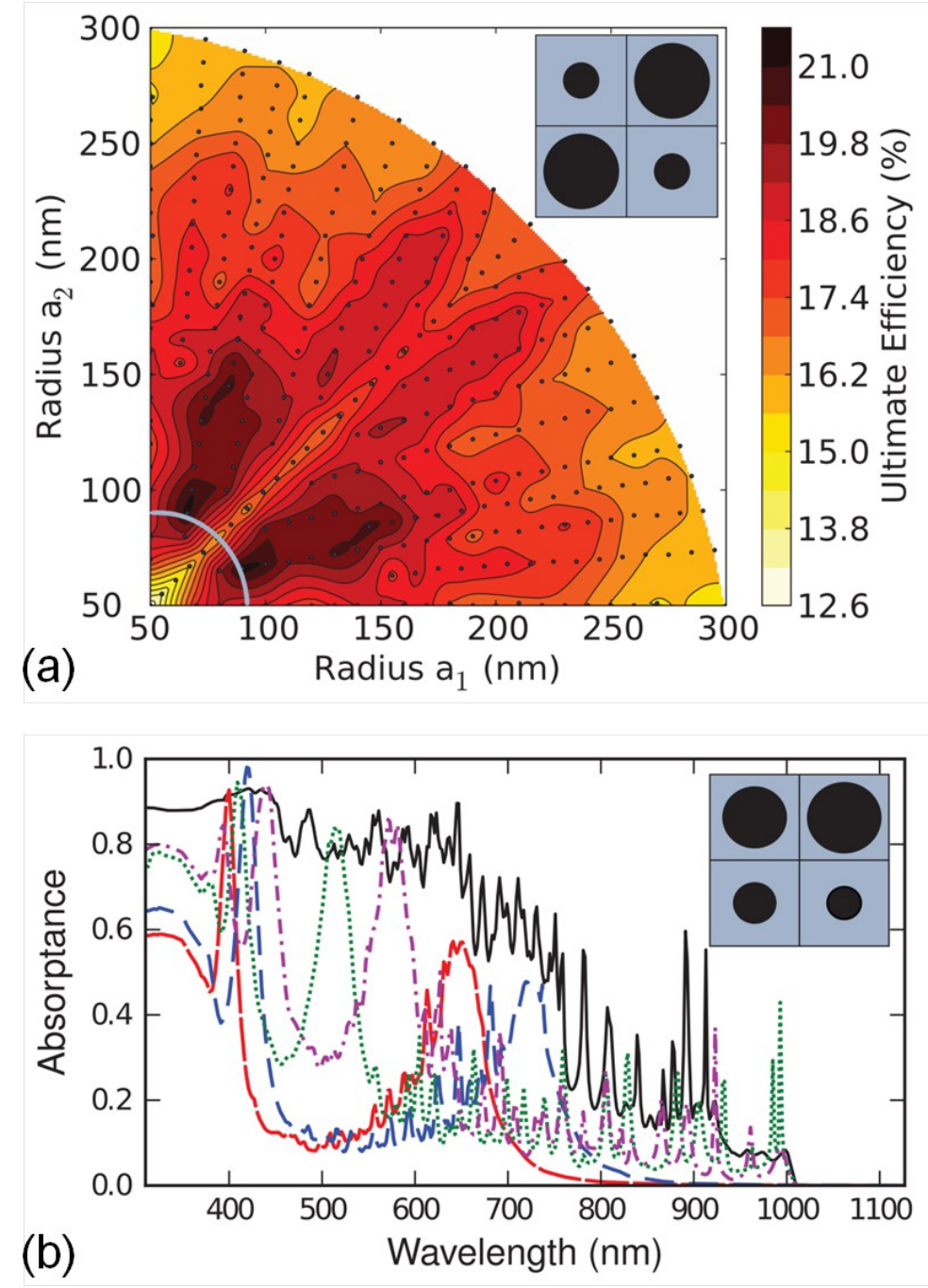

Figure 6 (a) Ultimate efficiency for SiNWs arrays consists of two sublattices with different radius. The inset illustrates a typical unit cell. (b) Absorption spectra of the nanowire array with four sublattices (black curve). The unit cell is illustrated in the inset. Color broken curves are the absorption spectra of each individual sublattice. Reproduced with permission from Ref. [101]. Copyright 2012 American Institute of Physics. 
Lin and Povinelli optimized aperiodic nanowires using random walk algorithm resulting in an over $100 \%$ enhancement factor in photovoltaic efficiency compared to the periodic counterparts [102]. In the periodic array, absorption of high energy photons is higher than thin film because of reduced reflection from the top surface, but calculated absorptance for early band edge photons dwarfs due to the significant transmission loss. By using aperiodic nanowires, similar excellent anti-reflection can be achieved for high energy photons and absorption at long wavelength regime is not degraded. On the contrary, multiple peaks present in the absorptance spectra at lower energies resulting in absorption enhancement. The calculated power loss rate of the optimal aperiodic nanowire array reveals a few localized regions with strongly enhanced absorption. Similar to periodic nanowire array with a larger lattice constant, the improved efficiency of the nanowire solar cells can be explained by enhancement in field concentration and the excitation of localized resonance modes [102]. By using a larger lattice constant (500 nm instead of $100 \mathrm{~nm}$ in Lin and Povinelli's work), Du et al. also reported improved absorption performance in random nanowire arrays [48]. The absorption, transmittance, reflectance, and electric field intensity distribution are compared by using FDTD method, which demonstrates that the overall improvement in absorption of aperiodic nanowire arrays is caused by better anti-reflection, additional resonant modes from the disorder, and broadening of existing resonances. Interestingly, Wei Wang et al. imitate the rainforest configuration in nanostructure-based solar cell design [103]. Novel multilayer nanorainforest solar cells based on p-Si nanopillar array/n-CdS nanoparticles/n$\mathrm{ZnO}$ nanowire array heterostructures were fabricated via a highly accessible, reproducible and controllable fabrication process, meaning that this wafer-scale, low-cost and easily controlled processing has potential on nanostructure PV devices. By choosing materials with appropriate bandgaps, an enhanced efficiency was obtained due to the wide- covered nanostructure for wide range of the solar spectrum absorption, a kind of similar to tandem solar cells.

Random lengths can be realized on purpose to further suppress the reflection as Silke L. Diedenhofen demonstrated [104]. By controlling the NW length distribution, any local filling ratio $f$ (defined as $\pi d^{2} / 4 a^{2}$, where $d$ is the nanowire diameter and $a$ is the lattice constant) profiles can be realized. In addition to reflection suppression, it can be expected that the random length can induce field localization [105]. Their simulation shows that random positioned nanowires with an average lengths $1.5 \mu \mathrm{m}$ ranging from 1 to $2 \mu \mathrm{m}$ are advantageous on suppressing reflectance. When $f=0.2$ the reflectance is already quite small, the reduced reflection is not evident. The enhanced absorption is mainly attributed to the optical scattering. When $f=0.5$, the ordered NW array has a much larger reflectance while the random one can minimize the reflectance.

However, the random structures are mainly based on optical absorption without taking into consideration of charge separation and collection. For practical nanowire solar cell design, the ideal nanowire array design for photon absorption may not necessarily optimal for nanowire solar cells. For instance, disordered nanowires can possess unequal electrical characteristics, which pose a limitation to the overall efficiency of the nanowire solar cells. Moreover, ordered and uniform SiNW arrays is highly desired to form high quality p-n junction and electrical contacts [86]. Nevertheless, the general dependence of the optical properties of SiNW arrays on its structural parameters is an important guideline for tailoring high efficiency nanowire solar cells at low cost. 


\subsection{Tilted SiNWs versus vertically aligned SiNWs}

Extensive simulations and experiments are conducted to give a better understanding of the vertically aligned SiNW arrays. In contrast, the slanting SiNWs attract attentions on their special characteristics as well. Simulation works have compared the light harvesting ability between the slanting and vertically aligned SiNW structure [106-108]. The slanting SiNWs architecture demonstrates a strong suppression of reflectance and enhanced light trapping ability with highest ultimate efficiency of $33.45 \%$, better than its freestanding counterparts (28.36\%) [106]. With the same amount of material, tilted SiNWs present improved performance compared to vertical nanowires arrays over a broad range of tilt angles (from $38^{\circ}$ to $72^{\circ}$ ) because of excitation of additional modes besides the $\mathrm{HE}_{1 \mathrm{~m}}$ modes [107]. Specifically, the optimum tilt angle of $53^{\circ}$ has an improvement of $8.6 \%$ over that of the vertical nanowire arrays and $80.4 \%$ of the ideal double-pass thin film. For a typical vertical axial p-i-n NWs, if the nanowires are tilted from the substrate, much of the incident light can be directly absorbed by the active region without passing through the $\mathrm{p}(\mathrm{n})$ region, and thus greatly enhance the absorption of the active region and raise the efficiency. Inclined SiNWs with growth direction of $\langle 110>$ and $\langle 112>$ on $<111>$ are simulated [108]. They showed better efficiency enhancement (4\% and 3.87\% respectively) than the vertical SiNWs solar cells (3.41\%). The different optical properties of inclined SiNWs and vertically standing SiNWs is mainly due to the reduced mirror symmetries from four for the vertical SiNWs to only one for the slanting SiNWs [109].

By utilizing the anisotropic etching and controlling the etching solution concentration, experiments are demonstrated to fabricate tilted nanowires [110-112]. In fact, the SiNWs produced from Si (100) and (111) wafer are vertical to surface in chemical etching process. However, SiNWs from Si (110) and (113) substrates are arranged slantly, as shown in Fig. 7 [110]. Contrary to the Si (100) and (111) substrates with SiNWs along the $<100>$ and $<111>$ directions, respectively, the (110) and (311) wafers breed SiNWs both along $<100>$ direction, meaning that the wire axis is perpendicular to the [100] lattice plane.
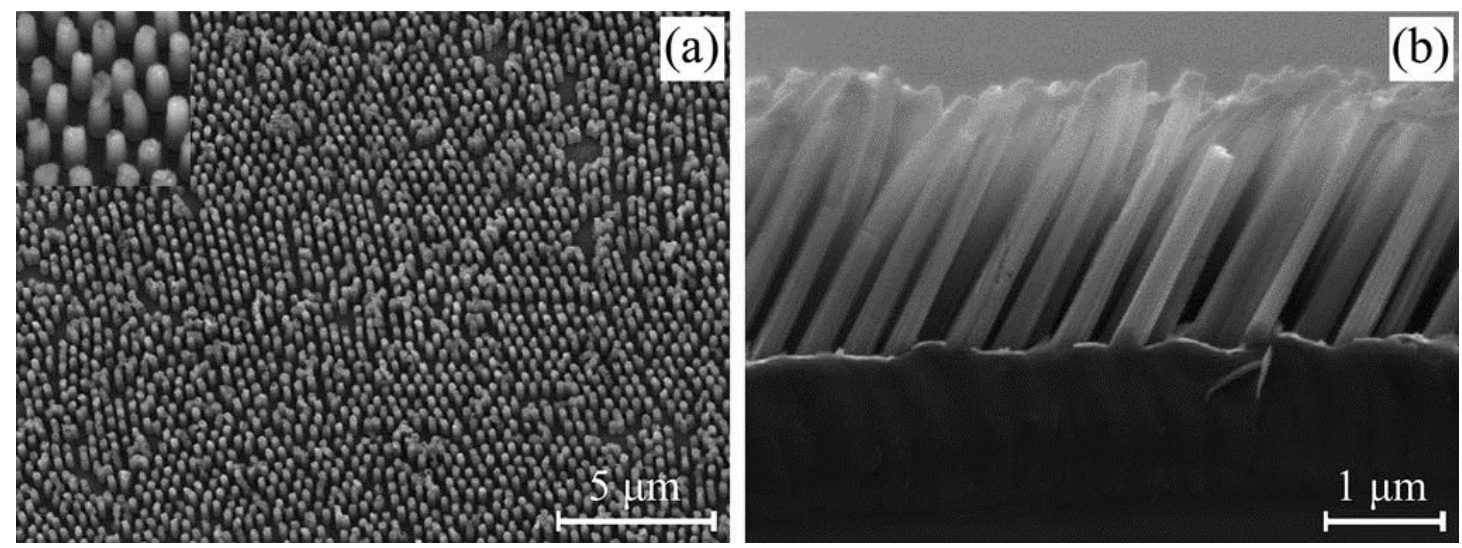

Figure 7 (a) Top view of large-area ordered SiNWs produced on n-type Si (113) substrate. (b) Typical cross-sectional SEM image showing tilted aligned SiNWs produced on n-type Si (113) substrate. Reprinted from Ref. [110] with the permission of AIP Publishing.

However, obtaining the tilted SiNWs with arbitrary angle is a tough task under solution etching. 
The abovementioned experiment have limits on precisely wide angle control and nanowire shape maintenance during etching process. Dry etching methods maybe applicable via change the slant angle between substrates and etching ion beam. For example, a silica nanospheres assisted ion bombarding (SAIB) method to fabricate patterned oblique NW arrays of polyimide (Kapton) film was reported [113].

To summarize this section, table 1 concludes the parameters for optical enhancement of SiNWs including nanowire length, diameter, lattice constant, ordered/disordered, shape and orientation. The listed references are not exhaustive; only a few representative references are cited in the table.

Table 1 Typical consideration for optical enhancement of SiNWs.

\begin{tabular}{|c|c|c|c|}
\hline Parameter & Dependence & Representative Ref. & Notes \\
\hline Length & $\begin{array}{l}\text { Linear length- } \\
\text { dependent } \\
\text { enhancement with } \\
\text { absorption }\end{array}$ & {$[114,115]$} & $\geq 1 \mu \mathrm{m}$ \\
\hline Diameter & $\begin{array}{l}\text { Absorption increases } \\
\text { with increasing } \\
\text { diameter }\end{array}$ & [116] & \\
\hline Lattice constant & $\begin{array}{l}\text { Absorption increases } \\
\text { with increasing lattice } \\
\text { constant }\end{array}$ & [87] & \\
\hline Ordered/disordered & $\begin{array}{lr}\text { Disordered } & \text { nanowire } \\
\text { preferred } & \text { for } \\
\text { minimizing } & \\
\text { reflectance } & \end{array}$ & [105] & $\begin{array}{l}\text { Deliberate fabrication } \\
\text { of ordered nanowires } \\
\text { are unnecessary }\end{array}$ \\
\hline Shape & $\begin{array}{l}\text { Nanocone } \\
\text { Nanotip } \\
\text { Dual-diameter }\end{array}$ & $\begin{array}{l}{[97]} \\
{[96]} \\
{[92]}\end{array}$ & \\
\hline Orientation & $\begin{array}{l}<111> \\
<110> \\
<112> \\
<100>\end{array}$ & $\begin{array}{l}{[45]} \\
{[117]} \\
{[118]} \\
{[119]} \\
\end{array}$ & $\begin{array}{l}\text { Diameter }>20 \mathrm{~nm} \\
\text { Diameter }<20 \mathrm{~nm} \\
\text { Diameter }<20 \mathrm{~nm} \\
\text { Diameter unsure } \\
\end{array}$ \\
\hline
\end{tabular}

\section{Growth Mechanisms of SiNWs arrays}

\subsection{Vapor-liquid-solid (VLS) growth}

Over the past decades, techniques on nanofabrication have been developed driven by the exotic properties of nanomaterials as well as demands for devices with higher performance. SiNWs have been fabricated by various methods, consisting of both top-down and bottom-up approaches. The versatile technologies, including vapor-liquid-solid (VLS) growth, lithography, electrochemical etching, and metal-assisted chemical (MAC) etching, are capable of making controllable 
fabrication of SiNWs for acquiring efficient solar cells. In this section, the major SiNW fabrication method will be reviewed. Both top-down and bottom-up methods will also be introduced.

The growth of one-dimensional materials using VLS mechanism have been under intensive research in the last twenty years. The growth of SiNWs by VLS mechanism and its analogues have been comprehensively reviewed by Schmidt et al.[45, 59, 118, 120-122]. Here, representative studies, which afford greater control and possibilities in solar cell design, are briefly presented. Going back to the 1960s, the famous VLS growth method of SiNWs was already reported by Wagner and Ellis [120]. In this paper, they found some metal impurities, which remain as droplets and act as catalysts at the tip of the wires during growth, are an essential prerequisite for assembly of one-dimensional silicon wires. The VLS growth of SiNWs, or nanowires made from other materials, involves a vapor phase precursor and a liquid phase catalyst, from which unidirectional nucleation of solid materials proceeds in the form of wires. Recent investigations revealed that the VLS growth mechanism of SiNWs includes mainly three different stages: alloying, nucleation, and wire growth. Fig. 8 (a) illustrates typical processes of the VLS mechanism, using SiNW growth as an example. First, Au nanoparticles are formed on crystalline substrates. After exposing to the vapor phase precursor, e.g. silane $\left(\mathrm{SiH}_{4}\right)$, the alloyed droplets are supplied with silicon. The precursor molecules decompose into gas phase species and the reactive element, silicon. Ross and coworkers used an ultra-high-vacuum transmission electron microscope to confirm the Au-Si alloying process of VLS growth mechanism in real time [123-125]. Initially, the silicon dissociates from precursor wrapped an Au nanoparticle with diameter of $\sim 35 \mathrm{~nm}$ to form a liquid Au-Si eutectic shell. A phase transition from two-phase $\mathrm{Au} / \mathrm{Au}-\mathrm{Si}$ towards singlephase $\mathrm{Au}-\mathrm{Si}$ is observed before the diameter of the Au nanoparticle reaches about $15 \mathrm{~nm}$, at which point, the domain of Au shrinks very quickly and vanishes. A continuous supply of the silicon from the vapor phase precursor supersaturates the droplets with silicon and any additional silicon incorporation results in nucleation of solid silicon at the silicon/droplet interface. They also showed that solid silicon nucleated rapidly at the edge of the Au-Si droplet, indicating that the nucleation is heterogeneous. Nucleation barrier is normally presented to form new crystalline $\mathrm{Si}$ phase from the Au-Si droplets and thus, the Au-Si droplets are supersaturated with Si. The nucleation of silicon is unable to initialize until the barrier is overcome by increasing supersaturation of the Au-Si droplets with more silicon, which is temperature dependent. The continuation of this process leads to droplet catalyzed assembly of SiNWs. In traditional VLS growth model, the catalyst size is assumed to be unchanged during growth and the diameter of nanowires simply follows the catalyst size. However, Hannon et al. found metal loss during the Au-catalyzed SiNW growth using an ultra-high vacuum electron microscope [126]. The nanowire diameter can be affected by the Ostwald ripening of catalyst droplets during growth. In addition, the atoms of the catalyst droplets can be incorporated into the nanowires as well as diffuse away from the nanowires. To solve these issues, Kodambaka et al. proposed to decrease the catalyst loss by adding oxygen to the precursor gas, which forms a partly oxidized nanowire surface and keeps the catalyst material from diffusion away [127]. As shown in Fig. 8 (b), a rapid decrease of the catalyst size is observed until oxygen is introduced around 7230 s. On the contrary, Fig. 8 (c) shows the catalyst disappears rapidly after oxygen is removed from the growth around $17000 \mathrm{~s}$. A patterned oxide layer is also found beneficial to prevent the migration of the catalyst in the early 
stages of wire growth [121]. By using the patterned oxide layer, regular array of Si microwires with uniform diameters can be grown by VLS mechanism, as shown in Fig. 8 (d). However, the catalyst incorporation into the nanowire is unable to be avoided [128], which normally results in poor electronic properties, particularly for Au catalyst. This can be partially solved to some extent by using catalysts like $\mathrm{Al}$, which is a p-type dopant and thus does not introduce deep level defects $[129,130]$.
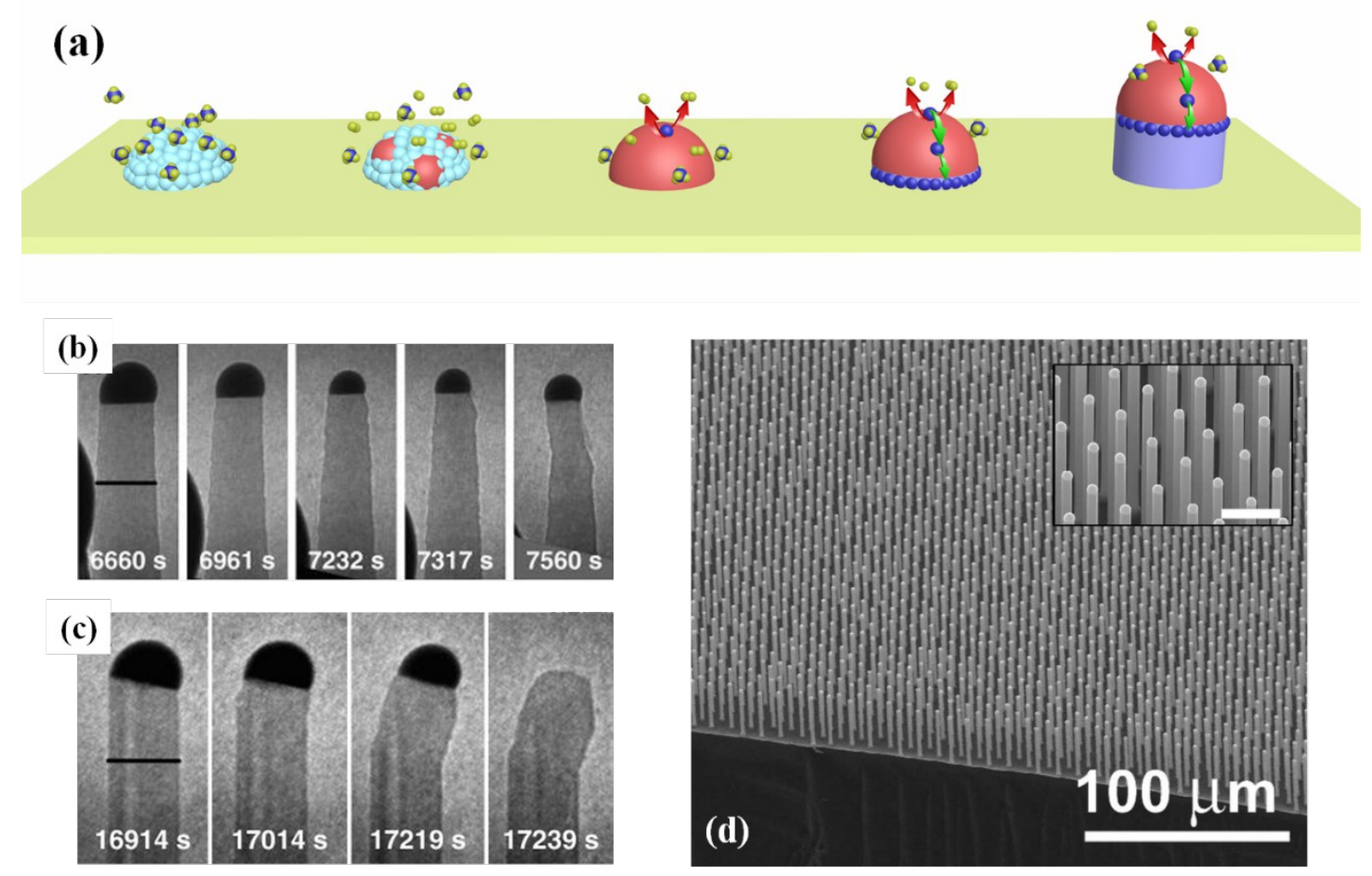

Figure 8 (a) Schematics of the VLS growth mechanism of SiNWs. (b) TEM images of a nanowire grown with $4.5 \times 10^{-6}$ Torr disilane with $5 \times 10^{-7}$ Torr oxygen around $7230 \mathrm{~s}$. (c) TEM images of a nanowire grown with $1 \times 10^{-6}$ Torr disilane and $2 \times 10^{-7}$ Torr oxygen until $\mathrm{t}=\sim 17000 \mathrm{~s}$ when the oxygen is switched off but maintain the disilane pressure. (d) SEM image of a Cu-catalyzed Si wire array grown out from a patterned $\mathrm{SiO}_{2}$ mask. b-c reprinted with permission from Ref. [127]. Copyright 2006 American Chemical Society. d reprinted from Ref. [121] with the permission of AIP Publishing.

The VLS nanowire growth methods have been demonstrated by other techniques, such as chemical vapor deposition (CVD) [118, 126, 131, 132], molecular beam epitaxy (MBE) [50, 133135], and laser ablation [136-142]. Alternatively, vapor-solid-solid [143-145], solution-liquid-solid[146-148], supercritical fluid-liquid-solid [58, 117, 149], supercritical fluid-solid-solid [73], gold implanted silicon substrates VLS method [150], and oxide assisted growth mechanisms [142, 151-153] have been developed. They are the common analogues of VLS mechanism, which rely on a seed particle or catalyst to promote the unidirectional growth of nanowires. Due to the nature of these mechanisms, the choice of precursors and catalysts plays a significant role in the nanowire synthesis [36, 121, 122, 154-157]. Especially, the oxide assisted growth mechanism, an approach to grow SiNWs without the presence of metal catalysts, is favorable for high performance device without metal contamination. This mechanism from thermal evaporation generates nanowire directly from the vapor phase in the absence of a metal 
catalyst, and this process is often called vapor-solid (VS) growth [158]. According to S. Noor Mohammad's discussion, this is actually a vapor-quasisolid-solid (vapor-quasiliquid-solid) mechanism [159].

Using these methods, researchers can readily obtain SiNWs with desired diameter, density, and length through delicate control of growth parameters and preparation of catalyst [160-162]. In addition, growth rates and in situ doping can been tuned precisely. However, experimental and theoretical reports show contradicted results on the rate-limiting step for nanowire growth. Both incorporation-limited and crystallization-limited nanowire growth mechanisms have been reported [163]. For instance, diameter-dependent (crystallization-limited) and -independent (incorporationlimited) growth rate is reported for the VLS growth of SiNW [164-166]. Recently, Pinion et al. identify that both crystallization-limited and incorporation-limited regimes existed during VLS NW growth as schematically shown in Fig. 9 (a) and (b) [163]. By extracting the experimental growth rates under a series of growth conditions using a so-called "ENGRAVE" method, a "phase" diagram of the rate-limited regimes is created. As shown in Fig. 9 (c), incorporationlimited regime (red region) is observed at low temperatures and precursor partial pressures while crystallization-limited regime (green region) at higher temperatures and pressures. The understanding of the microscopic and physical processes of nanowire growth is essential to enable controlled growth of SiNWs for high efficiency solar cells.
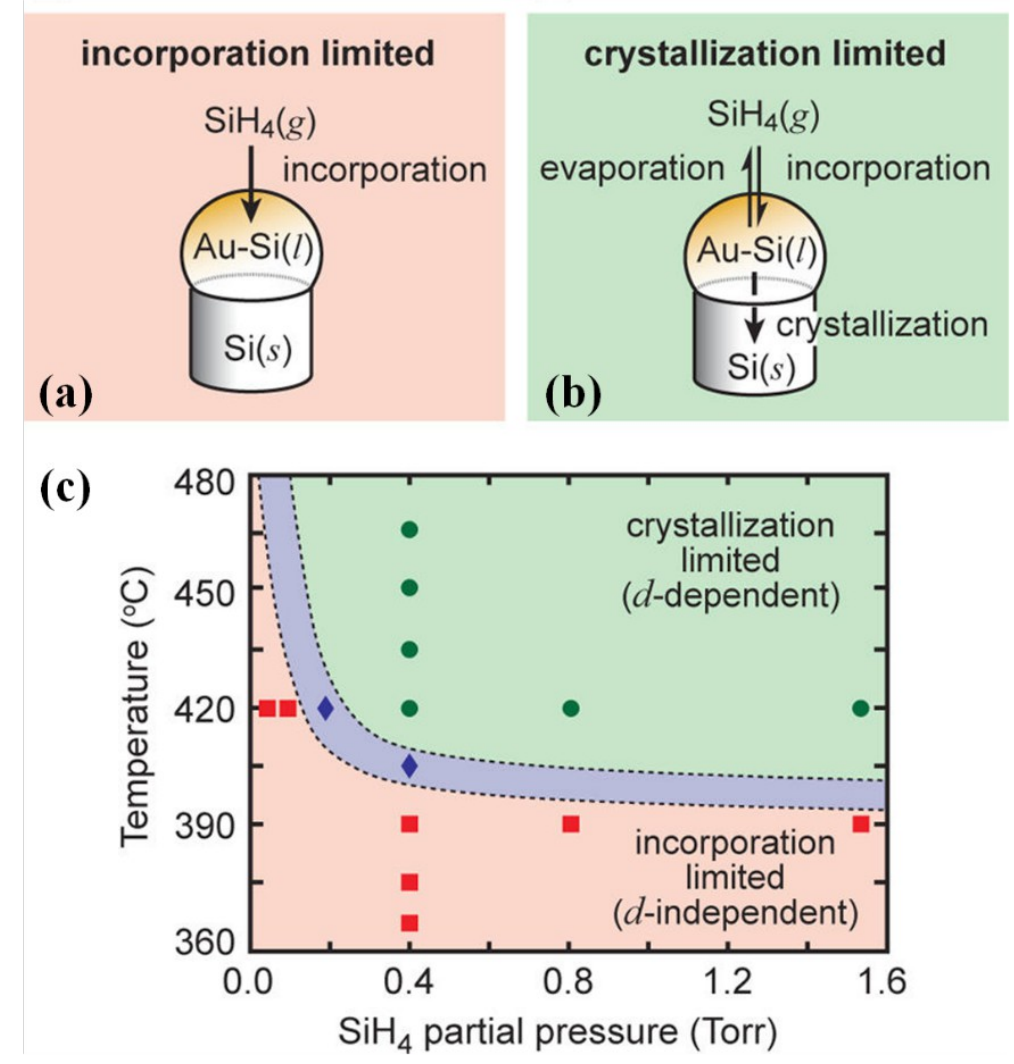

Figure 9 Illustrations of the rate-determining kinetic processes for (a) the incorporation-limited (diameter-independent) regime and (b) the crystallization-limited (diameter-dependent) regime. (c) "Phase" diagram depicting regions of different rate-determining kinetic processes. Reprinted with permission from Ref. [163]. Copyright 2014 American Chemical Society. 
As discussed earlier, the nanowire growth direction and surface arrangement are also one of the critical factors to determine the optical properties of SiNWs arrays. According to thermodynamic theory, nanowires tend to choose the direction that minimizes the total free energy of the system, which includes both the solid/liquid interfacial energy and surface energy [118]. Therefore, precise control of the position and orientation of nanowire arrays is quite challenging due to the growth dynamics can be affected by many factors such as temperature and pressure. Template is an effective means for growing ordered SiNWs. By using the template, the SiNWs can be grown with good control in diameter and position. Zhang et al. reported growth of vertically aligned SiNW arrays by using anodized alumina templates [167]. In this method, the Au catalysts are deposited in the nanopores of the alumina templates by electrodeposition to guide nanowire growth in VLS mode. Later, Bogart et al. prepared nanoporous alumina membranes with different pore diameters which are controlled by the anodization voltage [40]. The diameter of the nanowires grown via the VLS mechanism dovetail with the alumina pore size. Well-ordered SiNWs with good uniformity have been achieved by further optimizing anodic aluminum oxide (AAO) templates and catalyst deposition [54, 168, 169]. For example, perfectly organized alumina nanopore arrays are fabricated by anodization etching combined with nanoimprint lithography. Fig. 10 (a) and (b) shows the arrays of alumina nanopore arrays obtained with a simple anodization and with double anodization and widening and opening of the pores. After the alumina template with desired pore size is formed on the Si substrate via a series of steps of anodization and imprint, ordered nanowires grown via VLS mode can be obtained, as shown in Fig. 10 (c). Instead of using a template, Gao et al. selectively deposit Au catalysts for the growth of aligned SiNW arrays by using galvanic displacement processes [51]. Moreover, the size of the nanowires can be well controlled by the water-in-oil microemulsions used in the galvanic displacement process. Fuhrmann et al. also demonstrated vertically aligned SiNW arrays using nanosphere lithography to create ordered Au catalyst template [50]. Fig. 10 (d-f) shows a monolayer of nanospheres can be used to form ordered Au catalyst array, which further assisted growth of SiNW arrays in the VLS growth mode. Although lithographic techniques have also been reported to guide the VLS growth of ordered SiNW arrays, the complexity of these methods inevitably undermines the advantages of VLS growth method. 

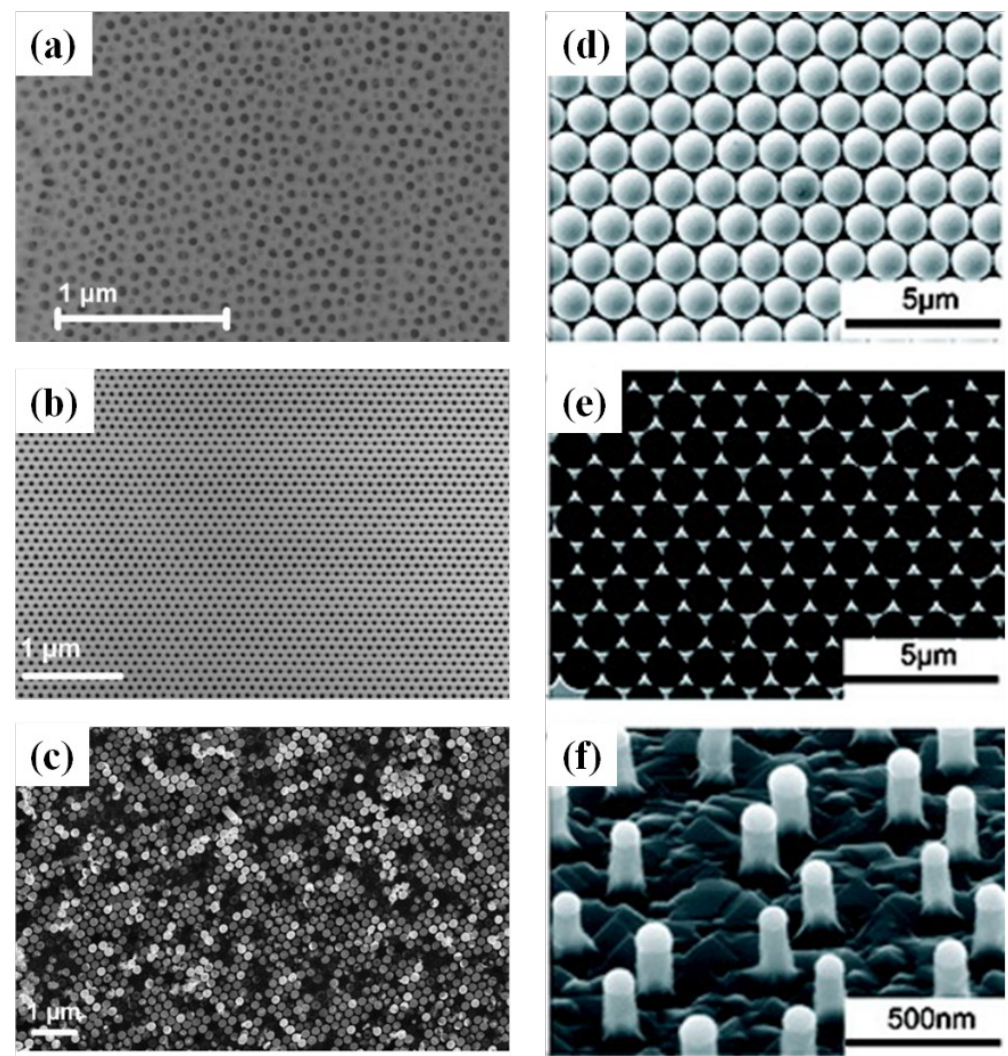

Figure 10 (a) SEM image of porous alumina after simple anodization. (b) SEM image of porous alumina after double anodization followed by widening and opening of the pore's end. (c) Top view of the vertically aligned Si NWs arrays grown with gold catalyst on silicon substrates. (a)-(c) are adapted from [54]. SEM images of arrays of (d) polystyrene nanospheres, (e) Au nanoparticles after removal of nanosphere mask, (f) SiNWs grown with gold templates. a-c reprinted with permission from Ref. [54]. d-f reprinted with permission from [50]. Copyright 2005 American Chemical Society.

Apart from homogenous SiNWs, hybrid nanowires can be grown by the VLS method as well. For example, axial hybrid III-V/Si NWs are grown by MOVPE using gold as catalysts. As shown in Fig. 11, hybrid GaP/Si heterojunction, multijunctions, and superlattice can be obtained by modifying different precursors and growth temperatures [170]. High quality hybrid nanowires with dislocation-free interfaces can be obtained via precise control of the catalyst composition and surface chemistry. The integration of different materials in the SiNWs open new opportunities to achieve high efficiency nanowire solar cells, such as multijunction nanowire solar cells.
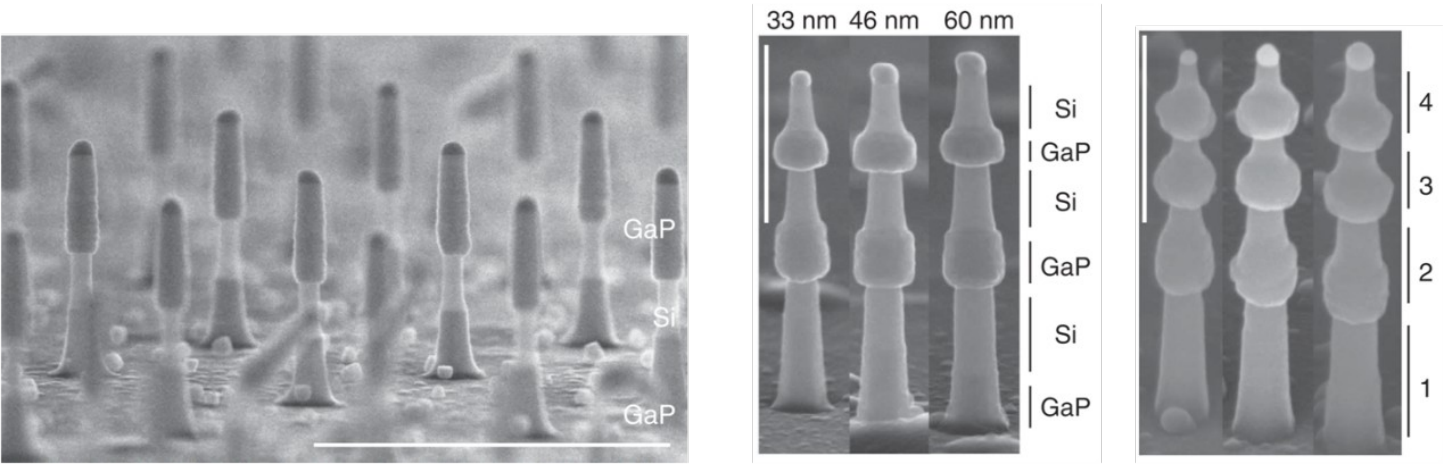
Figure 11 Hybrid GaP/SiNWs: from GaP-Si heterojunction to superlattice. Reprinted by permission from Macmillan Publishers Ltd: [170], copyright 2012.

The intensive studies of VLS growth mode in the last decades lead to precise control in growth of ordered SiNW arrays. However, inevitably, a lot of challenges are presented for VLS growth, and its analogues, of nanowires, including wafer-scale regularity and uniformity, contaminations introduced by catalysts, high thermal budget for precursor decomposition, crystal defects, and complexity in template preparation. In particular, removal of metal catalyst guarantees better performance because Au is a known deep level lifetime killer in semiconductors.

\subsection{Metal-assisted chemical (MAC) etching}

The top-down method is an important complement to self-assembled methods in preparing nanowires in high quality and well-controlled fashion, which normally involves lithographic patterning and etching by chemical or physical processes [171-176]. Although top-down process involves removal of useful materials and, in many cases, time-consuming and expensive processes, it offers desired flexibility and precision in nanowire fabrication. Among various topdown methods, metal-assisted chemical (MAC) etching is particularly attractive and promising for fabrication of SiNW arrays due to its great flexibility, fine control, and low cost. Compared to VLS growth of nanowires, MAC etching does not have obvious limitation on the size, shape, and direction of nanowires. A typical MAC etching procedure involves a noble metal that partly covers a Si substrate and an etchant composed of $\mathrm{HF}$ and an oxidative agent, such as $\mathrm{Fe}\left(\mathrm{NO}_{3}\right)_{3}, \mathrm{KAuCl}_{4}$, $\mathrm{K}_{2} \mathrm{PtCl}_{6}$, and $\mathrm{H}_{2} \mathrm{O}_{2}$. The former serves as a local cathode catalyzing oxidant reduction and injecting holes into the Si substrate. The holes transferred from the "cathode" oxidize the Si substrate. The silicon oxide is then dissolved by the acidic etchant. As the process goes on, the Si beneath the noble metal is etched away while the Si surface without any metal is nearly impacted. As a result, the noble metal "drill" into the Si substrate, forming various Si nanostructures, e.g. SiNWs, depending on the initial surface morphology of the noble metal structure. The MAC etching processes are schematically shown in Fig. 12 (a). The completely solution based process and recently demonstrated electroless MAC etching in vapor ambient at room temperature render greater simplicity and much lower thermal budget than VLS growth method [177-179].

The appearance of MAC etching was much later than the VLS growth mechanism and it can be traced back to the 1990s [180, 181]. In 2000, Li and Bohn reported more systematic studies of MAC etching using different metals for Si substrates of different doping types and levels [182]. Since then, various approaches derived from this method have been widely adopted for SiNWs fabrication and nanoporous structures. For example, Peng et al. pioneered the fabrication of largescale SiNW arrays using MAC etching by immersing Si wafers into the aqueous solution of $\mathrm{AgNO}_{3}$ and $\mathrm{HF}$ [174]. Formation kinetics was investigated in the etching process [183]. As shown in Fig. 12 (a), the metal particles serve as a redox center and function as a short-circuited galvanic cell with a flux of electrons inside the metal particles, while protons would migrate from the anode to the cathode site outside the metal particles [184]. Several other models for the anode reaction have been proposed [185]. The development of MAC etching leads to facile fabrication of SiNW arrays. As shown in Fig. 12(b), large scale arrays with uniform height can be easily obtained by 
varying the concentration of $\mathrm{H}_{2} \mathrm{O}_{2}$ and etching duration [186]. Compared to single step MAC etching, addition of a second etching step lead to further flexibility and functionality of this method. For example, Azeredo et al. fabricated single crystal SiNWs with controlled nanowire surface morphology [37]. First, dewetted Ag nanoparticles are formed on the Si substrate. Over the Ag nanoparticle covered Si surface is deposited a thin layer of Au. A lift-off process is then used to remove the Ag nanoparticles and form Au mesh pattern. After combining with MAC etching, this non-lithographic approach results in SiNW arrays defined by the Au mesh template. The surface profile of the nanowires can be modified by deposition of Au nanoparticles on the nanowire sidewalls and a second MAC etching step. Such a method is efficient to fabricate nanowire antireflection texture of solar cells. Moreover, two-step MAC etching is also useful for removing SiNWs from the Si substrate. An array of high-density SiNWs is strongly bonded to the substrate and is difficult to detach from the wafers. After formation of vertical SiNWs by a first step MAC etching, a second isotropic etching at the bottom of the nanowires destabilizes the nanowire root and helps detach the nanowire arrays [187]. A hot water bath between two same MAC etching steps can also introduce horizontal cracks across the nanowire arrays [188, 189]. The formation of the horizontal cracks via two-step MAC etching facilitates the detachment of the nanowire arrays with uniform length. Another interesting method to detach the nanowires is to add an electrochemical etching step after the MAC etching of SiNWs [190]. The electrochemical etching forms a layer of porous silicon, which can be sequentially removed from the patent substrate by the standard electropolishing method. The detached nanowire arrays can potentially be transferred to an arbitrary substrate, including low cost plastic sheets [191], and glass [192, 193], which is highly desired for low cost solar cells. 

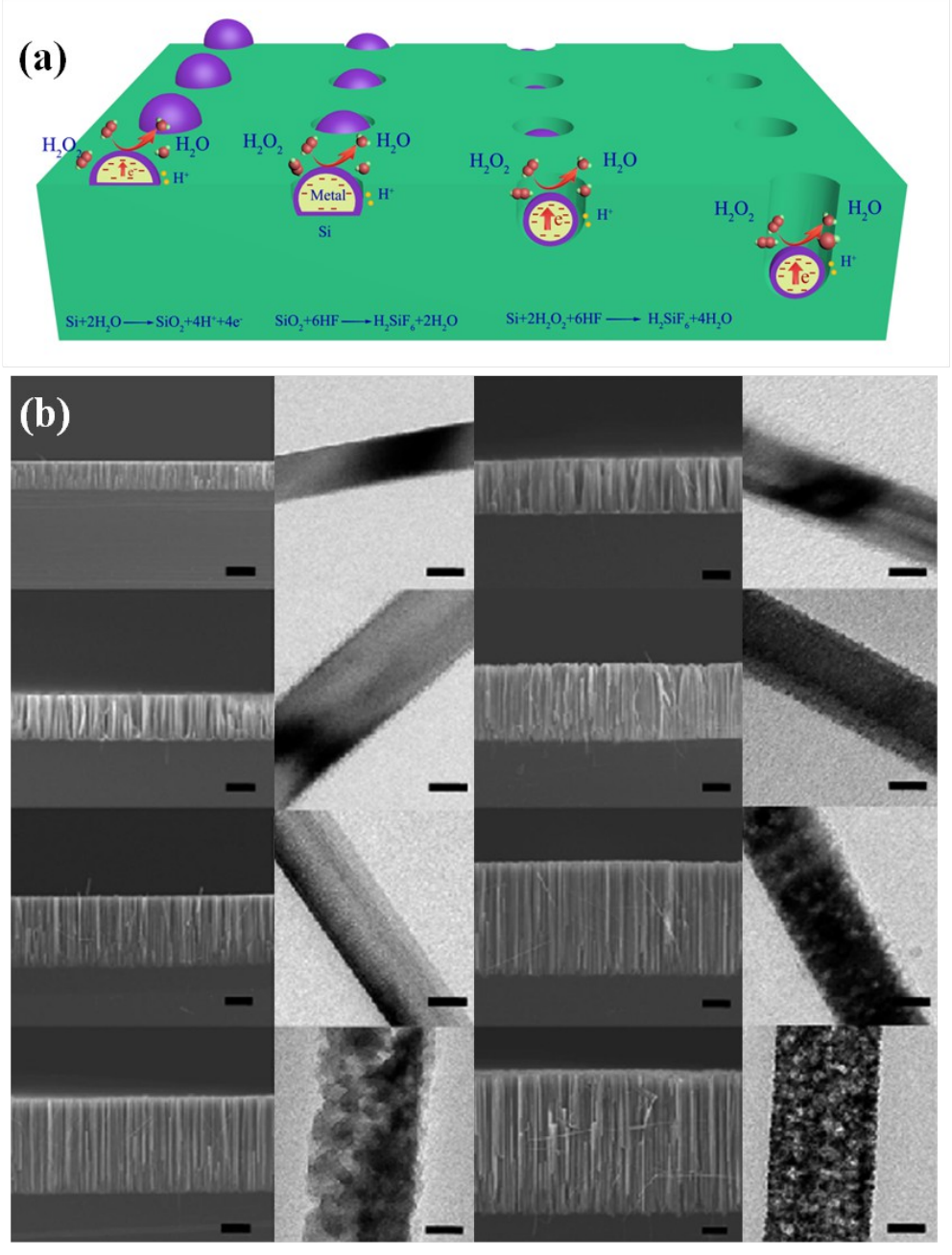

Figure 12 (a) Metal-assisted catalytic reactions on bulk Si surface. (b) SEM and TEM images show the evolution of Si NWs morphology from single crystalline, crystalline/nanoporous, to nanoporous. The engineering of the nanowire morphology is achieved by using different contents of peroxide and etching durations. The scale bars in the SEM and TEM images is $10 \mu \mathrm{m}$ and 60 $\mathrm{nm}$, respectivelyb reprinted with permission from [186]. Copyright 2009 American Chemical Society.

In order to fabricate nanowire solar cells in large scale, the control of the morphology of the nanowire array is essential. The early studies of MAC etching, which is mainly in (100) and (111) substrates, have concluded that the etching is isotropic and nanowires are always vertically formed on the substrate surface $[175,194,195]$. However, Peng et al. found non-vertical etching occurred in (111), (110) and (311) substrates [184]. Chen et al. have reported similar non-vertical etching behavior. The non-vertical etching was later attributed to the back-bond breaking theory. According to the back-bond strength theory, the surface with more back-bonds, which is determined by the crystallographic orientation, is more difficult to remove. The etching anisotropy can be controlled if the back-bond strength can be engineered by choosing the etching conditions. Indeed, the addition of oxidant was found effective to reduce the etching anisotropy (277). As a result, the fabrication of nanowires on non-(100) Si substrates can be manipulated by this method. The intrinsic switch of the nanowire orientations has been observed in literature, which can be 
explained by the variation of oxidant as oxidant being consumed during MAC etching [111, 184, 196]. Meanwhile the etching orientation can also be controlled through the metal catalyst morphology. It has been reported the sufficient large catalytic metal mesh can restrict the movement of the metal catalyst and hence maintain the etching direction [197]. The MAC etching can also be affected by other parameters, including the choice of etchant, type of catalytic metal, etching temperature, illumination, co-solvent, etc [42, 182, 198-206]. The dependence of MAC etching on these parameters undoubtedly renders the great flexibility on precise control of crystal orientation, density, shape and periodicity.

Being similar with SiNWs grown by the VLS growth mechanism, the regularity of nanowire arrays is critical to acquire high efficiency solar cells. The fabrication of ordered nanowire arrays can also be achieved by using templates. Huang et al. assembled a monolayer of polystyrene nanospheres on Si substrate and used the nanospheres as a mask to deposit Ag mesh [175]. Prior metal catalyst deposition, a dry etching (decrease mask size) or deposition of additional materials (increase mask size) step can be introduced to control the size of the nanosphere etching mask. The surface protected by the nanospheres remains unetched and regular nanowires with well controlled density and length and diameters can be achieved. The template assisted MAC etching is schematically shown in Fig. 13. This method has been further optimized by Yeom et al. and other groups to obtain highly ordered SiNW arrays and decouple the diameter and pitch in the arrays [207-209]. However, the nanosphere lithography has a few limitation in preparing waferscale nanowire arrays, including: (1) poor etching selectivity to $\mathrm{Si}$; (2) non-uniform size reduction of the nanosphere mask; (3) inadequate coverage over a large area [210]. Choi et al. employed a "solvent-controlled spin-coating method" to deposit a uniform monolayer of silica nanosphere over large Si wafers. As shown in Fig. 14 (a) and (b), highly uniform silica nanospheres deposited over entire 2 inch wafers. Fig. 14 (c) shows the catalytic metal film with two-dimensional hole arrays with diameters adjusted by reactive ion etching after removal of silica nanospheres. Holes are obtained with high regularity, which further result in well-controlled MAC etching of Si nanopillars, as shown in Fig. 14 (d). Here, a layer of $\mathrm{Ni}$ is also used to improve the adhesion of catalytic metal film and thus produced enhanced lithographical accuracy for fabricating SiNWs arrays in wafer-scale. Recently, a novel two-step template method using nanosphere lithography provides further advancement in terms of controlling the nanowire size, density, and surface arrangement [211]. By employ a different approach, Lin et al. also obtained cone- and needleshaped nanowires with greatly reduced reflectivity [212]. After formation of regular nanowire arrays, additional iterations of selective deposition of Ag nanoparticles and MAC etching can change the nanopillars to tapered nanorods, nanopencils as well as nanocones. The modified nanopillars offers enhanced broadband (400-1000 nm) and omnidirectional (wide angle of incidence between 0 and $\left.60^{\circ}\right)$ absorption even with a ultrathin substrate thickness $(<10 \mu \mathrm{m})$ [213]. 


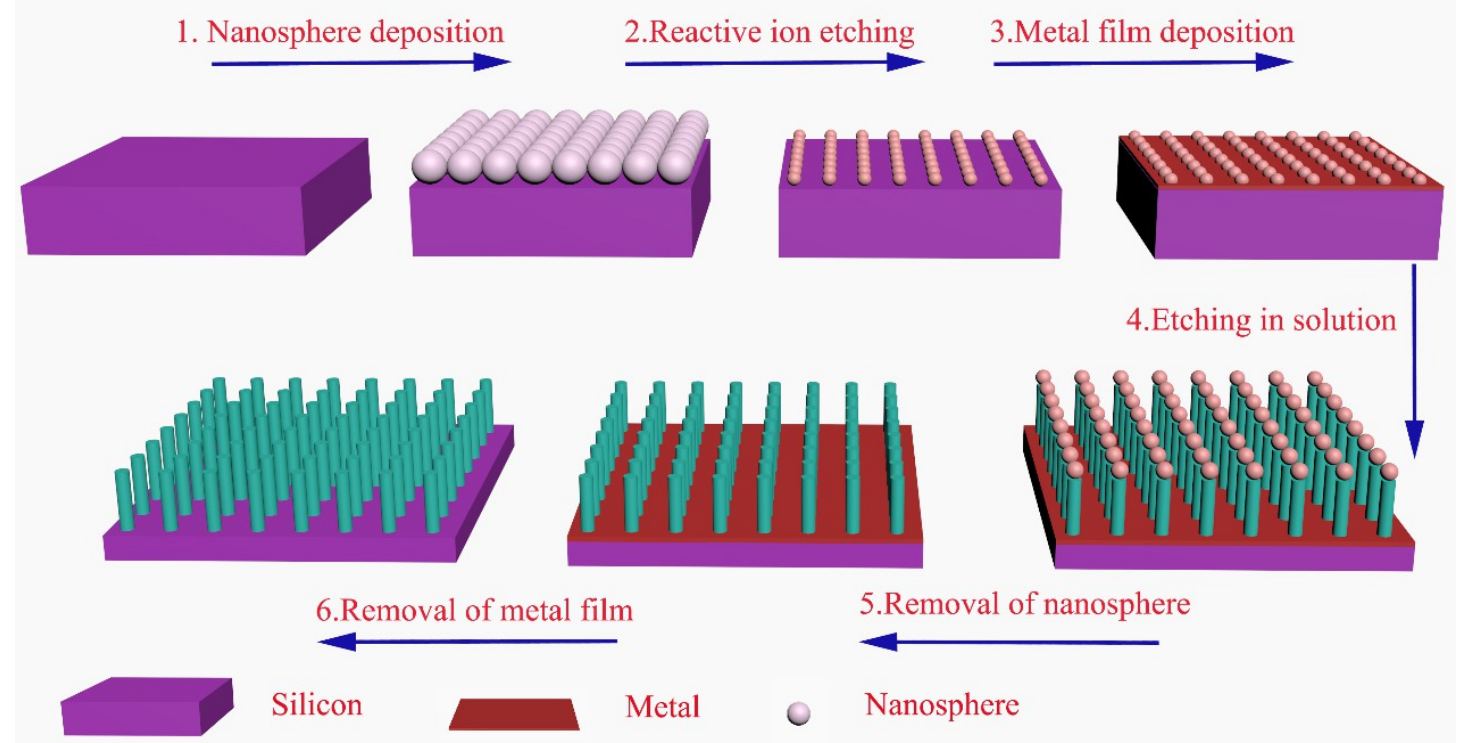

Figure 13 Schematics of fabrication of ordered SiNW arrays using nanosphere lithography with MAC etching.
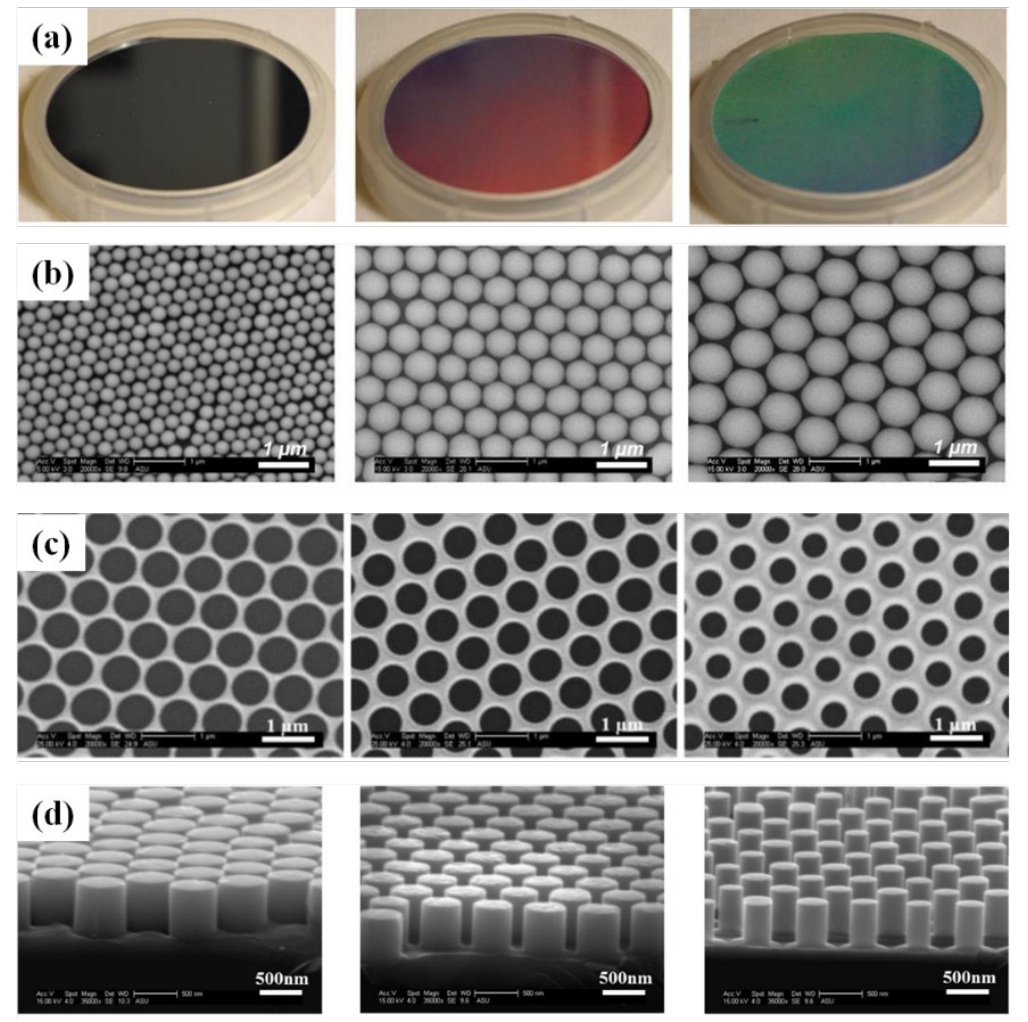

Figure 14 Different sizes ( $310 \mathrm{~nm}, 600 \mathrm{~nm}$, and $840 \mathrm{~nm}$ ) of silica nanospheres spin-coated on 2-in. Si wafers: (a) optical image and (b) magnified SEM images. (c) Ni/Au metal catalyst deposited on Si wafer with different fractional coverage (30\%, 50\%, and 70\%). (d) Silicon nanopillars from panels (c) after MAC etching for $8 \mathrm{~min}, 6.5 \mathrm{~min}$, and $5 \mathrm{~min}$, respectively. Reprinted with permission from [210]. Copyright 2015 American Chemical Society.

Although Si NWs can be fabricated with good regularity and a large range of length and diameter, 
it is quite a challenge to produce small nanowires (diameter $<20 \mathrm{~nm}$ ) as the thickness of the catalytic metal film becomes comparable to the size of the spheres. In addition, even though nanosphere lithography is a self-assembly approach, it involves complicated processing steps, such as sputter deposition, oxygen plasma treatment, and reactive ion etching. Alternatively, Chern et al. used the combination of MAC etching with a superionic-solid-state-stamping (S4) patterning technique to fabricate nanowire arrays with size feature down to $\sim 10 \mathrm{~nm}$ range [214]. Arbitrary stamps can be used to fabricate the nanowire arrays with desired shape and size. However, complex techniques such as e-beam lithography and focused ion beam milling are required to create the stamp, which largely limits its application in large scale. Apart from nanosphere lithography, another extremely useful patterning method is using AAO templates. For instance, Huang et al. used an ultrathin AAO film to create ordered nanohole arrays on a Si substrate [215]. Metal deposition on the nanohole arrays leads to two-dimensional metal film with ordered hole openings. Metal is also deposited in the Si nanoholes but not on the sidewalls. By controlling the metal film thickness, the nanowire diameter can be reduced to sub-10 nm range. Using AAO templates, Kim et al. also proposed the use of $\mathrm{Au} / \mathrm{Ag}$ bilayer mesh to fabricate SiNW arrays with improved quality and etching control compared to Ag-only metal mesh [216].

Instead of forming circular nanowires, Choi et al. used an interference lithography technique to pattern etching masks [176], as shown in Fig. 15 (a). By depositing a layer of catalytic metal and subsequently MAC etching of the substrate, nanowires with cross-section follows the original pattern shape. Fig. 15 (b)-(d) shows arrays of SiNWs, nanofins, and nanowires with oval-shaped cross-section by combining the interference lithography technique and MAC etching. Jeon et al. also reported a facile and cost-effective method to fabricate SiNWs with unconventional shapes using holographic lithography to create the metal catalyst patterns [217]. Combination of blockcopolymer lithography and MAC etching is also another important method to fabricate ordered nanowire arrays in large scale at low cost [218]. 
(a)
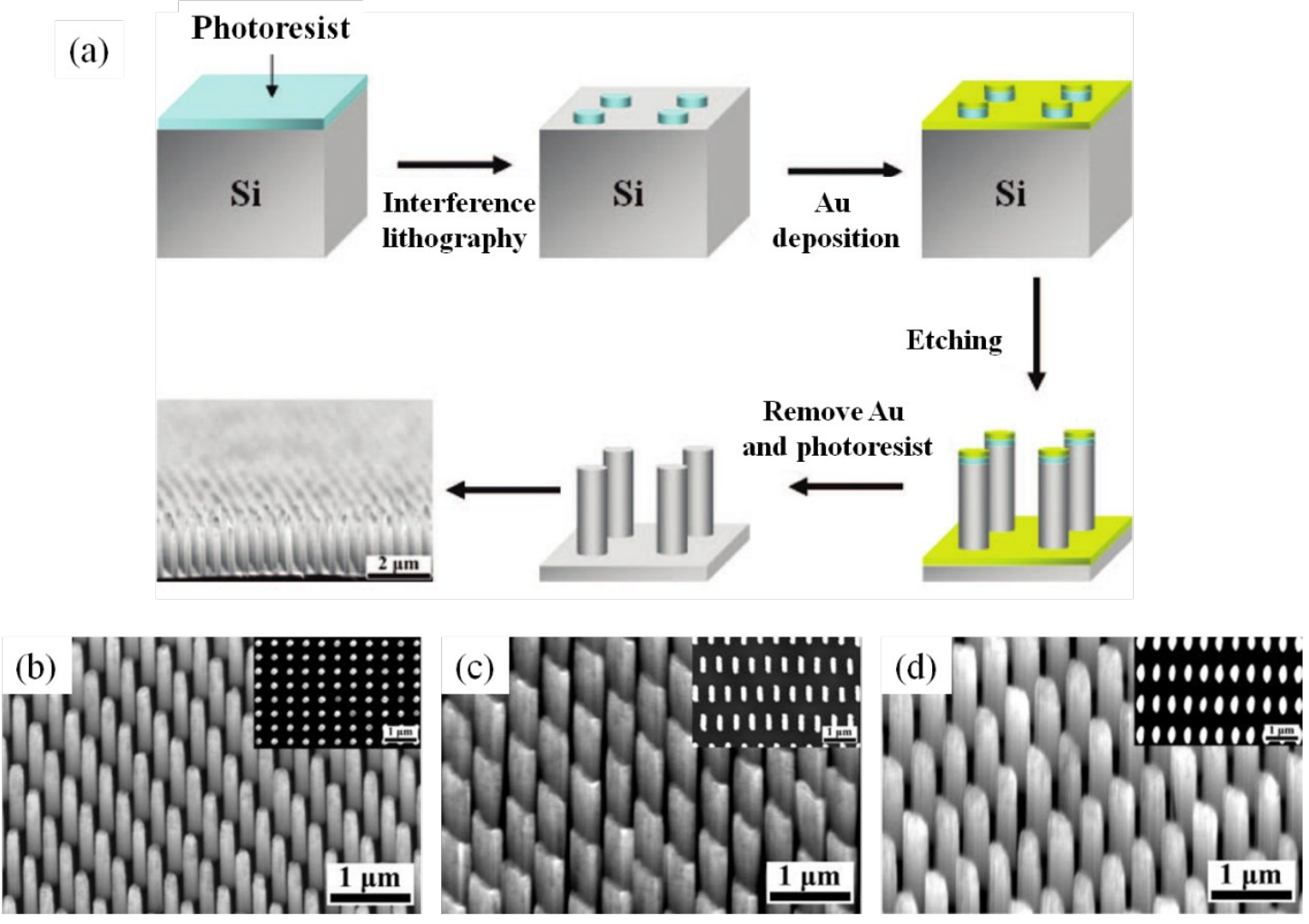

Figure 15 (a) Schematic diagrams of MAC etching of SiNW arrays with interference lithography patterned template. SEM images of (a) SiNWs, (b) Si nanofins, and (c) SiNWs with oval cross sections, obtained after MAC etching of the interference lithography patterned template. Reprinted with permission from [176]. Copyright 2008 American Chemical Society.

MAC etching method consumes nearly the same amount of materials with the planar counterparts, which seems to breach the requirement of cost-effective solar cells. However, the for ultra-thin absorbing layers the light-trapping effects dominate, while for thicker cells that already absorb a large fraction of the incident light, the recombination effect is more important [78, 219]. Therefore, when the thickness of Si substrate decrease to several microns, additional nanowire atop (several microns long) enhance the path length of incident solar radiation. Moreover, as a complementary method for preparing nanowires, MAC etching do not involve in expensive vacuum, plasma generation, ion optics etc. necessities in VLS or dry etching process. The intensive investigations in MAC etching of SiNWs in the last ten years have already led to significant advancement in controlling the nanowire quality, orientation, aspect ratio, diameter, length, etc. In combination with various template patterning methods, highly regular array of nanowires in large scale can be readily obtained. However, there are still some opening questions remain: The influence of the doping type and doping level of Si substrate, surface state of Si substrate, and the type and morphologies of the noble metal catalyst during the hole injection process remains incompletely understood.

\subsection{Fabrication of SiNW arrays via dry etching of patterned silicon}

Direct dry etching is another important top-down method to fabricate SiNWs with high quality and uniformity. Common dry etching techniques include reactive ion etching (RIE), inductively 
coupled plasma (ICP)-RIE, chemical assisted ion beam etching (CAIBE). The dry etching process has been well-established and compatible with standard silicon microfabrication techniques. Compared with VLS growth mechanism and MAC etching, the dry etching of silicon is more straightforward. In order to fabricate nanowire arrays, dry etching is generally paired with predefined templates. The standard optical lithography is a mature technique with high throughput and low cost but not suitable for nanoscale fabrication. In-situ formation of etching mask has been reported to form SiNWs. An un-masked one step etching of Si with reactive gases comprising of argon, hydrogen, methane, and silane generates uniform and high- density nanotip arrays over a large area [95]. The in-situ formation of SiC nanoclusters via the reaction between silane and methane is attributed to the self-masked dry etching. Huang et al. adopted this method to fabricate Si nanotips over an entire 6-inch wafer, as shown in Fig. 16 (a) [92]. Fig. 16 (b) and (c) shows that $\mathrm{Si}$ nanotips with high aspect ratio are obtained by using a high-density electron cyclotron resonance plasma etching. Fig. 16 (d) compares the absorption spectra of the Si nanotip sample shown in Fig. 16 (c) and a crystalline silicon from ultra-violet to near-infrared region. As introduced in the previous section, the reflection of the nanotip/wire structures can be reduced below $1 \%$ over a wide spectral range from visible to mid-infrared and in a wide range of incident angles from $5^{\circ}$ to $60^{\circ}$ [92]. This method can be used to fabricate randomly distributed nanowire arrays in large scale with relatively low cost.

\section{(a)}

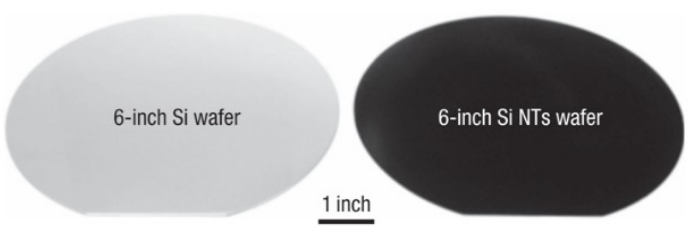

(b)

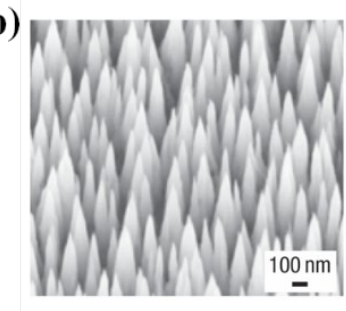

(c)

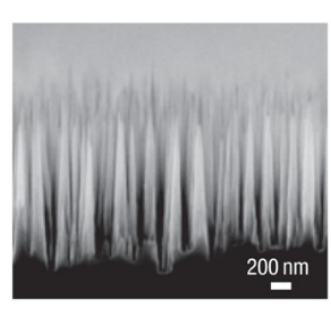

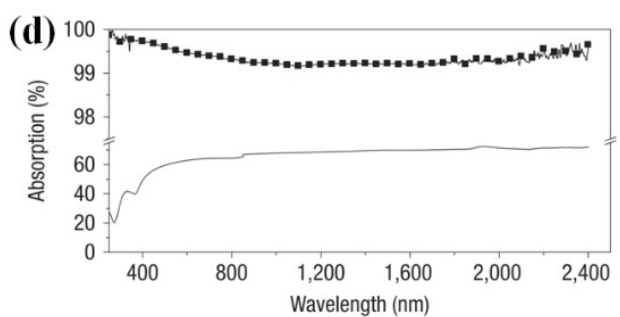

Figure 16 (a) Photographic images of 6-inch polished silicon wafers without (left) and with Si nanotips (right). (b) and (c) Tilted top view and cross-sectional view of SEM images of Si nanotips. (d) Absorption spectra of the Si nanotips (b, c) (filled squares) and crystalline silicon (solid line) from ultraviolet to near infrared region. Reprinted by permission from Macmillan Publishers Ltd: [92], copyright 2007.

However, this method has poor control of structural parameters, such as density and size. As discussed earlier, in practical applications, ordered nanowire arrays with well controlled structural parameters are favored. Ordered etching mask can be fabricated by lithographic techniques, such as e-beam lithography [220], which is expensive and time-consuming and thus not suitable for fabrication of nanowires in large arrays. Nanoimprint lithography in combination with reactive ion etching has been used to fabricate SiNW arrays [173]. This method can allow fabrication of welldefined nanowire arrays with precise control in position, density, and size. Optimum etching parameters can lead to uniform vertical nanowires with high aspect ratios. Broadband and 
omnidirectional antireflection of Si nanocylinders has also been achieved by using a soft-imprint fabrication technique with reactive ion etching [221]. Although soft-imprint can be compatible to large-scale and high-fidelity patterning but the fabrication of stamps normally involves complicated and expensive top down processes. The one major drawback of the imprint methods is the high cost of the stamp template, particularly for a large size imprint area. Other disadvantages of nanoimprint lithography include the formation of a large number of defects and short lifetime of the stamp template [222]. A self-powered parallel electron lithography technique was developed to form SiNW arrays with feature size down to sub-35-nm [223, 224]. A large-area planar electron emitting thin film is used to expose e-beam resist through a stencil mask. Wellordered Si nanopillar arrays have been fabricated with superior absorptance ( 99\%) over the entire visible range. However, the use of the radiative thin film emitter and stencil mask complicated the process and incurred additional cost. As a result, self-assembled templates with low cost, including nanosphere lithography, block copolymer lithography, are normally used to fabricate ordered nanowire arrays along with dry etching.

Kuo et al. employed nanosphere lithography to create metal masks and a double layer nanosphere lithography leads to the formation of ordered metal nanoparticle arrays [225]. However, the surface coverage is not close packed and large-scale uniformity is hard to obtain due to formation of different domains. The uniformity and coverage of nanosphere deposition can be improved by using the Langmuir-Blodgett method, which is capable of preparing a monolayer of nanoparticles over wafer-scale. Hsu et al. reported uniformly dispersed close packed $\mathrm{SiO}_{2}$ nanospheres over an entire 4-inch wafer by Langmuir-Blodgett method [226]. Figure 17 shows the schematic diagram of the nanowire fabrication processes as well as SEM images obtained. The $\mathrm{SiO}_{2}$ nanospheres used in the Langmuir-Blodgett deposition is functionalized with aminopropyl methyldiethoxysilane to prevent aggregation. The well-dispersed $\mathrm{SiO}_{2}$ nanospheres are shown in Fig. 17 (a). Similar to nanosphere lithography patterned MAC etching, the diameter and periodicity of the nanowire arrays can be tuned with an additional etching step of the $\mathrm{SiO}_{2}$ nanospheres (Fig. 17 (b)). Si nanopillars are then formed by selective and anisotropic dry etching of the Si substrate masked by the $\mathrm{SiO}_{2}$ nanosphere array, as shown in Fig. 17 (c). After formation of Si nanopillars, the nanosphere mask can be easily removed by HF etching (Fig. 17 (d)). In addition to obtaining uniform nanosphere templates in large scale, Hsu et al. demonstrated formation of tapered nanowires by modifying the dry etching process. After formation of Si nanopillars, isotropic etching is performed on the nanopillars, which undercuts and sharpens the nanopillars. Lately, Rahman et al. confirmed the promise of these tapered SiNW structures in solar cells [227]. Using the tapered SiNWs as the surface textures of a crystalline silicon solar cell, the short circuit current is improved from 22 to $33 \mathrm{~mA} / \mathrm{cm}^{2}$ due to the broadband antireflection much higher than that of the solar cell coated with optimized silicon nitride antireflection layer (28 $\mathrm{mA} / \mathrm{cm}^{2}$ ). The photocurrent of the solar cells with tapered nanowires is nearly constant even at incident angles up to $60^{\circ}$.

While dry etching is a well-developed top-down technique in semiconductor industry for patterning, it tends to create surface defects of the etched sidewalls [228]. These surface defects are detrimental in nanowire devices because of the large surface to volume ratio. Therefore, postetching processes, such as passivation, should be applied to minimize the surface recombination of 
nanowire solar cells.

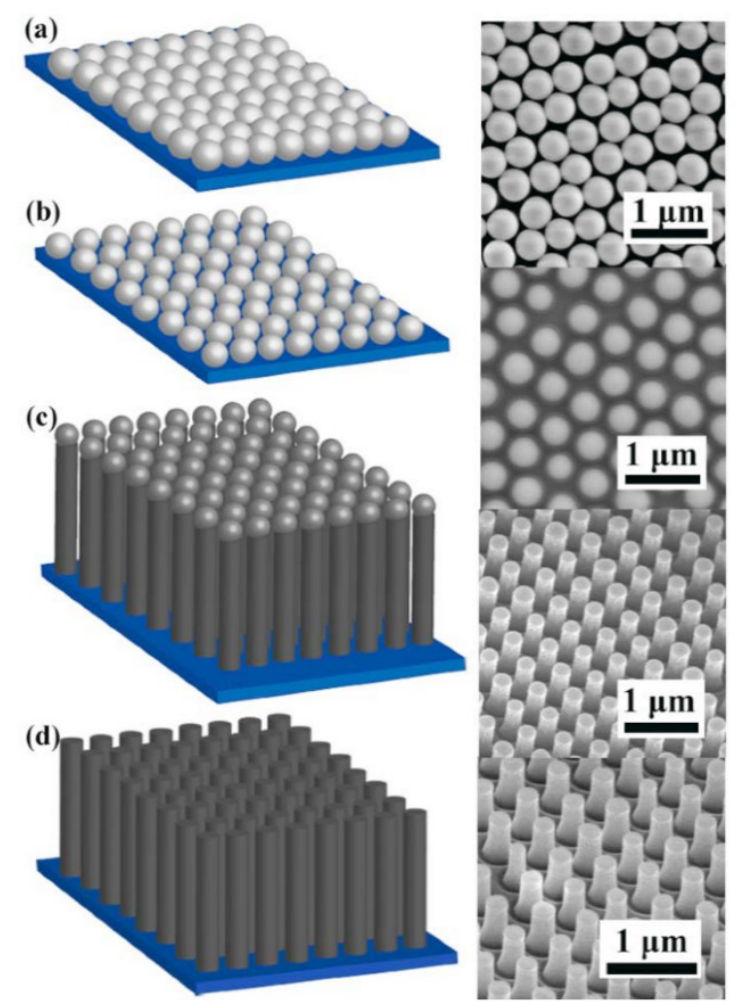

Figure 17 Fabrication sequences of Si nanopillars using nanosphere lithography and dry etching. (a) Deposition of the silica nanospheres by Langmuir-Blodgett method. (b) Size reduction of the nanospheres by isotropic dry etching. (c) Anisotropic etching of Si into pillars masked by nanospheres by reactive ion etching. (d) Removal of the silica nanosphere mask by HF etching. Reprinted from Ref. [226] with the permission of AIP Publishing.

To summarize this section, we draw a table for comparing different characteristics of VLS, MAC etching and dry etching, including size control, aspect ratio, orientation, morphology, cost, etc.

Table 2 Different characteristics of SiNWs by VLS, MAC etching and dry etching method

\begin{tabular}{|c|c|c|c|c|c|c|c|c|}
\hline Method & Diameter & $\begin{array}{l}\text { Aspect } \\
\text { ratio }\end{array}$ & Orientation & Morphology & Cost & $\begin{array}{l}\text { Longitudinal/ } \\
\text { radial } \\
\text { heterojunction }\end{array}$ & damage & $\begin{array}{l}\text { Siedwall } \\
\text { smoothness }\end{array}$ \\
\hline VLS & $\begin{array}{l}\text { Determined } \\
\text { by size of } \\
\text { catalysts }\end{array}$ & High & $\begin{array}{l}\text { Depends } \\
\text { on the } \\
\text { diameter of } \\
\text { nanowire }\end{array}$ & $\begin{array}{l}\text { Wires with } \\
\text { circular } \\
\text { cross- } \\
\text { section }\end{array}$ & High & Easy/Impractical & $\begin{array}{l}\text { Metal } \\
\text { contamination } \\
\text { or twin fault }\end{array}$ & Smooth \\
\hline $\begin{array}{l}\text { Mac } \\
\text { etching }\end{array}$ & $5 \mathrm{~nm}-1 \mu \mathrm{m}$ & High & $\begin{array}{l}\text { Determined } \\
\text { by crystal }\end{array}$ & $\begin{array}{l}\text { Determined } \\
\text { by etching }\end{array}$ & Low & Hard/Impracticable & None & $\begin{array}{l}\text { Smooth or } \\
\text { rough }\end{array}$ \\
\hline
\end{tabular}




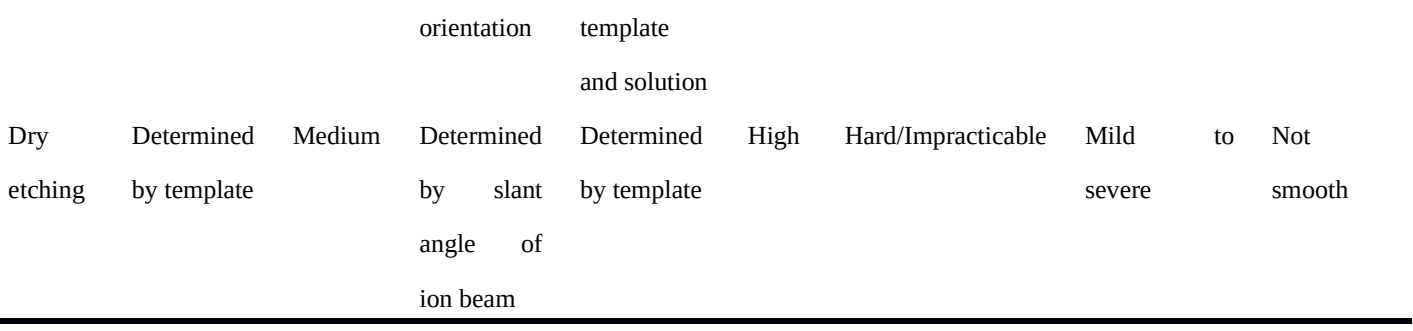

\section{SiNW-based solar cells}

\subsection{Main electrical consideration for NWs-based PV devices}

\subsubsection{Theoretical modelling for efficient radial $p-n$ junction solar cell}

After obtaining favorable optical properties, the understanding of electrical properties is essential to achieve the final solar cells. For many years the efficiency of low costs solar cells has been limited to the detailed balance limit proposed by Shockley and Queisser, which takes the intrinsic thermodynamic losses into account in the cells. To have a better understanding of the mechanism behind the SiNWs, here we review electrical physics of the radial SiNW-based solar cells which differs from the axial one, favored because the enhanced light trapping and short carrier collection lengths to facilitate collection rooted in radial core-shell geometry. While excited carriers of the planar one has comparable distance to be extracted, the travel distance of radial device is as short as the radius of the wire. In this structure, the photogenerated electron-hole pairs are separated by the built-in electric field, and then drift apart. While light is absorbed in axial direction, the carriers are extracted in the radial direction.

Two factors must be taken into account when achieving large enhancement efficiency. First, the diffusion length of minority carriers must be long to guarantee extraction of most light-generated carriers before recombination. Next, the rate of carrier recombination in the depletion region must be lowered. For silicon, carrier life time above 10 ns is enough. Harry A. Atwater modelled the planar and radial p-n junction nanorod solar cells with consideration of Shockley-Read-Hall recombination and surface recombination [78], as shown in Fig. 18 (a) and (b). For $100 \mu \mathrm{m}$ thick silicon solar cell, the short-circuit current density $\boldsymbol{J}_{\text {sc }}$ in the radial p-n junction nanorod geometry is $\approx 38 \mathrm{~mA} / \mathrm{cm}^{2}$ for thin (100-nm radius) rod, decreasing slightly to $\approx 35 \mathrm{~mA} / \mathrm{cm}^{2}$ as the rod radius increases. However, the $\boldsymbol{J}_{\boldsymbol{s c}}$ decreased from 34 to $4 \mathrm{~mA} / \mathrm{cm}^{2}$ as density of recombination centers increased in a planar cell. $\boldsymbol{V}_{\boldsymbol{o c}}$ is independent of cell thickness in planar cell, and for the radial p-n junction case, the $\boldsymbol{V}_{\boldsymbol{~}}$ decreases as the cell thickness increases because of the increasing junction area in radial architecture. In Fig. 18 (c), (d)), a lower efficiency rooted in a high quasineutralregion trap density in spite of the trap density in the depletion region. On the other hand, for the radial p-n junction, the efficiency stays high if the trap density in the depletion region is low. The SiNW cell maintains higher efficiency than planar cell when the $L_{n}$ and thickness are identical. For instance, the maximum efficiency of the radial nanorod geometry with $L_{n}=100 \mathrm{~nm}$ is $11 \%$ while its counterpart is only $1.5 \%$, indicating that the the SiNWs are capable of cutting down the costs of 
materials dramatically via using mg-Si. Data from experiments support this. Heayoung P. Yoon et al. report enhanced conversion efficiencies for pillar array solar cells fabricated from crystalline silicon with short minority carrier diffusion lengths [229]. NW solar cells and their planar counterpart were fabricated on $\mathrm{p}^{+}$crystalline Si substrate with short minority carrier diffusion length of $L_{n} \sim 10 \mu \mathrm{m}$ and $1 \mu \mathrm{m}$. Harry A. Atwater's group demonstrates enhanced absorption and carrier collection in radial SiNWs for photovoltaic applications [230]. They further demonstrate that the light absorbed by SiNWs can be collected with a peak external quantum efficiency (EQE) of 0.89, showing broadband, near-unity internal quantum efficiency (IQE) for carrier collection through a radial semiconductor/liquid junction at the surface of each wire.

The proof-of-concept radial junction solar cells with a low diffusion length to $10 \mu \mathrm{m}$ still obtain PCE of $8.7 \%$ compared with $4.6 \%$ in planar counterparts [78, 130]. Additionally, FDTD simulation shows that radial wires show distinct advantages over axial wires, exhibiting high internal quantum efficiency (IQE) values nearly independent of surface recombination [231]. But the challenge is the control of doping depth and uniformity of shell layer. Those tiny wires can easily become completely doped rather than only the outer region. Additionally, a uniform coating of a very thin n-shell on high aspect ratio NWs is difficult to realize. By using charge-transfer and confinement mechanism, modulation doping can be achieved in radial junction nanowires [232]. Chemical vapor deposition is also applied on radial junction nanowire synthesis [233, 234]. It has potential to produce significant improvements in efficiencies in low diffusion length and low depletion region recombination materials. 
(a)

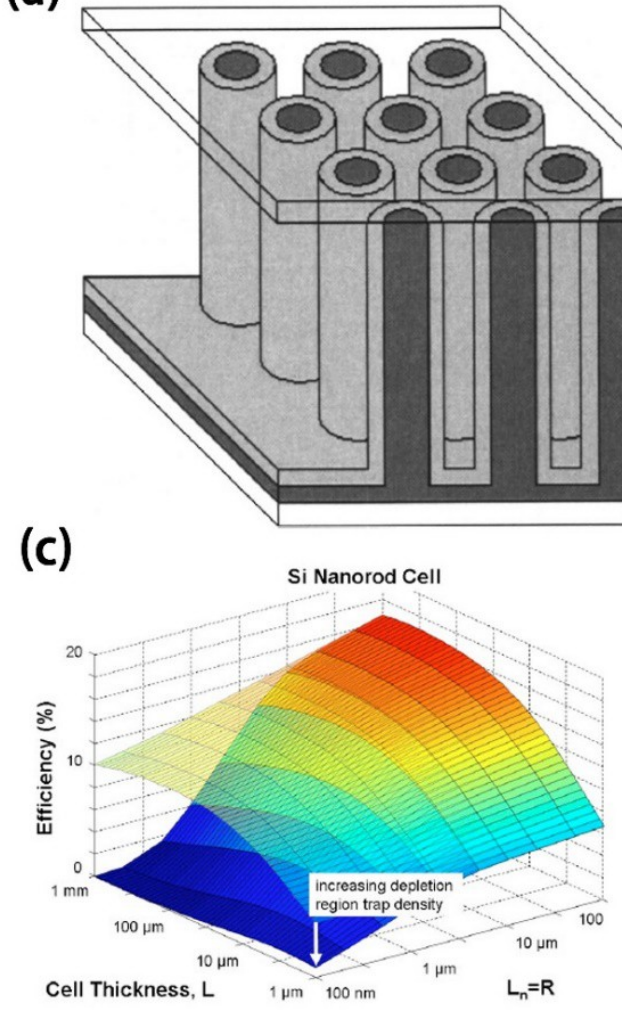

(b)
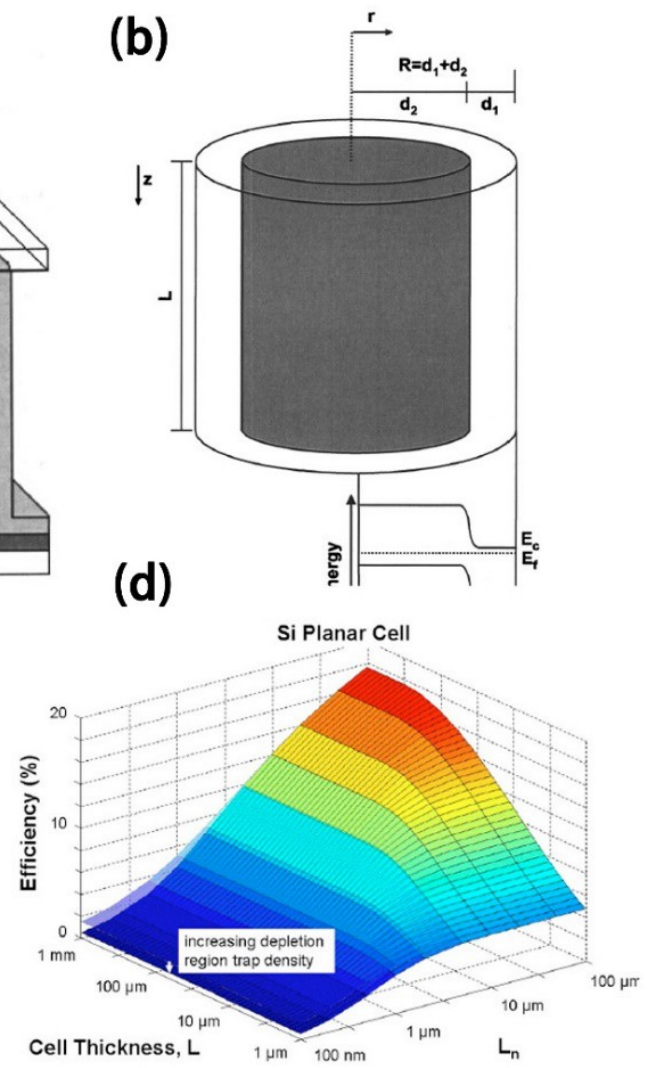

Figure 18 (a) Schematic of cross-section of the radial p-n junction nanowires. (b) The energy band diagram of single nanowire. (c) Efficiency VS cell thickness $L$ and minority electron diffusion length $L_{n}$ for SiNW cells and $R$ (radius of NWs) is $L_{n}$. (d) Efficiency VS cell thickness $L$ and minority electron diffusion length $L_{n}$ for Si planar cell. The simulated light is incident on the top and the grey area is $\mathrm{n}$ type, and the dark grey is $\mathrm{p}$ type. Reprinted from Ref. [78] with the permission of AIP Publishing.

\subsubsection{Surface recombination}

Despite their excellent light trapping capacity, surface recombination (SR) is a major concern due to high surface-to-volume ratio in SiNW. Exposure to ambient air induces unwanted and uncontrolled interfacial states in the Si band gap [235]. Therefore, it is essential to explore models and methods to predict and control the surface physical characteristics of oxide-free SiNW when considering surface recombination [236].

Fortunately, the surface defects can be conquered by via and inorganic organic surface bonds , such as $\mathrm{Al}_{2} \mathrm{O}_{3}$ [56, 237, 238], a-Si [239, 240], $\mathrm{SiN}_{\mathrm{x}}$ [241-243], thermal $\mathrm{SiO}_{2}$ [235, 241, 244], insitu chlorine [239, 245-247], and UV ozone [248], etc. By an approach of dielectric passivation, high performance SiNW based solar cells with a conversion efficiency of $17.11 \%$ and a large size of $125 \times 125 \mathrm{~mm}^{2}$ was reported [249]. The recombination rate at the surface of the device is valued by surface recombination velocity (SRV). The predicted PCE drops gradually from $17.3 \%$ to $15.2 \%$ with increasing SRV varying from 0 to $10^{3} \mathrm{~cm} / \mathrm{s}$ while the further increase of SRV to $10^{5}$ $\mathrm{cm} / \mathrm{s}$ makes PCE plummet to 6.1\% [250]. Yu et al. performed microscopic three-dimensional 
simulations to study axially and radially arranged III-V-based structures to understand the impact of surface recombination on the photovoltaic performance in analogy with SiNWs structure [247]. Under a high SRV rate $\left(10^{6} \mathrm{~cm} / \mathrm{s}\right)$, the efficiency of axial p-n-junction nanowires drops by $63.2 \%$, while the efficiency of radial p-n-junction nanowires falls only by 24.1\%. Fig. 19 (a) depicts the recombination rates at the wire surface along the direction parallel to the nanowire central for both axial and radial structures at the SRV level of $10^{6} \mathrm{~cm} / \mathrm{s}$. It can be seen that, for the radial structure, the overall surface recombination rate is lower than that of the axial p-n structure, excluding a small region. Since minority carrier density plays a critical role in surface recombination rate, as plotted in Fig. 19 (b). While the minority carrier concentration is as high as approximate $10^{22} \mathrm{~cm}^{-3}$ around the center of the radial structure nanowire, the minority carrier concentration at surface is significantly smaller, thus benefiting from the lower SR rate. The holes in n-doped region diffuse toward the depletion region fleeing from the surface due to the proximity of the nanowire surface to the p-n junction in a radial structure. On the other hand, the nanowires on axial structure surface connected with the bulk incur charge accumulation and further lead to strong surface recombination.

The SiNWs depends strongly on passivating functional groups and the passivated areas, and it would generally modify the electronic properties of SiNWs. Man-Fai et al. simulated modulated work function of silicon nanowires with different chemical passivations by Density Functional Theory (DFT) method [251]. Here, the surface effects for fluorine passivated SiNW (F-SiNW), hydroxyl passivated SiNW (OH-SiNW) and hydrogen passivated SiNW (H-SiNW) with different percentage of coverage are investigated. The energy of work function (WF), vacuum potential (VP) and Femi level (FL) as functions of surface composition is shown in Fig. 19 (c). The highest work function among three materials is the F-SiNW, which can be attributed to the high electronegativity of $\mathrm{F}$ atom. Electrons from the surface of the F-SiNWs are withdrawn by high electronegativity $\mathrm{F}$ atoms, and thus introduce the increased charge density around the $\mathrm{F}$ atoms; the negative end of the induced dipole thus enhanced the surface dipole, pointing towards the vacuum. Therefore, thus leads to the work function increase. Fig. 19 (d) provides a better understanding of the phenomenon that $\mathrm{OH}-\mathrm{SiNW}$ s with coverage of $50 \%$ and $100 \%$ have a smaller work function than that of the $\mathrm{H}-\mathrm{SiNW}$ despite an $\mathrm{O}$ atom is more electronegative than a $\mathrm{H}$ atom. It presents the surface structure of SiNWs with 100 coverage of H, F, and, OH respectively, accompanying with zigzag Si-Si bonds. In H-SiNW case, the inner core Si-Si bonds is $2.393 \AA$, slightly longer than the surface Si-Si bonds (2.385-2.378 $\AA$ ); the F-SiNW is contracted or elongated, as shown in the figure. However, it is quite different for the $\mathrm{OH}-\mathrm{SiNW}$. The $\mathrm{OH}$ introduces the conjugate-liked zigzag Si-Si bonds, indicating that there is nothing modified by $\mathrm{OH}$ passivation on the surface. This can be explained by the electron resonance effect of $\mathrm{OH}$ group directed to the surface of SiNW.

Overall, according to their simulation, there we can conclude that three prerequisites must be considered for achieving desirable passivation: first, highly electronegative materials is preferred and materials that can result in a strengthened surface dipole and conjugated-like surface and core bonds within SiNW must be avoided; second, the stability after passivation; last, charge density on the surface after passivation because the increased charge density on the surface causes a reduction of work function. Fig. 19 (e) demonstrates the calculated work function of commonly 
used semiconductors with clear surface at different p doping levels, compared with Man-Fai's work [251]. The high doping level determines the high work function which expels electrons from surface to inner and thus prevents surface recombination. However, the highly doped semiconductor may lower carrier mobility so that too low to respond, not suitable for high-speed device. Therefore, there are tradeoffs between doping and lowering of the surface recombination.

Meanwhile, experiments from Xin Wang et al. show that surface passivation and performance degradation connected with minority carrier lifetime [252]. As shown in Fig. 19 (f), C@SiNW array samples presents improved lifetime as high as $21 \mu$ secause the surface recombination is mitigated by a conformal carbon layer because the carbon coating is able to withstand hostile environments, such as aggressive chemicals and irradiation. Termination of SiNWs with alkyl molecules through covalent $\mathrm{Si}-\mathrm{C}$ bonds can provide long term stability against oxidation of the $\mathrm{Si}$ NW surfaces [236]. These verify that conformal carbon coating is an alternative promising passivation route for SiNW solar cells. D. Yu's group reported a long minority electron diffusion (dominant contribution for photocurrent in this case) length up to $2.7 \mu \mathrm{m}$ in bridged p-type SiNW with a diameter of $100 \mathrm{~nm}$ by using scanning photocurrent microscopy (SPCM), which corresponds to a low surface recombination velocity of $70 \mathrm{~cm} / \mathrm{s}$ [253]. Though the exotic light trapping for long length NWs, it degrades the performance because the increased surface recombination. Mrinal Dutta and Naoki Fukata, showed that the solar cells with $7.8 \mu \mathrm{m}$-long NW arrays presenting the most degraded performance although they have the lowest reflection because the advantage of very lower reflection at shorter wavelength is unable to compensate the disadvantage of increased rough surface that supports surface recombination [248]. The increased defects are mainly from increased dangling bonds in long NWs. They applied low-temperature UV ozone treatments to passivate the defects. Results show that the intensity of the electron spin resonance (ESR) defect signal decreases with increasing UV ozone treatment temperature because the atomic oxygen diffuses into the polycrystalline p-type layer and passivates the dangling bonds during the UV ozone treatment.

Apart from application of various dielectric passivation coatings, other methods such as multiscale surface texture, optimizing nanostructure density, novel silicon nanostructures with low surface area enhancement, control over the nanostructure height, and reducing emitter doping concentration may work [254]. The double-edged high surface-to-volume ratio in nanowire solar cells introduce exotic properties for potential applications. On the other hand, it impairs the photogenerated current. Therefore, the design principle for curbing surface recombination is to passivate the defects. Benefiting from the mature and advanced integrated circuit industries, the surface recombination can be limited at the greatest extent by abovementioned methods. 
(a)

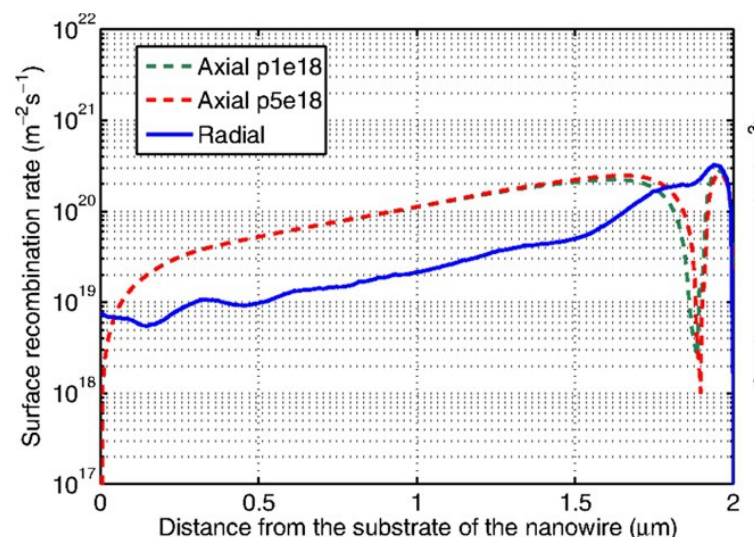

(c)

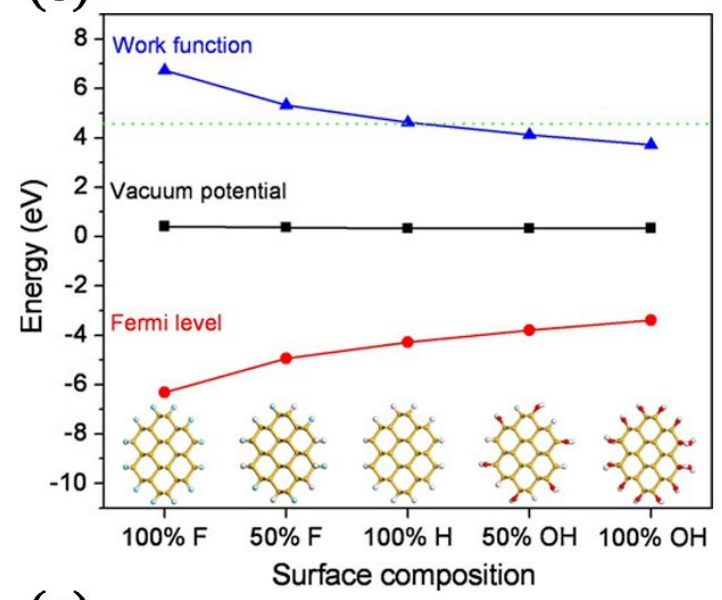

(e)

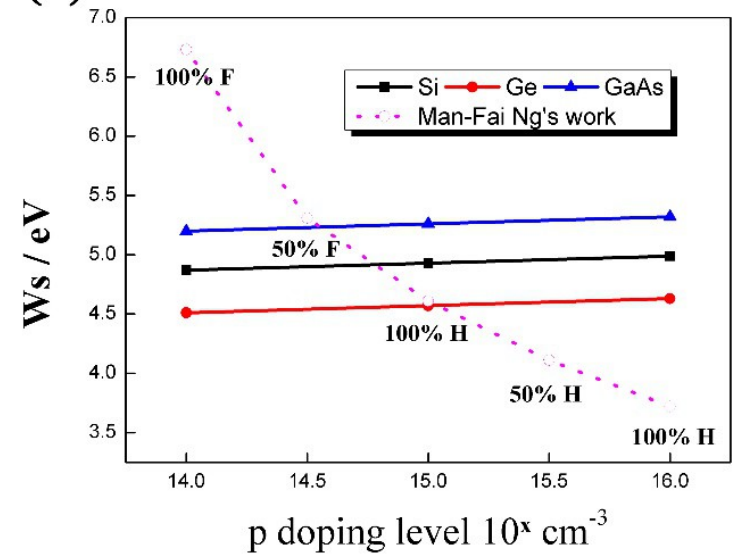

(b)

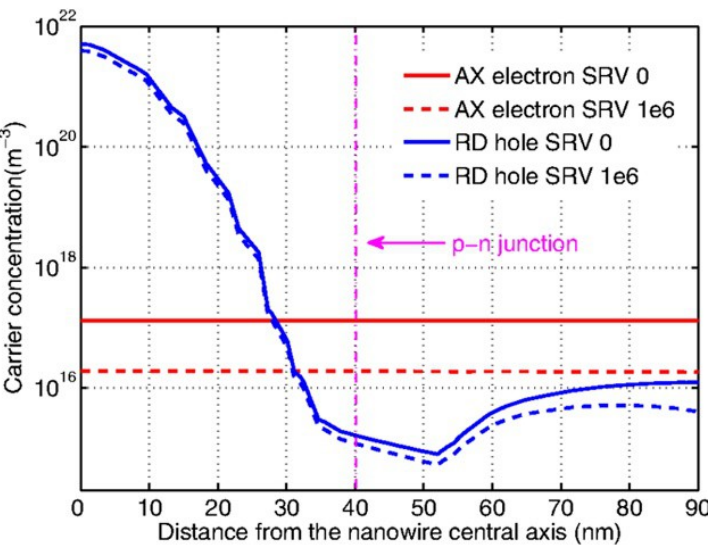

(d)

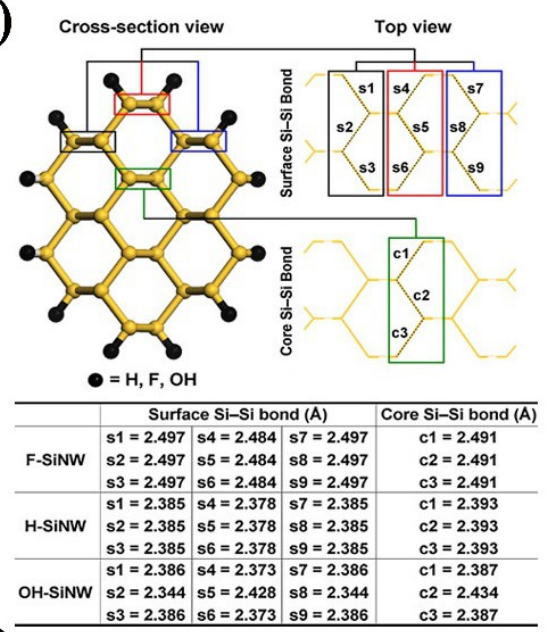

(f)

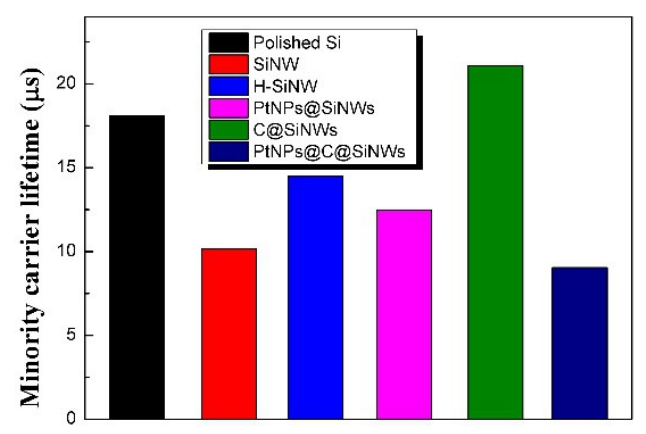

Figure 19 (a) Surface recombination rate comparison of axial p-n-junction nanowire $\left(5 \cdot 10^{18} \mathrm{~cm}^{-3}\right.$ $\mathrm{p}$-doping) and radial $\mathrm{p}$-n-junction nanowire n-region $\left(5 \cdot 10^{18} \mathrm{~cm}^{-3} \mathrm{n}\right.$-doping), at $0 \mathrm{~V}$ bias. For the radial junction case, the SR is localized in the n-doped shell, while for the axial junction case the major contribution comes from the p-doped region. Hence, only these regions were considered for further analysis. (b) Surface minority carrier concentration comparison of the axial (AX) p-njunction nanowire $\left(5 \cdot 10^{18} \mathrm{~cm}^{-3} \mathrm{p}\right.$-doping) p-region and the radial $(\mathrm{RD}) \mathrm{p}$-n-junction nanowire $\mathrm{n}$ region ( $5 \cdot 10^{18} \mathrm{~cm}^{-3} \mathrm{n}$-doping), cut made at $1.3 \mu \mathrm{m}$ height, at $0 \mathrm{~V}$ bias. (c) A plot of work function, vacuum potential and the Fermi level as functions of surface composition for the H-SiNW, FSiNWs and OH-SiNWs. The green dotted line indicates the work function of the H-SiNW for 
comparisons. (d) Comparison of surface and core Si-Si bond lengths for the SiNWs with $100 \%$ coverage of $\mathrm{F}, \mathrm{H}$ and $\mathrm{OH}$. Yellow and black balls indicate the Si and passivating (F, $\mathrm{H}$ and $\mathrm{OH}$ ) atoms. SiNWs in top view are shown in line drawing for clarity to show the Si-Si bond lengths. (e) Work function of commonly used semiconductors with different doping levels vs. calculated work function for various passivation coverage of F, H, OH from ref [251]. (f) Minority-carrier lifetimes in samples after different surface treatments. C@SiNW is C/Si core/shell nanowire array after carbon MPECVD and PtNPs@C@SiNW is platinum nanoparticle (PtNP) decorated carbon/silicon core/shell nanowire arrays. a-b adapted from Ref. [247]. Copyright 2012 Society of Photo Optical Instrumentation Engineers. c-d adapted from Ref. [251]. Copyright Springer-Verlag 2010. f replotted from Ref. [252].

\subsubsection{Contact for carrier collection}

After the nanowires are grown and the junction is formed, contacts must be deposited to extract electrons and holes efficiently. The single-lying nanowire solar cells are mainly contacted using ebeam exposure or photolithography and metal evaporation. For the radial junction solar cell, multiple lithography and etch steps are required [34].

The top contact configuration is superior to the wrapped contact configuration for radial junction solar cell and is experimentally more feasible [35]. M. Saif Islam et al. reported ultra-low contact resistance of bridged Si nanowire via depositing Al contact pads [255]. A general approach to an air-bridged ohmic contact on extended arrays of vertically aligned SiNWs without any assistance of time-intensive and serial lithographic processes was demonstrated by Woo Lee et al. [256]. Their study establishes a direct correlation between the thermally induced reactions in the SiNW/metal-contact system [257]. Moreover, the authors found that Kirkendall voiding in silicon nanowires may cause contact failure at the highest annealing temperature of $850^{\circ} \mathrm{C}$. While vertical nanowire arrays sometimes lead to impaired performance due to loose contacts between electrodes and SiNW arrays, inclined SiNWs exhibit excellent anti-reflection properties and better electrical contact [112]. Practically, nanowires with different growth directions show varying electrical properties. For instance, while $\langle 110\rangle,\langle 111\rangle,\langle 100\rangle$ nanowire has a crossover point from indirect to direct band gap when the diameter approaches a few nanometer [258], the band structure for $<112>$ Si nanowires is such that the band gap remains indirect even at extremely small diameters [259]. This indirect band gap structure can lead to poor electrical properties. Therefore, Si <112> nanowires may be counterproductive for photonic applications.

Presently, top electrical contacts on vertical nanowire arrays are mainly formed by sputter deposition of metal on partially exposed tips of nanowires. However, the metal grid may detrimental to the device due to shadowing losses [93]. Therefore, the key consideration for the top contact must be transparent to allow light to penetrate while maintaining conductivity. Obtaining conformal metal finger grids or transparent conductor coatings on high-aspect-ratio nanowires is challenging and often requires a much thicker layer and more uniform deposition techniques involving sputtering or electrodeposition than in planar cells [219]. 


\subsubsection{Electrical properties impurities and defects in SiNWs}

In order to obtain cost-effective solar cells, mg-Si and Upgraded Metallurgical-Grade (umg-Si) must be used in SiNW solar cell fabrication rather than sg-Si. Porous SiNWs will be formed by MAC etching [260]. The introduced roughness affects the reflectance slightly but aggravate surface recombination [37]. A study suggests that the overall resistance of the porous silicon nanowires is rather large compared to that of solid counterparts with similar dimension and the starting doping concentration [186]. Therefore, electrical influence is the main consideration. These "dirty" silicon materials contain more impurities, especially shallow acceptors and donors ( $\mathrm{B}, \mathrm{Al}$ and $\mathrm{P}$ ). The minority carrier lifetime is $~ 1 / 10$ of the sg-Si [261]. In addition, the carrier mobility is reduced due to dopant compensation and the presence of boron gives rise to the formation of the boron-oxygen (BO) defect [262].

The advent of mg-Si facilitates researchers to achieving higher efficiency. Silicon solar cells based on umg-Si with efficiency above 20\% was reported [261]. Jae Young Kwon et al. demonstrate a thin film $(<20 \mu \mathrm{m})$ solar cell based on umg-Si with tapered nanowires atop achieves efficiency $\eta>$ $8 \%$. Based on MAC etching process, Xiaopeng $\mathrm{Li}$ et al. proposed route to use mg-Si and umg-Si as a starting material and upgrade their purities to solar grade while nanostructuring them [260]. To conquer the drawbacks of dirty Si, two methods are beneficial: the chemical and metallurgical processes that can be applied to purify mg-Si to sg-Si [263]; the abovementioned radial junction for SiNW solar cell [78].

In addition to impurities in SiNWs, high density of structural defects in silicon nanowires plays an important role in the formation of silicon nanowires and in the determination of the morphology of the nanowires [140]. The coupling between the substitution atom and the vacancy leads to a low formation energy and the substitution-vacancy pair works like a single vacancy [264]. These defects inevitably lower the minority carrier lifetime and carrier mobility. A simple route to annihilate defects in silicon nanowires is thermal annealing [265].

\subsection{Single Si nanowire solar cells}

The hybrid nanoarchitectures suffer from relatively low efficiency and poor stabilities when compared with the single nanowire devices [266]. Compared with mass nanowires on the substrate with complexity, single nanowire device is simple and shows exotic properties. It can be integrated on micron or nano scale circuits to generate electricity serving as driving source for nano-photoelectronic devices.

A novel front-crescent cross-sectional morphology single nanowire was proposed and the electrical simulation forecasted that the light-conversion efficiency can be up to $15.70 \%$, showing an enhancement ratio of $43.77 \%$ relative to the circular counterparts [267]. Bozhi Tian et al. show that individual silicon nanowire can serve as robust power sources to drive ultralow-power electronics and nanosystems [34]. The p-i-n silicon nanowires take advantage of core/shell structure, which enables the carriers to separate in the radial direction. Therefore, the carrier collection distance is much smaller without substantial bulk recombination. The performance of core/shell SiNW solar cells is comparable to mass NWs-based devices, achieving $\boldsymbol{J}_{\boldsymbol{s c}}=\sim 23.9 \mathrm{~mA} /$ $\mathrm{cm}^{2}$ (upper bound, excluding metal covered and exposed p-core areas) and $\boldsymbol{J}_{\mathrm{sc}}=\sim 16.0 \mathrm{~mA} / \mathrm{cm}^{2}$ 
(lower bound, including metal-covered and exposed p-core areas) with an efficiency of 3.4\%. After, the device is used to drive the nano device as nanoscale power source devices, such as nanowire pH sensor, AND logic gate circuit, illustrated in Fig. 20 (a) and (b). The single SiNW photovoltaic elements are able to generate $50-200 \mathrm{pW}$ per nanowire at 1-sun illumination. Additionally, it can be connected via series connection and parallel connection in order to satisfy nanoelectronic application, such as power matching and impedance matching.

Harry A. Atwater's group transferred the VLS-grown SiNW on the degenerately doped n-type Si wafers coated with $100 \mathrm{~nm}$ of $\mathrm{Si}_{3} \mathrm{~N}_{4}$ and then tested the properties of semiconductor nanowires [268]. Their research provides an instrumental method for measuring the properties of single nanowires. This cell yielded a $\boldsymbol{V}_{\boldsymbol{~ o c}}$ of $190 \mathrm{mV}, \boldsymbol{J}_{\mathrm{sc}}$ of $5.0 \mathrm{~mA} \mathrm{~cm}{ }^{-2}$, and a fill factor of 0.40 , which leads to an overall solar energy conversion efficiency of $0.46 \%$. Afterwards, scanning photocurrent microscopy (SPCM) was used to confirm the local photocarrier collection within nanowire devices. The photocurrent intensity was measured as a function of the near-field scanning optical microscope (NSOM) tip position. Finally, the confocal-mode photocurrent image was analyzed after removing the NSOM tip. Fig. 20 (c) shows that the charge collection properties of the nanowire which is investigated by SPCM images, demonstrating that negative photocurrent collection with a peak locates at the rectifying junction. Additionally, the current decayed exponentially away from junction under reverse and forward voltage biases with a constant decay rate. By confocal-mode SPCM measurement, the hole diffusion lengths, $L_{p}$, ranged from 2 to $4 \mu \mathrm{m}$ under different biases, relatively long diffusion length in single semiconductor nanowires, as shown in Fig. 20 (d). 
(a)

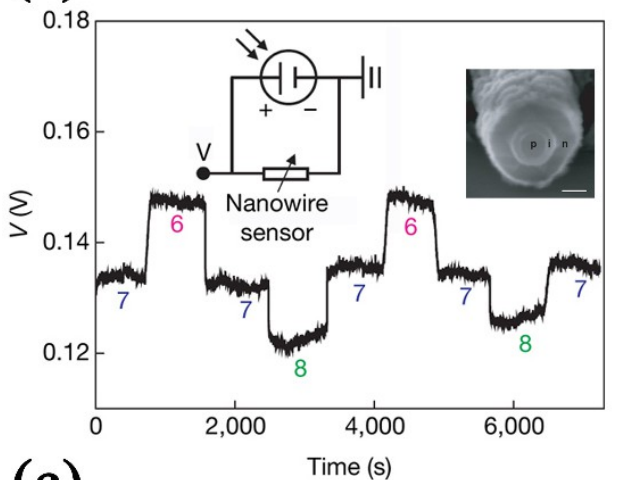

(c)

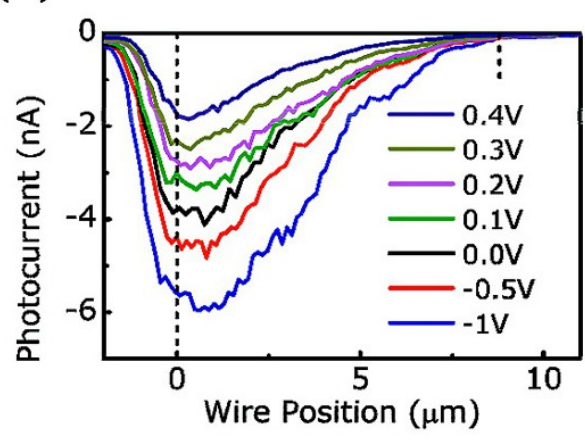

(b)

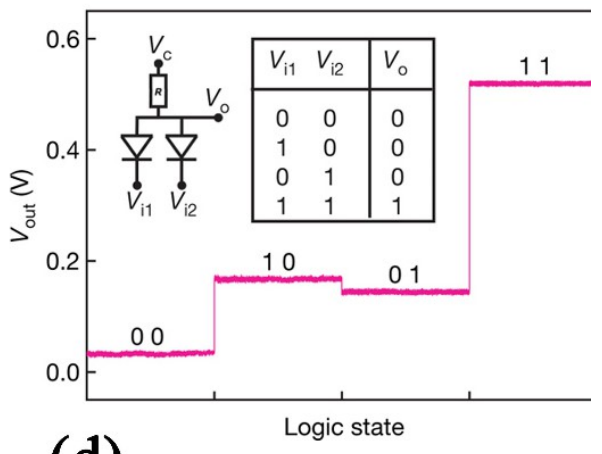

(d)

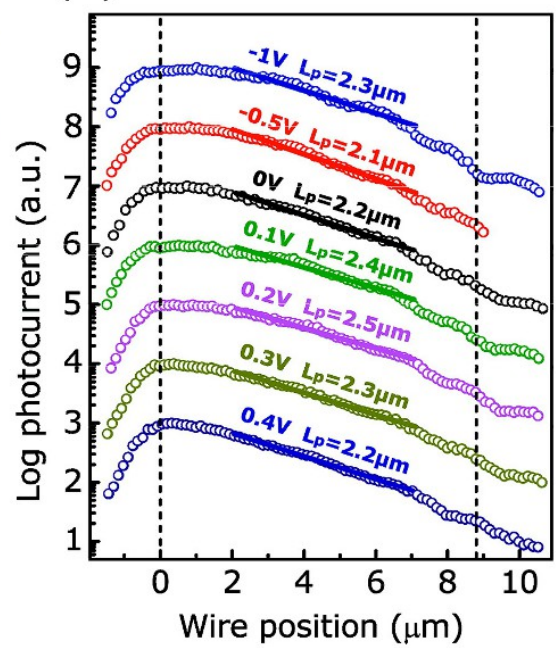

Figure 20 (a) Real-time detection of the voltage drop across an aminopropyltriethoxysilanemodified silicon nanowire at different $\mathrm{pH}$ values. The silicon nanowire $\mathrm{pH}$ sensor is powered by a single silicon nanowire photovoltaic device operating under 8-sun illumination $\left(\boldsymbol{V}_{o c}=0.34 \mathrm{~V}, \boldsymbol{I}_{s c}=\right.$ $8.75 \mathrm{nA}$ ). Inset, circuit schematics and SEM images of p-i-n core/shell single nanowire with a 100 nm scale bar. (b) Nanowire AND logic gate powered by two silicon nanowire photovoltaic devices in series. Insets, circuit schematics and truth table for the AND gate. The resistance of CdSe nanowire is $\sim 5 \mathrm{GV}$; the $\boldsymbol{V}_{\boldsymbol{~ o c}}$ of two photovoltaic devices in series is $0.53 \mathrm{~V}$. The large resistance of the CdSe nanowire and reverse-biased p-i-n diode makes $\mathrm{V}_{\mathrm{c}}$ and $\mathrm{V}_{\mathrm{i}}$ (HIGH) very close to $\boldsymbol{V}_{\boldsymbol{o c}}$ of the photovoltaic device. To get $\mathrm{V}_{\mathrm{i}}$ (LOW), the diode is simply grounded. (c) Line scans of confocal SPCM measurements obtained for several biases; (d) log-scale line scans of photocurrent as a function of the illumination position along the wire. The exponential fits enable extraction of the minority carrier (hole) diffusion length, $L_{p}$. The scans have been offset for clarity. In panels (c) and (d), the dashed vertical lines indicate the length of the wire with the junction located at $0 \mu \mathrm{m}$. a-b reprinted by permission from Macmillan Publishers Ltd: [34], copyright 2007. c-d reprinted with permission from. Ref. [268]. Copyright 2008 American Chemical Society.

In addition to be used as nano driving source [34, 49, 269-271], the single nanowire solar cells also simplify analysis of careful study of fundamental processes such as charge transfer, surface recombination [272]. They do not have complications like in the arrays that have many challenges including geometrical and practical limit on wire packing density. Therefore they show high 
efficiency beyond the Shockley-Queisser limit [273]. Despite these advantages, their disadvantageous aspects should be taken into account. Traditional transport measurements, such as hall effect measurements, secondary ion mass spectrometry (SIMS), Auger electron spectroscopy (AES), and X-ray photoelectron spectroscopy (XPS) are feeble to obtain meaningful results of single nanowire, but back-gated or top-gated field-effect transistor (FET) can be applied to measure its properties. Besides, expensive and low throughput e-beam lithography is commonly used for ohmic contact of single nanowire. Additionally, most of the reported single nanowire were not subject to vertical illumination because the detached wires were lying horizontally on the transferred substrate.

The crucial consideration for maximizing the efficiency of the single-wire radial junction solar cell is to augment the minority carrier diffusion length. Compared to the lying single nanowire, a standing nanowire is essentially finite in length. However, there is no quantitative analysis of the properties between the lying single nanowire and the standing nanowire.

\subsection{Plamonic SiNW solar cells}

The emerging field of plasmonics provides another light-trapping method to reduce the physical thickness of solar photovoltaic absorber layers while maintaining high efficiency. According to Atwater and Polman, there are mainly three ways to realize cost-effective solar cells via plasmonic structures [274]. First, metallic nanoparticle can be used as subwavelength scattering elements to couple the sunlight into substrate and thus enhancing optical path length. Second, metallic nanoparticle can be used as subwavelength antenna to couple near electric field into substrate. Finally, corrugated metallic film on the back surface of solar cells can offer surface plasmon polaritons (SPPs) supported at the metal/semiconductor interface and guided modes in semiconductor slab.

Optical simulation on NW solar cells decorated with plasmonic nanoparticles have been reported $[275,276]$. Si solar cell structure that combines the concept of nanowire decorated surface and a nanostructured Ag back reflector is simulated by FDTD simulation , as shown in Fig. 21 (a) [275]. Significant absorption enhancement is observed by patterning the back reflector with various periodical hemispherical Ag nanostructures above $500 \mathrm{~nm}$ because of the light scattering of $\mathrm{Ag}$ back reflector. Fig. 21 (b) plots the absorption spectrum with radius of hemispheres varies from 25 to $200 \mathrm{~nm}$. It can be seen that the absorption spectrum is sensitive to the radius of hemispheres in the large wavelength region. It can be explained by the change of localized surface plasmons (LSPs) resonance wavelengths, which is influenced by geometrical parameters of the plasmonic Ag hemispheres. Short circuit current densities were calculated according to data from in Fig. 21 (c). $\boldsymbol{J}_{s c}$ of the cell can be greatly improved by introducing optimal-sized hemisphere to the back reflector. A decline of $\boldsymbol{J}_{\boldsymbol{s c}}$ is observed as the radius increase because the LSPs resonance shifts to longer wavelengths where optical absorption loss dominates. With an optimal nanostructure radius of $50 \mathrm{~nm}, \boldsymbol{J}_{\mathrm{sc}}$ is improved to $27.8 \mathrm{~mA} / \mathrm{cm}^{2}$, which is $20 \%$ higher than that of a SiNW solar cell with planar a back reflector. Another model of adding $100 \mathrm{~nm} \mathrm{ZnO:Al} \mathrm{as} \mathrm{interlayer} \mathrm{in} \mathrm{the} \mathrm{cell,} \mathrm{as}$ illustrated in Fig. 21 (a) on the right. For the SiNW solar cell with a ZnO:Al/ hemisphere-Ag nanostructured back reflector, $\boldsymbol{J}_{\mathrm{sc}}$ is further increased to $28.4 \mathrm{~mA} / \mathrm{cm}^{2}$, as shown in Fig. 22 (d) and 
(e). Optical absorption loss in Ag nanoparticles is greatly suppressed via a $\mathrm{ZnO}$ :Al layer because the blueshift of dominated LSPs resonance at long wavelengths give rise to suppression of the LSPs induced parasitic absorption in Ag. Therefore, $\mathrm{ZnO}$ :Al/hemisphere-Ag nanostructure is an excellent light trapping scheme for SiNW solar cells.

(a)
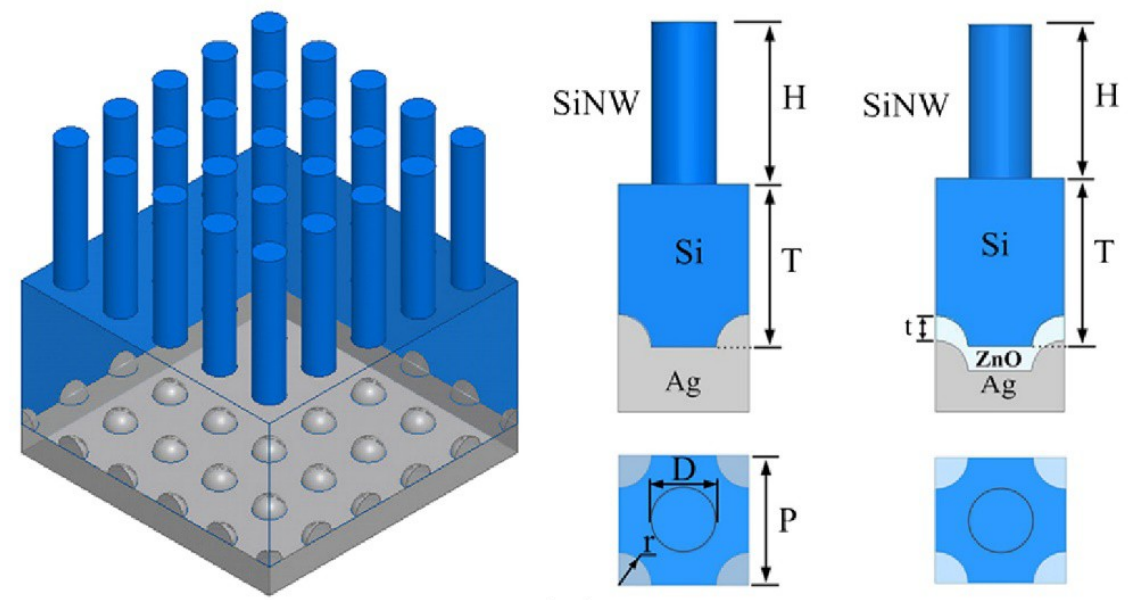

(b)

(c)

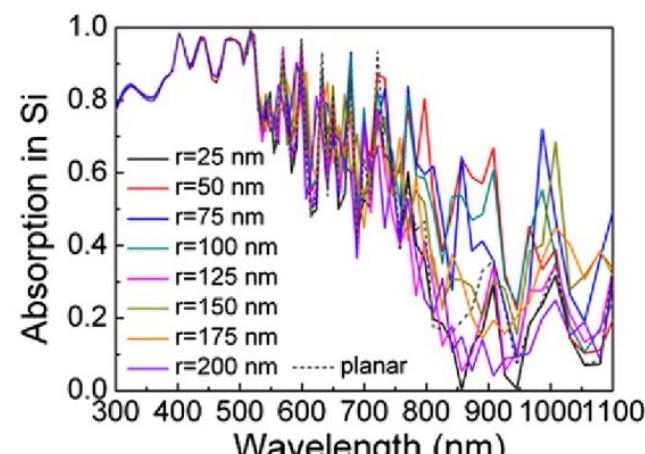

(d)

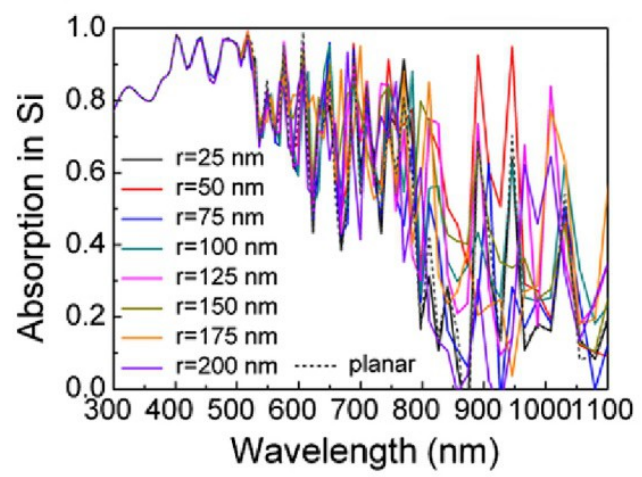

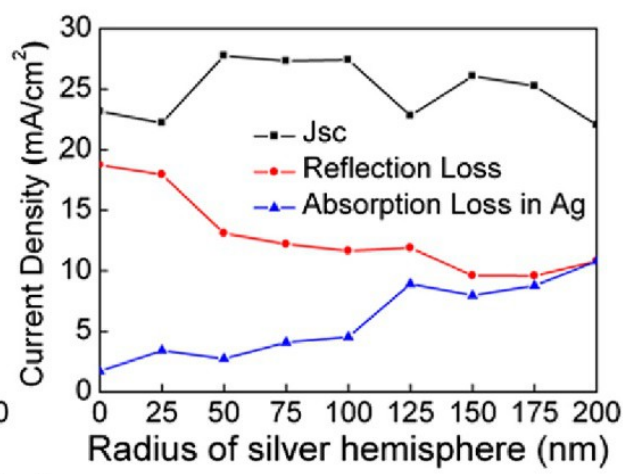

(e)

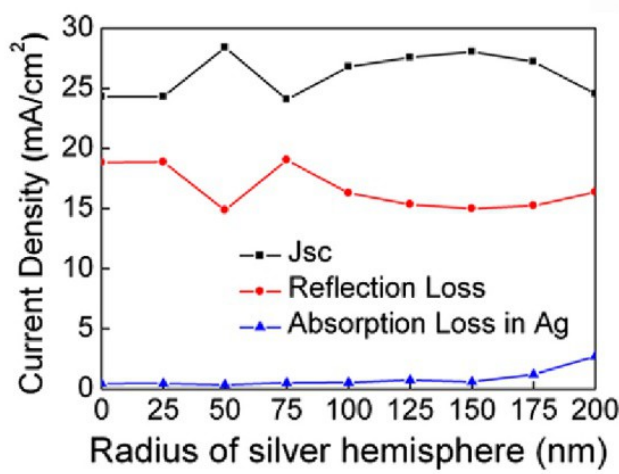

Figure 21 (a) Left: three-dimensional schematic diagram of the proposed SiNW solar cell. Cross sectional view and top view of unit cell consisting of; middle: a hemisphere-Ag back reflector or; left: a ZnO:Al/hemisphere-Ag back reflector. Structural parameters of the solar cell are nanowire diameter $(\mathrm{D})$, height $(\mathrm{H})$, periodicity $(\mathrm{P})$, substrate thickness $(\mathrm{T})$, radius of the hemispherical nanostructure (r) and ZnO:Al layer thickness (t). (b) Absorptance of the SiNW solar cell patterned with a periodical hemisphere-Ag (solid curve) and a planar (dash curve) back reflector, the radius 
of hemisphere is varied from 25 to $200 \mathrm{~nm}$. (c) Short circuit current density, current density loss by reflection, and $\mathrm{Ag}$ absorption as a function of the radius of hemisphere for the periodical hemisphere-Ag. (d) Absorptance of the SiNW solar cell patterned with a periodical $\mathrm{ZnO}: \mathrm{Al} /$ hemisphere-Ag back reflector, the radius of hemisphere is varied from 25 to $200 \mathrm{~nm}$. (e) Short circuit current density, current density loss by reflection and Ag absorption as a function of the radius of hemisphere for the periodical $\mathrm{ZnO}: \mathrm{Al} / \mathrm{hemisphere-Ag.} \mathrm{Reprinted} \mathrm{with} \mathrm{permission}$ from Ref. [275]. (c) 2012 Optical Society of America.

Keya Zhou et al. performed simulation on optical properties of SiNW array solar cells comparing the flat solar cell and nanohole-contact-grating layer (NCG) Ag back reflectors (BR) contacts, illustrated in Fig. 22 (a) [277]. As shown in Fig. 22 (b), highest energy loss in Ag BR was almost $\sim 14.5 \%$ for the SiNW array $(\mathrm{a}=100 \mathrm{~nm}, \mathrm{~d}=60 \mathrm{~nm}$ ) solar cells, while for the SiNW array with $\mathrm{a}=$ $500 \mathrm{~nm}, \mathrm{~d}=300 \mathrm{~nm}$, the energy loss was only $\sim 5 \%$. Despite the optical losses by the Ag BR, the enhanced near-field caused by the LSPs at the bottom of the SiNW compensates the loss and thus boosts the ultimate efficiencies of the SiNW solar cells after integrating them with a NCG Ag BR, where the optimal embedded wire depth was $\sim 20 \mathrm{~nm}$. Emre Mulazimoglu et al. deposit silver nanoparticles on vertical-arrayed SiNWs to obtain optical absorption enhancement [278]. Almost 50-fold enhanced LSPs was obtained for $24 \mathrm{~nm}$ thick silver deposited on $5 \mu \mathrm{m}$ long silicon nanowires and this can be ascribed to the modification in the dielectric constant of effective medium created by defect states, demonstrated in Fig. 22 (c). This work verified that the interaction of NWs and plasmonic source can be combined and harnessed to achieve perfect optical absorption for solar cells.

Although it is attractive to combine this two light trapping schemes for cost-effective solar cells, SiNWs array with plasmonic-particle decorated ruin the performance, sometimes even detrimental to the optical enhancement. Chenxi Lin studied hemispherical metal caps on SiNW array [279]. Fig. 22 (d) (left) shows the spectrum for a silver-capped array with $\boldsymbol{d}=\boldsymbol{a}=100 \mathrm{~nm}$. Contrary to works from others, this case corresponds to a low overall absorption ratio. Fig. 22 (d) (right) shows data for a silver-capped array with $\boldsymbol{a}=450 \mathrm{~nm}$ and $\boldsymbol{d}=100 \mathrm{~nm}$. The absorption for the capped array is lower than the bare array for low wavelengths. This phenomenon can be explained by two reasons: first, localized plasmon resonance of the cap occurs in the red/near-IR region, which does not offer enough enhancement to offset the lower absorption at short wavelengths; second, plasmonics, which couples light into guided modes, is not necessary because the NWs already provide this coupling. Their study gives an illustrative example of how plasmonic particle can degrade optical performance of devices. Therefore, the "plasmonic solution" is not universally valid. 

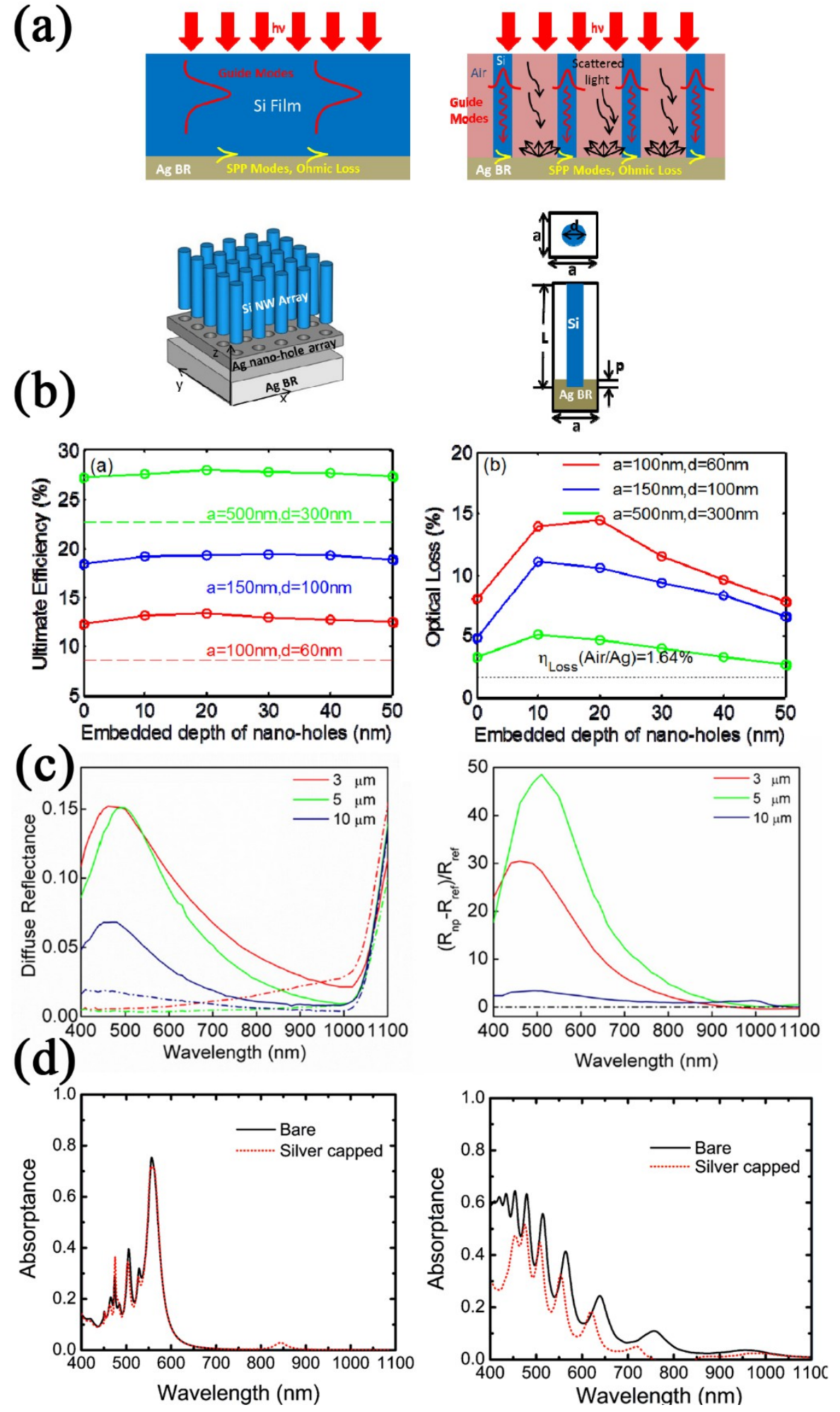

Figure 22 (a) Conceptual schematics showing light trapping and optical losses in Ag BR for simulation in Chenxi Lin's simulation, where d, l, a , and p correspond to the wire diameter, wire length, lattice constant, and embedded depth of NWs into the Ag BR, respectively. (b) The ultimate efficiencies calculated and optical losses using three different geometrical parameters are shown as a function of the embedded depth of Ag nanoholes. For reference, each dashed line (color-matched) denotes the ultimate efficiency value of bare SiNW arrays without a Ag BR.(c) Diffuse reflectance spectra and spectral enhancement ratios for $24 \mathrm{~nm}$ thick Ag deposited 3, 5, and 
$10 \mu \mathrm{m}$ long SiNWs. The spectral enhancement ratios is $\left(\mathrm{R}_{\mathrm{np}}-\mathrm{R}_{\mathrm{ref}}\right) / \mathrm{R}_{\mathrm{ref}}$, where $\mathrm{R}_{\mathrm{np}}$ is the reflectance of particle-decorated SiNW device. (d) The absorptance spectrum of bare (solid) and silver capped (dotted) SiNW arrays with $\mathrm{L}=500 \mathrm{~nm}, \mathrm{~d}=100 \mathrm{~nm}$, and a=100 nm (left) and $450 \mathrm{~nm}$ (right), where $\boldsymbol{L}$ is the length of NWs, $\boldsymbol{d}$ is the diameter of NWs and $\boldsymbol{a}$ is the period. a-b reprinted with permission from Ref. [277]. (c) 2012 Optical Society of America. c-d reprinted from Ref. [278] and [279] respectively with the permission of AIP Publishing.

There are only few reports regarding the plasmonic SiNWs solar cells until now. This integrated light trapping solution for cost-effective solar cells needs to be investigated via more details and it is quite promising for optoelectronic and photonic devices. As two approach to reduce material use for energy harvesting, the benefits of plasmonic decorated SiNWs can further cut the material consumption. Additionally, for bulk recombination-dominated Si material, thin solar cells also present the advantage of better carrier collection and reduced bulk recombination, both factors improving the solar cell efficiency. As a result, an ultra-thin device can be obtained with high performance. However, the implementation of this hybrid high-efficiency devices that can be manufactured at a competitive price is so far mainly limited by the fact that the fields are still relatively new. There are two challenges: first, scattering objects with high effective cross section over the available solar spectrum need to be realized; second, the coupling method for incorporating plasmonic source on the dense nanowires.

\subsection{Hybrid SiNW solar cells}

Conventional $\mathrm{p}^{-\mathrm{n}}$ junction-based solar cells have $\mathrm{p}$ and $\mathrm{n}$ region to separate and extract lightgenerated charge carriers. However, the $\mathrm{p}$ and $\mathrm{n}$ doping processes is conventionally formed by ion implantation and annealing processes or dopant diffusion processes which are extremely expensive and demanding condition that requres high temperatures approximately $1000{ }^{\circ} \mathrm{C}$. Therefore, it is intriguing to form a heterojunction at low temperatures. One of the solutions is to combine an organic material with silicon. As a substitute for expensive homojunction of $\mathrm{Si}$, the Schottky junction formed by depositing organic materials via a solution-processed method is costeffective. So far, the organic/inorganic solar cell based on hybrid composites of conjugated molecules and SiNW on a planar substrate yielded an excellent power conversion efficiency (PCE) above 10\% [242, 280-283].

Xiaojuan Shen et al. demonstrated that both the SiNWs structure and the thin organic layer top contact were critical to achieve a high performance organic/silicon solar cell [280]. The organic semiconductor, 2,2,7,7'-tetrakis(N,N-di-p-methoxyphenyl-amine)-9,9'-spirobifluorene (spiroOMeTAD), was coated on SiNWs standing on a planar substrate, as shown in Fig. 23. The thin spiro-OMeTAD layer gives rise to considerable charge properties and thus causes a high EQE value. The device 1 and 2 presented a large EQE value of $90 \%$ at their peaks over 300-1100 nm, comparable with traditional silicon solar cells fabricated at high temperature. However, the control group, device 3, presented a dwarf EQE value at its peak of $54 \%$. This can be ascribed to the inefficient hole collection efficiency in the long-distance transport process from the interface to the top electrode [280]. For comparison, the device 3 showed inferior performance with a $\boldsymbol{V}_{\boldsymbol{o c}}$ of $0.504 \mathrm{~V}$, a $\boldsymbol{J}_{s c}$ of $16.7 \mathrm{~mA} / \mathrm{cm}^{2}$, and a $F F$ of 0.370 for an overall estimated PCE of 3.1\%. The poor performance of this device can be attributed to lower hole transport efficiency. Other studies 
complete this field. Hong-Jhang Syu's work suggests that long SiNWs have better light absorption, but coverage of PEDOT:PSS over the SiNWs needs to be improved and proper passivation on the surface of SiNWs is necessary for better performance of SiNWs/PEDOT:PSS solar cells [284]. Ching-Fuh Lin's group fabricated SiNW/P3HT:PCBM blend hybrid solar cells using the SiNW transfer technique without any vacuum equipment or high-temperature processing, indicating that it can be applied to plastic solar cells because it can be processed fast, cheaply and simple [192]. By combination with a back-surface distribution of silver nanoparticles, Manisha Sharma et al. experimentally observed a promising light-trapping scheme in c-Si heterojunction hybrid solar cell of $5.7 \mu \mathrm{m}$ thickness with a topography that included a SiNW array used in conjunction with the organic semiconductor PEDOT:PSS [285]. Although this hybrid structure allows facile process, interface engineering is an essential consideration to achieve high efficiency solar cells. 13.1\% efficiency hybrid organic/silicon-nanowire heterojunction solar cell via modified organic surface morphology was reported [281]. Afterwards, a transfer-imprinted $\mathrm{Au}$ mesh electrode was used and a 13.2\% efficiency Si nanowire/PEDOT:PSS hybrid solar cell was reported [286]. A solution process was adopted to overcome poor coverage of organic material PEDOT:PSS and a efficiency of $13.36 \%$ was achieved [287].

(a)

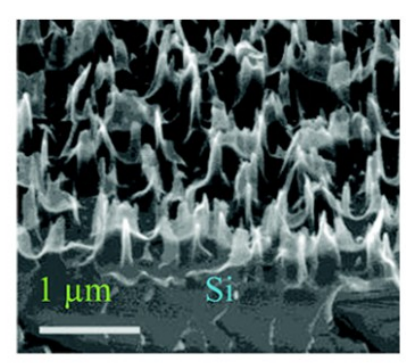

(c)

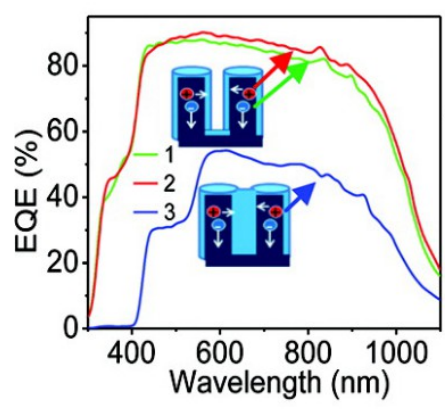

(b)

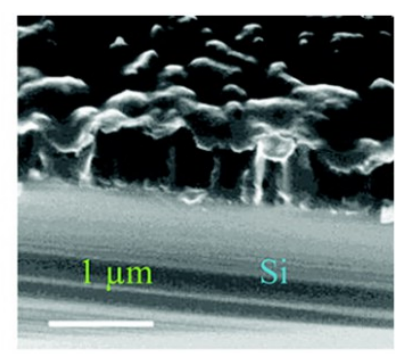

(d)

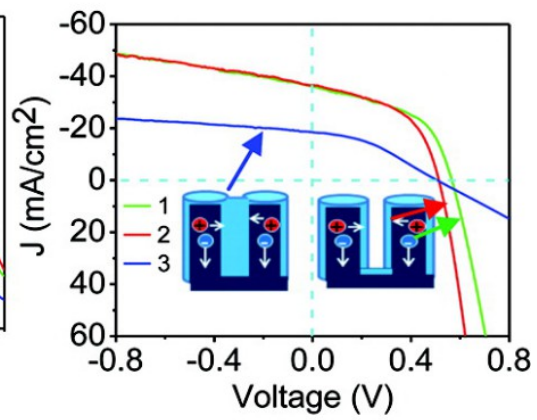

Figure 23 (a) Cross-section SEM image of In:Ga/SiNWs/spiro-OMe-TAD/PEDOT:PSS/Cu structure with the core/shell structure; (b) Cross-section SEM micrograph of In:Ga/SiNWs/spiroOMeTAD/Cu structure with the embedded structure; (c) EQE spectra and $\boldsymbol{J}-\boldsymbol{V}$ curves of device 1 (In:Ga/SiNWs/spiro-OMe-TAD/PEDOT:PSS/Cu (core/shell)), device 2 (In:Ga/SiNWs/spiroOMeTAD/Cu (core/shell)), and device 3 (In:Ga/SiNWs/spiro-OMe-TAD/Cu (embedded structure)). Reprinted with permission from Ref. [280]. Copyright 2011 American Chemical Society.

However the space among dense SiNWs is usually too small. On one hand, large density of nanowires increase absorption, on the other hand, the space between NWs are too small to be 
filled with by big conjugated polymers. The alkali-treated process provides superior coverage of PEDOT:PSS down to the bottom of the NWs [288]. Small organic molecules, such as Tris(8hydroxyquinolinato) aluminium ( $\left.\mathrm{Alq}_{3}\right)$ and 1,3-bis(2-(4-tert-butylphenyl)-1,3,4-oxadiazol-5-yl) benzene (OXD-7) can be applied to fill the narrow gap [289]. Nanocones [93], tapered NWs [53] and nanotubes [283, 290,291] are also introduced to overcome this problem. For instance, a novel method was adopted to fabricate 3D n-SiNW heterojunction solar cells incorporated with functionalized mlulti-walled nanotubes (MWNTs) by Gopal Kalita et al [290]. The free standing SiNWs are fabricated with electroless etching process, followed by combining MWNTs along with the polymer. The device structure is shown in Fig. 24 (a) and (b), composed with n-SiNWs and poly (3-octylthiophene) (P3OT) incorporating carbon nanotubes (CNTs). Fig. 24 (c) and (d) present $\boldsymbol{I}-\boldsymbol{V}$ characteristics under dark and white light illumination of the device fabricated with the structure P3OT/n-SiNWs and P3OT + O-MWNTs/n-SiNWs. The P3OT/n- Si structure displayed poor performance with $\boldsymbol{\eta}=0.0079 \%$ and P3OT/n-Si NWs structure presents PCE $0.19 \%$, respectively. P3OT + O-MWNTs/n-SiNWs shows a PCE with $0.61 \%$. It can be concluded that the P3OP/n- Si NWs structure is more efficient in electron collection and transportation in the active layer, indicating that although there is restricted hole conduction in the 3D structure due to the poor mobility of the conducting polymer, efficient electron collection and transportation can be achieved by the P3OP/n- SiNWs structure by improving photoexciton dissociation and electron transportation. The significantly improved efficiency of incorporation of oxygen plasma treated MWNTs (O-MWNTs) is due to the reduced recombination loss. Therefore, this approach offers a route to fabricate doped SiNWs solar cells along with conducting polymers and carbon nanotubes.

(a)

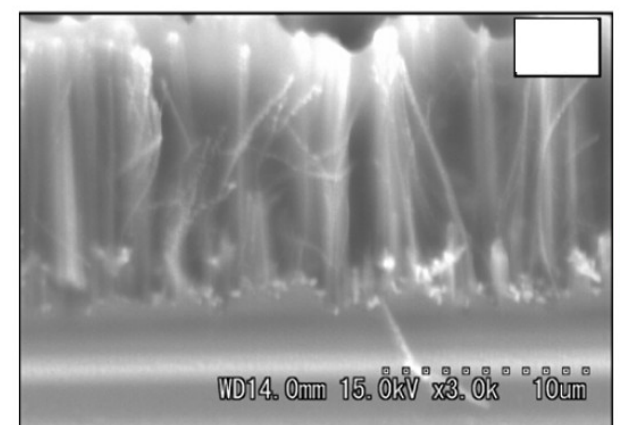

(c)

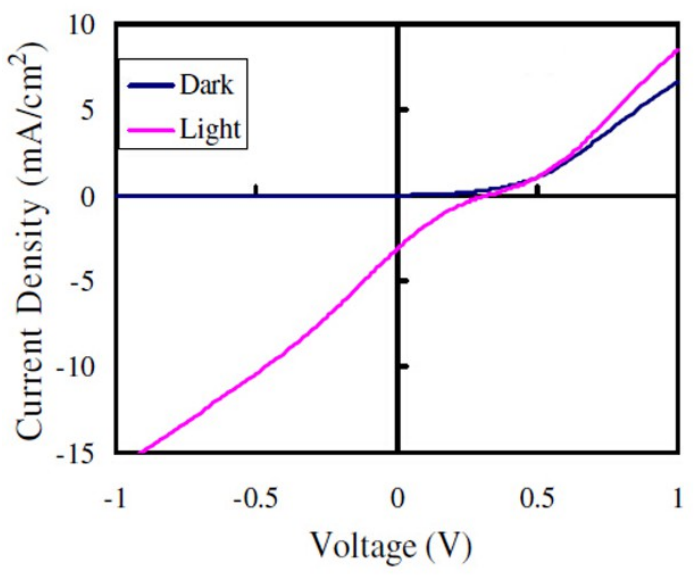

(b)

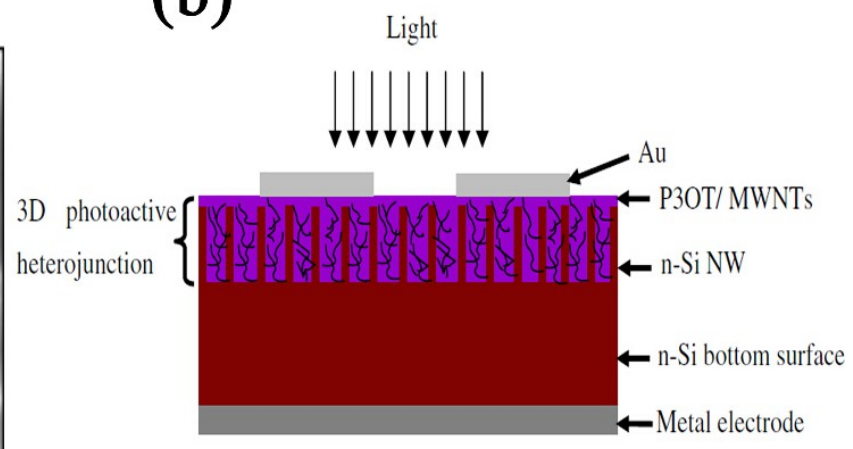

(d)

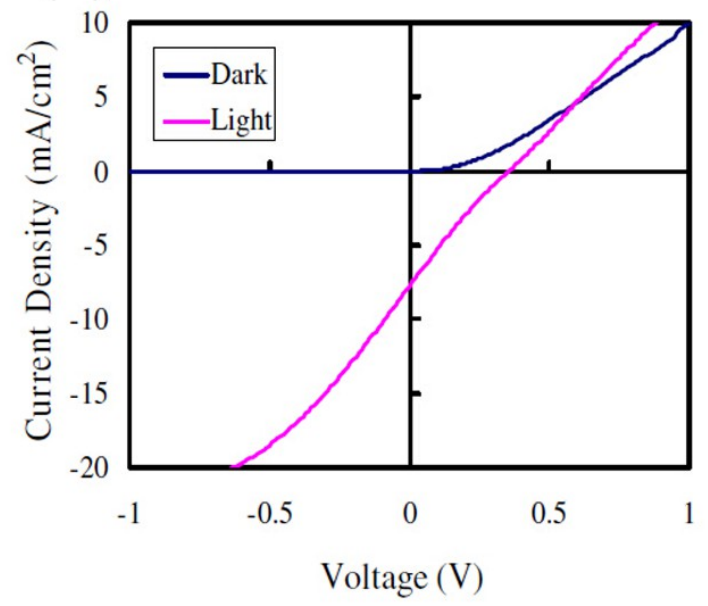

Figure 24 (a) SEM image of interpenetrating polymer and NWs composite film, NWs vertical 
alignment remain undisturbed after the polymer casting; (b) Schematic diagram of the fabricated hybrid solar cell with n-Si NWs and P3OT incorporating CNTs; (c) and (d) $I-V$ characteristics of the device fabricated with the structure (c) P3OT/n-Si NWs and (d) P3OT + O-MWNTs/n-Si NWs with the incorporation of CNTs under dark and illumination (AM $1.5100 \mathrm{~mW} \mathrm{~cm}^{-2}$ ), where OMWNTs is the oxygen plasma treated MWNTs. Adapted from Ref. [290]. Copyright 2009 IOP Publishing.

According to the abovementioned studies, the choice of organic materials need to satisfy two factors: being transparent and conductive, which ensure the most incoming light absorption. The most commonly used materials are the conjugated polymers, such as PEDOT:PSS, poly. However, such materials have large molecules, which are prone to aggregate at the top because of the narrow cavities between the NWs, leading to a low fill factor and a higher chance of carrier recombination and thus a lower PCE. Attempts need to be done toward two directions: first, smaller conjugated molecule need to be found while being transparent and conductive; second, exotic structures need to be investigated to be compatible with these commonly-used materials.

Apart from the hybrid inorganic-organic solar cell, another method is adopted to make a device composed of a single-crystalline Si wafer surrounded by ultrathin amorphous Si layers, which is called a heterojunction with intrinsic thin layer (HIT) solar cell. HIT-nanowire-based solar cells attract more attention for suppressed surface recombination in the c-Si. The HIT core-shell nanowire based structure is mainly composed of core c-SiNWs and shell hydrogenated amorphous silicon (a-Si:H) wrapping. In this structure, the a-Si:H that can be simply deposited by plasma enhanced chemical vapor deposition (PECVD) offering excellent passivation of the silicon surface.

Extensive investigations show that the HIT-nanowire-based solar cells is a low-cost process for solar cell preparation resulting in a considerable efficiency [242, 292-295]. The carbon nanotube membranes is introduced as wrapping layer to substitute a-Si:H [296]. Guobin Jia et al. proposed a novel multiple core-shell TCO/ $\mathrm{Al}_{2} \mathrm{O}_{3} / \mathrm{a}-\mathrm{Si}: \mathrm{H}\left(\mathrm{p}^{+}+\mathrm{i}\right) / \mathrm{c}-\mathrm{SiNW}$ structure as shown in Fig. 25 (a), (b) and (c). EQE spectra and $\boldsymbol{J}-\boldsymbol{V}$ curve reveal that the solar cell with ultrathin $\mathrm{Al}_{2} \mathrm{O}_{3}$ exhibits higher EQE almost over the whole range of the spectrum than that of the $\mathrm{Al}_{2} \mathrm{O}_{3}$ absent one. By optimization of the a-Si:H emitter, an efficiency of $10.04 \%$ has been reached on the multiple core-shell nanowires based solar cells, shown in Fig. 25 (d). The negatively charged $\mathrm{Al}_{2} \mathrm{O}_{3}$ will repulse the minority carriers away from the surface, which effectively reduces the recombination at the surface of the a-Si:H emitter [295]. Owing to the existence of the ultrathin $\mathrm{Al}_{2} \mathrm{O}_{3}$, a much lower saturation current is observed from the measured data of $\boldsymbol{J}_{\boldsymbol{s c}}$ and $\boldsymbol{V}_{\boldsymbol{o c}}$. As a result, the the effective minority carrier diffusion length in the a-Si:H emitter is elongated. In this structure, the absorption in the amorphous Si layer reduces the intensity of the current generated by the devices because of strong absorption losses. By optimizing the deposition conditions of amorphous layer, a $12.9 \%$ efficiency was reported [297]. 


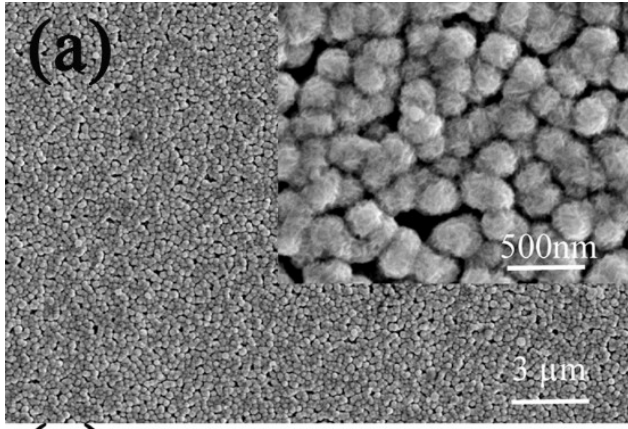

(c)

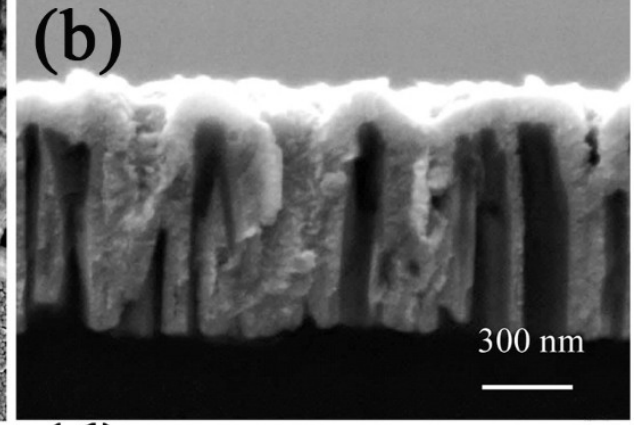

(d)
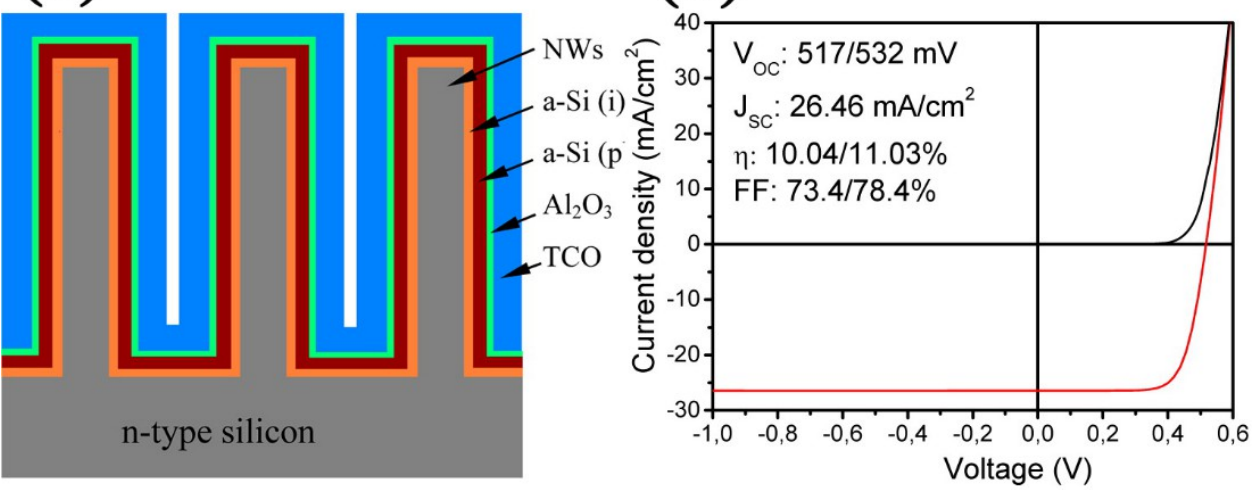

Figure 25 (a) Top view SEM image of the nanowire based solar cells (inset, high-resolution SEM image); (b) SEM cross-section image; (c) Sketch of the multiple core-shell nanowire based solar cell on SiNW arrays; (d) $\boldsymbol{J}$ - $\boldsymbol{V}$ curves measured in the dark (black line) and under AM 1.5 (red line) of the solar cell with $10.04 \%$ efficiency. The second values in the inset are determined by Suns- $\boldsymbol{V}_{\text {oc }}$ measurements Reprinted with permission from Ref. [295]. Copyright 2013 American Chemical Society.

To make a high performance HIT nanowire-based solar cell, the cleaned c-Si surface is essential. However, the introduction of impurities during densely packed nanowire fabrication is unavoidable i.e. metal catalyst used during the procedure. This would pose a threat to high performance devices, presenting contaminations at the junction interface and influencing the performance dramatically due to recombination. Procedure for removal of metal contamination is urgent. For example, a three-step cleaning procedure was developed to remove the silver contamination [292]. As a result, a great improvement of $\boldsymbol{J}-\boldsymbol{V}$ characteristics and $\boldsymbol{V}_{\text {oc }}$ was achieved in comparison with the unclean one. Meanwhile, light absorption in the UV to blue spectral region in the aluminum doped zinc oxide (AZO) layer (As transparent conductive oxide) is another issue. These issues can be addressed by tuning the band gap of the AZO layer to obtain maximum transparency or by using deep UV transparent contact materials like grapheme [293], Ga-doped $\mathrm{ZnO}$ [298] or $\beta-\mathrm{Ga}_{2} \mathrm{O}_{3}$ [299] layers as transparent contact. Additionally, sulfur-assisted growth of Si nanowires by VLS method was regarded as the best candidates for unique photovoltaic application. Another method to address this issue is surface passivation.

In this part, hybrid SiNW solar cells are reviewed. So far, the record efficiency of this cell has been raised above $10 \%$. It is most approaching method for cost-effective solar cells for their less demanding fabrication condition at moderate temperature with less energy consumption. The 
material choice in the hybrid cells design for practical option should be lower cost Si thin films rather than using expensive bulk Si wafer. The inorganic/organic heterojunction solar cells are advantageous over conventional $\mathrm{p}-\mathrm{n}$ junction solar cells due to their low fabrication cost including low thermal budget, simple, rapid and solution based processes during heterojunction fabrication. However, the device deteriorate when exposed to atmosphere with a normal content of oxygen and humidity level [300]. Presently, encapsulation is the only solution for large-scale organic/inorganic solar cell production but it complicates the fabrication processes and increases the production cost. Further study should focus on stability. Water adhesion in the process of PEDOT:PSS deposition dramatically affects the quality of the heterojunctions between SiNWs and PEDOT:PSS [301]. Eliminating the water trapping has tangible effect on improving the stability of SiNWs/PEDOT:PSS hybrid solar cells in the environment humidity.

\subsection{SiNW Photoelectrochemical solar Cells}

Photoelectrochemical (PEC) cells are solar cells that generate electrical energy or hydrogen in a process similar to the electrolysis of water and they offer a convenient and facile contact with NWs via immersing the substrates into a liquid electrolyte. In these PEC solar cells, SiNWs are used as photoelectrodes. The SiNWs can be applied not only on water splitting for hydrogen production but also on solar energy conversion. The semiconductor/liquid junction architecture cells provide potential cost reduction over their solid-state counterparts due to less material consumption.

Extensive methods have focused on achieving this inexpensive cell [252, 302-310]. Kuiqing Peng et al. fabricate SiNWs with suppressed optical reflectance on n-Si (100) substrate, as shown in Fig. 26 (a) and (b). Reflectance of SiNWs prepared in the $\mathrm{HF}-\mathrm{AgNO}_{3}$ and $\mathrm{Ag}-\mathrm{HF}-\mathrm{H}_{2} \mathrm{O}_{2}$ solution are compared with porous $\mathrm{Si}$ and polished Si, presented in Fig. 26 (c). The SiNW arrays with rough surface prepared by $\mathrm{HF}-\mathrm{AgNO}_{3}$ and $\mathrm{Ag}-\mathrm{HF}-\mathrm{H}_{2} \mathrm{O}_{2}$ have minimum reflection making them a promising photoactive PEC material. Fig. 26 (d) illustrates the measurement schematic of the photovoltaic PEC solar cells. Fig. 26 (e) shows the photovoltaic IV curve of four compared samples: The $\boldsymbol{V}_{\boldsymbol{o c}}$ of $\mathrm{HF}-\mathrm{AgNO}_{3}$ is significantly increased, from 0.43 to $0.73 \mathrm{~V}$, compared with clean polished n-Si wafer. This mirrors low SRV and good bulk properties of SiNWs. Since SiNWs minimize the reflectance, the photogenerated current of SiNW photoelectrodes is boosted, from 0.07 to $0.872 \mathrm{~mA} / \mathrm{cm}^{2}$. 


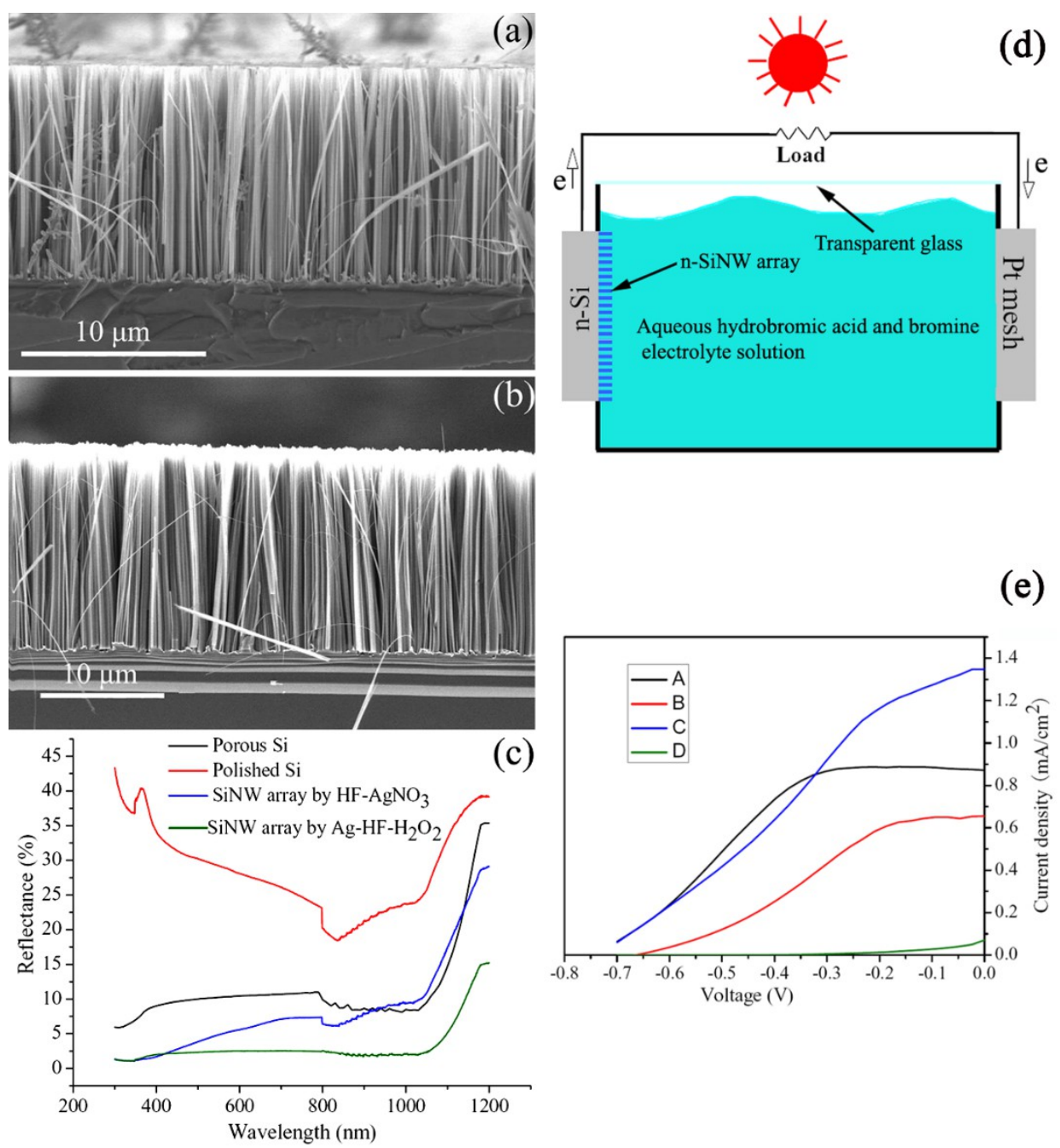

Figure 26 (a) Typical cross-sectional SEM image of SiNW array produced on 7-13 $\Omega \mathrm{cm} n$-Si (100) substrate in $5 \mathrm{M} \mathrm{HF} / 0.02 \mathrm{M} \mathrm{AgNO}_{3}$ solution. Silver dendrites can be observed on and within the SiNW array. (b) Typical cross-sectional SEM image of SiNW array produced on 7-13 $\Omega \mathrm{cm} \mathrm{n}$ $\mathrm{Si}$ (100) substrate in $10 \% \mathrm{HF} / 0.6 \% \mathrm{H}_{2} \mathrm{O}_{2}$ solution. (c) Hemispherical optical reflectance of SiNW arrays, porous silicon, and polished Si. (d) Schematic illustration of the photovoltaic PEC solar cell based on rough n-SiNW array. (e) Photocurrent densities-voltage curves of PEC solar cells

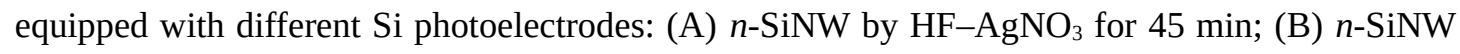
in Ag-HF- $\mathrm{H}_{2} \mathrm{O}_{2}$ for $45 \mathrm{~min}$; (C) $n$-SiNW in Ag-HF- $\mathrm{H}_{2} \mathrm{O}_{2}$ for 45 min without removal of residual back silver, and (D) clean polished $n$-Si wafer. Reprinted from Ref. [310] with the permission of AIP Publishing.

Nanoparticle-decorated SiNW arrays are introduced as photocathode [302, 311]. Kui-Qing Peng et al. decorated-type SiNW sidewalls with discrete 5-10 nm platinum nanoparticles and the PEC solar cells yielded PCE up to $8.14 \%$ by immersing into a liquid electrolyte containing $\mathrm{Br}^{-} / \mathrm{Br}_{2}$ redox couple [302]. By optimizing of Pt nanoparticles, the highest efficiency have obtained. Another group exhibits a Pt/SiNW photocathode which has a dramatically enhanced voltage of $0.42 \mathrm{~V}$, superior to that of a planar Si [311]. This enhancement can be ascribed to high surface roughness of the NWs, positive shift in $\mathrm{E}_{\mathrm{FB}}$ (flat-band potential) and light trapping of SiNWs. The optically transparent PDMS is used to fabricate the flexible, freestanding, polymer-supported 
SiNWs [303]. After peel-off from Si substrate, the peeled wire/polymer composite photoelectrodes demonstrate an efficiency above 5\% without improving the other parameters of these polymersupported wire array photoelectrodes. Uniform $\mathrm{TiO}_{2}$ nanorods were grown on dense $20 \mu \mathrm{m}$ long wet-etched SiNW backbones [304]. This 3D high-density hierarchical nanowire architecture yields a photoelectrochemical efficiency of $2.1 \%$, three times higher than that of $\mathrm{TiO}_{2}$ film-SiNWs with a core/shell structure. This study suggests that the 3D NW structure is likely superior to free standing NW arrays for PEC electrode design.

Although many experiments achieve promising results to some extent, PECs are still suffering from severe junction recombination and poor carrier collection and thus lead to low performances. Hence, reducing recombination losses and photocorrosion by surface passivation are critical for high-performance PECs. $\mathrm{TiO}_{2}$ [312, 313], $\mathrm{Ni}$ [314], $\mathrm{NiO}_{\mathrm{x}}$ [315-317], SiC coatings [318] are often used as inert to mitigate corrosion and oxidation for PECs. Hongjie Dai's group passivated silicon photoanodes by depositing a $\sim 2$ nanometer thick nickel film which offers high stability in aqueous $\mathrm{KOH}(\mathrm{pH}=14)$ and borate buffer $(\mathrm{pH}=9.5)$ [314]. The Ni-coated electrode showed a flat PEC water oxidation in a mixed lithium borate-potassium borate electrolyte during 80 test hours, as shown in Fig. 27 (a). Nathan S. Lewis's group exhibited $\mathrm{TiO}_{2}$ coatings prevent the photoanodes from corrosion [312]. A $44 \mathrm{~nm} \mathrm{TiO}_{2}$ on the n-p+-Si photoanode, in conjunction with $\mathrm{Ni}$ islands, decreased only by $\sim 10 \%$ after 100 hours of continuous operation, illustrated in Fig. 27 (b). However, this technique is only demonstrated on bulk Si photoanode. It is difficult to cover an ultra-thin film uniformly on the highly dense nanowire surface.

High-performace SiNW array PEC solar cells were demonstrated via surface passivation and modification [252]. The properties of heterostructured PEC solar cells exhibited PCE of 10.8\% and excellent stability even in the aggressive aqueous $\mathrm{HBr} / \mathrm{Br}_{2}$ electrolyte. While the photocurrent of naked H-SiNW drops to nearly zero after 4 hours illumination, the PtNPs@C@SiNWs showed less than $28 \%$ reduction in photocurrent after 10 hours of illumination, as shown in Fig. 27(c). Although, the cost-effective PEC solar cells accompanying with $\mathrm{HBr} / \mathrm{Br}_{2}$ electrolyte are potential for inexpensive cells, the aqueous $\mathrm{HBr} / \mathrm{Br}_{2}$ electrolyte is toxic, corrosive and volatile, and susceptible to water. Methyl viologen $2^{+/+}$are commonly used as substitute but it suffers from serious stability issues. Room temperature ionic liquid (IL), a mild molecular solvents to substitute the undesirable electrolyte, acts as electrolyte solvents in PEC solar cells, achieving efficiency of 6.0\% and allowing SiNWs to preserve advantageous stability [305]. In Fig. 27 (d), the SiNWs electrode demonstrates a very poor stability performance when an aqueous electrolyte is utilized even for the $-\mathrm{CH}_{3}$ terminated electrode. However, in the IL electrolyte solvent, then the methylated electrode yields a stability under 24 hours simulated AM 1.5 solar spectrum irradiation. 

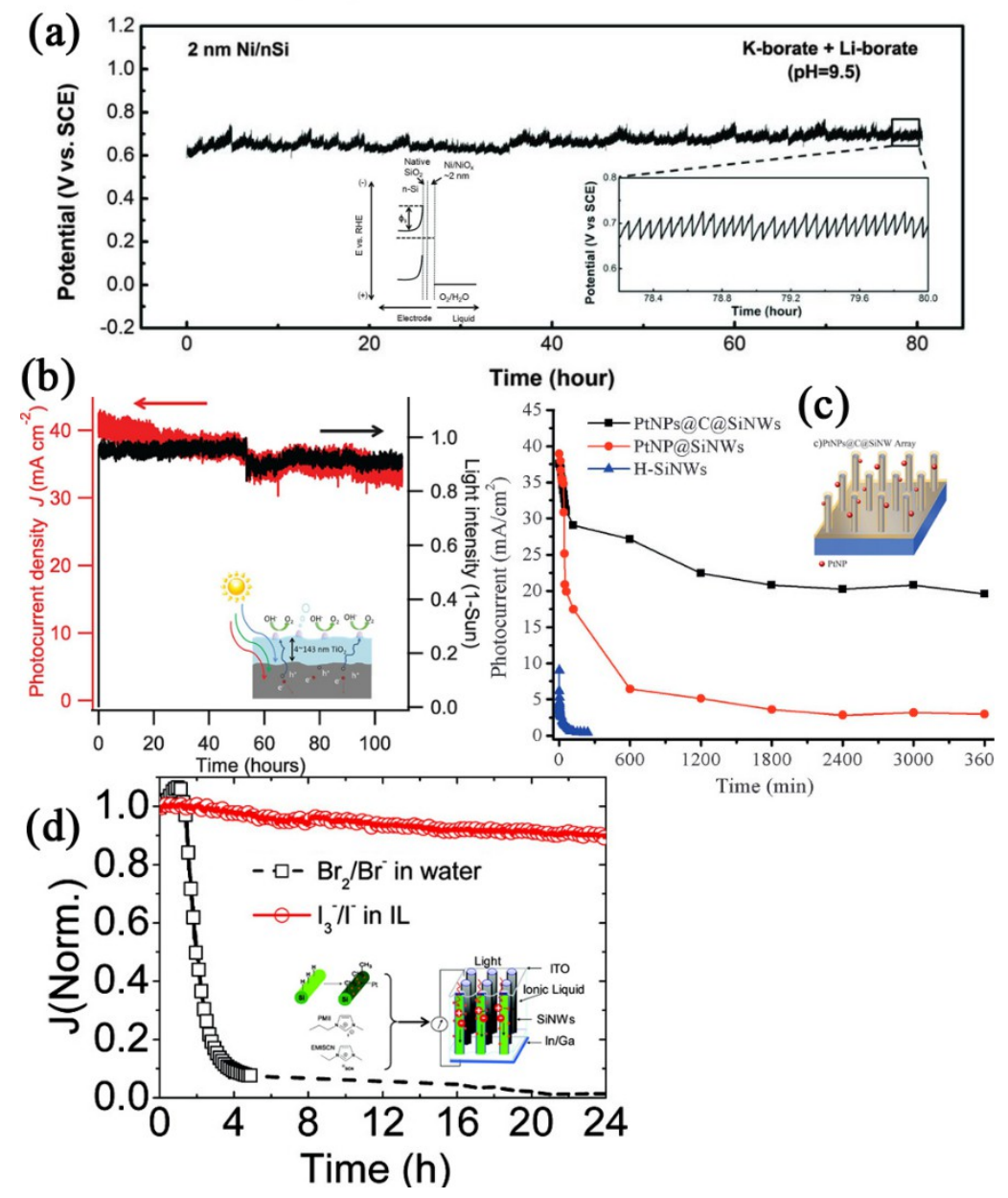

Figure 27 (a) Potential versus time data, under constant current density of $10 \mathrm{~mA} / \mathrm{cm}^{2}$, of a 2-nm $\mathrm{Ni} / \mathrm{n}$-Si anode in $0.65 \mathrm{M} \mathrm{K}$-borate and $0.35 \mathrm{M} \mathrm{Li}$-borate $(\mathrm{pH}=9.5)$ for 80 hours ( $>3$ days) under constant illumination. No PEC activity decay was observed. The left inset is the proposed approximate energy band diagram. The ultrathin $\mathrm{Ni} / \mathrm{NiO}_{\mathrm{x}}$ and the electrolyte form an effective layer interfacing with $\mathrm{Si}$, affording a high built-in potential $\Phi_{s}$ and photovoltage; the right Inset shows details of current fluctuations caused by oxygen gas bubble release. The increased saturation current in the 5-nm Ni-coated electrodes is highly reproducible, but the cause is currently unknown. (b) Chronoamperometry of an n-p+-Si photoanode coated with $44 \mathrm{~nm}$ of $\mathrm{TiO}_{2}$ and $\mathrm{Ni}$ islands for over 100 hours in $1.0 \mathrm{M} \mathrm{KOH}(\mathrm{aq})$. The photocurrent density versus time (red curve) was overlaid with the illumination intensity versus time (black curve). The electrode potential was held at $0.93 \mathrm{~V}$ versus saturated calomel electrode (SCE). The ENH-type tungstenhalogen light bulb burned out at the 54th hour and was then replaced with another ENH bulb, and the experiment was continued as indicated. The illumination intensity was 1.25 Sun. The inset is the cross-sectional schematic of a photoanode stabilized against corrosion in a $1.0 \mathrm{M} \mathrm{KOH}$ (aq) by a thick electronically defective layer of unannealed $\mathrm{TiO}_{2}$ deposited by ALD. Instead of corroding the anode, the photogenerated holes are conducted through the $\mathrm{TiO}_{2}$ to Ni electrocatalysts, where the holes are used to oxidize water to $\mathrm{O}_{2}$. (c) Photocurrent densities of H-SiNWs, PtNPs@SiNWs, and PtNPs@C@SiNWs as a function of measurement time; the inset is PtNPs@C@SiNW array illustration. (d) Normalized $\boldsymbol{J}_{\boldsymbol{s c}}$ versus time response of $-\mathrm{CH}_{3}$ SiNWs electrode under simulated AM 1.5 solar spectrum irradiation at $100 \mathrm{~mW} \mathrm{~cm}^{-2}$ using different electrolytes; $\mathrm{I}_{3}^{-} / \mathrm{I}^{-}$in IL liquid 
(solid line) and $\mathrm{Br}_{2} / \mathrm{Br}^{-}$in water (dash line). The inset is the schematic drawing of the PEC cell structure using SiNWs as electrode. a and b adapted from Ref. [314] and [312] respectively. Reprinted with permission from AAAS. c adapted from Ref. [252] with permission from John Wiley and Sons. d reprinted with permission from. Ref. [305].Copyright 2010 American Chemical Society.

Being similar with the hybrid organic/inorganic solar cells, the PEC solar cells is a facile method for achieving cost-effective solar cells by immersing the substrates into a liquid electrolyte. The primarily challenge for this cost-effective PEC solar cell is the surface photooxidation and photocorrosion of the silicon surface when immersed in the electrolyte. Impurities and defects are inevitably introduced in chemically grown SiNWs that can generate surface trap states and unavoidable impair the performance of devices. Also, the presence of shunt paths deteriorate the fill factor. Meanwhile, the majority of reported devices are unstable. In order to eliminate surface trap states in nanowire photoelectrodes, surface passivation can be utilized [310]. However, it may malfunction because electrical conductivity is critical for PEC solar cells. a-Si and alkyl group are good choices. Currently, the low efficiency and stability are the main consideration for designing PEC solar cells.

Beyond the SiNW solar cells we discussed above, we have provided a record efficiency based on SiNW solar cells in Table 3, including fabrication method and test condition employed in recent literature.

Table 3 A summary of high efficiency SiNW based solar cells. The ultimate efficiencies are highlighted and the method, test condition and category are given.

\begin{tabular}{|c|c|c|c|c|}
\hline Ref. & Efficiency (\%) & Method & Test condition & Category \\
\hline [249] & 17.11 & MAC & $\begin{array}{l}\text { AM 1.5G; } 100 \\
\mathrm{~mW} \mathrm{cm-2}\end{array}$ & $\begin{array}{l}\text { Radial junction } \\
\text { SiNW solar cell }\end{array}$ \\
\hline [286] & 13.2 & MAC & $\begin{array}{l}\text { AM 1.5G; } 100 \\
m W \text { cm-2 }\end{array}$ & $\begin{array}{l}\text { Organic/Inorganic } \\
\text { SiNW solar cell }\end{array}$ \\
\hline [297] & 12.9 & MAC & $\begin{array}{l}\text { AM 1.5G; } 100 \\
\mathrm{~mW} \mathrm{cm-2}\end{array}$ & HIT SiNW solar cell \\
\hline [287] & 13.36 & MAC & $\begin{array}{l}\text { AM 1.5G; } 100 \\
\text { mW cm-2 }\end{array}$ & $\begin{array}{l}\text { Organic/Inorganic } \\
\text { nanotip solar cell }\end{array}$ \\
\hline [34] & 3.4 & VLS & $\begin{array}{l}\text { AM 1.5G; } 100 \\
\mathrm{~mW} \mathrm{cm-2}\end{array}$ & $\begin{array}{l}\text { Single SiNW solar } \\
\text { cell }\end{array}$ \\
\hline [252] & 10.86 & MAC & $\begin{array}{l}\text { AM 1.5G; } 100 \\
\mathrm{~mW} \mathrm{cm-2}\end{array}$ & $\begin{array}{l}\text { SiNW } \\
\text { Photoelectrochemical } \\
\text { solar Cells }\end{array}$ \\
\hline
\end{tabular}

\section{Concluding Remarks and Outlook}

SiNW arrays promise to reduce manufacturing costs and increase the power conversion efficiency 
of photovoltaic devices. So far, however, photovoltaic cells based on SiNWs exhibit lower efficiencies than their bulk counterparts. The major problems plaguing SiNWs solar cell are due to unoptimized design and poor material quality. To date, considerable progress has been made in improving the performance of solar cells based on SiNW arrays. Both theoretical analysis and experimental realization of devices made from SiNW arrays have been widely investigated. Most of the work, if not all, indicates that the optical design and material quality play a key role in the the success of nanowire solar cells. In this review, the optical properties of SiNW arrays are first summarized to guide the geometrical design for high efficiency nanowire solar cells. Following that, representative growth methods, including both bottom-up and top-down techniques, such as VLS growth mechanism and MAC etching, are discussed. And finally, diversified studies are reviewed to demonstrate recent progress of achieving inexpensive SiNW solar cells. In conclusion, there are five parameters to achieve cost-effective solar cells when design SiNW solar cell. (i) nanowire arrangement (period, order or disorder, tilted or vertically stranding), (ii) shape, size and length, (iii) radial structure, (iv) surface treatment, (v) top contact configuration.

To sum up, there are three challenges facing to achieve cost-effective SiNW solar cells: (i) how to prepare cost-effective NWs with high quality using industrial-grade fabrication; (ii) how to collect carriers without losses that mainly caused by surface recombination; (iii) how to realize high quality $p$ - $n$ junctions. Two essential considerations point directions towards efficient solar cells: (i) less material and energy consumption. The radial $p$ - $n$ junction architecture is a good candidate for inexpensive solar cells due to low quality material use and satisfying optical absorption and carrier collection. Another possible solution is to transfer the aligned NWs to cheaper substrates such as plastic sheets, conductive glasses, metal foils. Moreover, hybrid SiNW solar cells can be fabricated at a lower temperature and thus lessen energy consumption; (ii) higher optical and electrical performance. Many routes can achieve this goal, such as incorporating with plasmonic, III-V-NWs/Si hybrid device and surface passivation, etc. Our research aims should be focus at following problems for abovementioned solar cells: (i) inexpensive contact technology and uncomplicated interconnection for single nanowires; (ii) obtaining scattering objects with high effective cross section over the available solar spectrum and facile coupling method for incorporating plasmonic source on the dense nanowires for plasmonic SiNW solar cells; (iii) stability for hybrid SiNW solar cells; (iv) stability and nontoxic\& incorrosive liquid electrolytes for PEC SiNW solar cell.

Although SiNW solar cells are not ripe for large-scale application under current technology compared with their planar Si counterparts, the SiNW solar cells will facilitate the transformation of energy structure and carbon dioxide mitigation in the near future.

\section{Acknowledgements}

The authors acknowledge the financial support from the National Natural Science Foundation of China through Grant NSFC- 61474015, NSFC-51272038, and NSFC61204060 and the National Basic Research Program (973) of China (2013CB933301), and Fundamental Research Funds for the Central Universities. 


\section{References}

[1] BP, Statistical Review of World Energy, (2013).

[2] R.M. Swanson, Photovoltaic Specialists Conference, 2005. Conference Record of the Thirty-first IEEE2005, pp. 889-894.

[3] S.A. Mann, M.J. de Wild-Scholten, V.M. Fthenakis, W.G.J.H.M. van Sark, W.C. Sinke, Progress in Photovoltaics: Research and Applications, 22 (2014) 1180-1194.

[4] B.N.E.F.p. energytrend.com, Annual Average Imported Crude Oil Price, (2015).

[5] EIA, EIA Short-Term Energy Outlook, Nov. 2015, (2015).

[6] J. Nelson, A. Gambhir, N.E.-D. ukes, Grantham Institute for Climate Change Briefing paper No 11, (2014).

[7] BP, 2014 World Energy Outlook, (2014).

[8] Silicon, USGS Mineral Commodities Survey, (2008).

[9] M.G. Mauk, J.A. Rand, R. Jonczyk, R.B. Hall, A.M. Barnett, Proceedings of the 3rd World Conference on Photovoltaic Energy Conversion2003, pp. 939-942.

[10] E.A. Alsema, Progress in Photovoltaics: Research and Applications, 8 (2000) 17-25.

[11] C.-M. Hsu, C. Battaglia, C. Pahud, Z. Ruan, F.-J. Haug, S. Fan, C. Ballif, Y. Cui, Advanced Energy Materials, 2 (2012) 628-633.

[12] K.H. Tsui, Q. Lin, H. Chou, Q. Zhang, H. Fu, P. Qi, Z. Fan, Advanced materials, 26 (2014) 28052811.

[13] I. Repins, M.A. Contreras, B. Egaas, C. DeHart, J. Scharf, C.L. Perkins, B. To, R. Noufi, Prog. Photovoltaics, 16 (2008) 235-239.

[14] H. Hoppe, N.S. Sariciftci, Journal of Materials Research, 19 (2004) 1924-1945.

[15] M.C. Beard, K.P. Knutsen, P.R. Yu, J.M. Luther, Q. Song, W.K. Metzger, R.J. Ellingson, A.J. Nozik, Nano Letters, 7 (2007) 2506-2512.

[16] R.J. Ellingson, M.C. Beard, J.C. Johnson, P. Yu, O.I. Micic, A.J. Nozik, A. Shabaev, A.L. Efros, Nano Letters, 5 (2005) 865-871.

[17] O.E. Semonin, J.M. Luther, S. Choi, H.Y. Chen, J.B. Gao, A.J. Nozik, M.C. Beard, Science, 334 (2011) 1530-1533.

[18] A. Luque, A. Marti, N. Lopez, E. Antolin, E. Canovas, C. Stanley, C. Farmer, L.J. Caballero, L. Cuadra, J.L. Balenzategui, Applied Physics Letters, 87 (2005) 083505.

[19] G.D. Wei, S.R. Forrest, Nano Letters, 7 (2007) 218-222.

[20] J. Wu, D.L. Shao, Z.H. Li, M.O. Manasreh, V.P. Kunets, Z.M. Wang, G.J. Salamo, Applied Physics Letters, 95 (2009) 3.

[21] Y.Y. Cheng, B. Fuckel, R.W. MacQueen, T. Khoury, R. Clady, T.F. Schulze, N.J. Ekins-Daukes, M.J. Crossley, B. Stannowski, K. Lips, T.W. Schmidt, Energy \& Environmental Science, 5 (2012) 6953-6959.

[22] F. Wang, R. Deng, J. Wang, Q. Wang, Y. Han, H. Zhu, X. Chen, X. Liu, Nature Materials, 10 (2011) 968-973.

[23] F. Zhang, R.C. Che, X.M. Li, C. Yao, J.P. Yang, D.K. Shen, P. Hu, W. Li, D.Y. Zhao, Nano Letters, 12 (2012) 2852-2858.

[24] W. Zou, C. Visser, J.A. Maduro, M.S. Pshenichnikov, J.C. Hummelen, Nature Photonics, 6 (2012) 
560-564.

[25] C.Y. Huang, D.Y. Wang, C.H. Wang, Y.T. Chen, Y.T. Wang, Y.T. Jiang, Y.J. Yang, C.C. Chen, Y.F. Chen, Acs Nano, 4 (2010) 5849-5854.

[26] B.S. Richards, Solar Energy Materials and Solar Cells, 90 (2006) 1189-1207.

[27] N.J. Ekins-Daukes, J.M. Barnes, K.W.J. Barnham, J.P. Connolly, M. Mazzer, J.C. Clark, R. Grey, G. Hill, M.A. Pate, J.S. Roberts, Solar Energy Materials and Solar Cells, 68 (2001) 71-87.

[28] N.J. Ekins-Daukes, K.W.J. Barnham, J.P. Connolly, J.S. Roberts, J.C. Clark, G. Hill, M. Mazzer, Applied Physics Letters, 75 (1999) 4195-4197.

[29] Z.H. Chen, Y.B. Tang, C.P. Liu, Y.H. Leung, G.D. Yuan, L.M. Chen, Y.Q. Wang, I. Bello, J.A. Zapien, W.J. Zhang, C.S. Lee, S.T. Lee, Journal of Physical Chemistry C, 113 (2009) 13433-13437.

[30] L.E. Greene, M. Law, B.D. Yuhas, P. Yang, Journal of Physical Chemistry C, 111 (2007) 1845118456.

[31] W.U. Huynh, J.J. Dittmer, A.P. Alivisatos, Science, 295 (2002) 2425-2427.

[32] J. Jiu, F. Wang, S. Isoda, M. Adachi, Chemistry Letters, 34 (2005) 1506-1507.

[33] K. Takanezawa, K. Tajima, K. Hashimoto, Applied Physics Letters, 93 (2008) 063308.

[34] B. Tian, X. Zheng, T.J. Kempa, Y. Fang, N. Yu, G. Yu, J. Huang, C.M. Lieber, Nature, 449 (2007) 885-889.

[35] F. Voigt, T. Stelzner, S. Christiansen, Progress in Photovoltaics: Research and Applications, 21 (2013) 1567-1579.

[36] J. Arbiol, B. Kalache, P.R.I. Cabarrocas, J.R. Morante, A.F.I. Morral, Nanotechnology, 18 (2007) 305606.

[37] B.P. Azeredo, J. Sadhu, J. Ma, K. Jacobs, J. Kim, K. Lee, J.H. Eraker, X. Li, S. Sinha, N. Fang, P. Ferreira, K. Hsu, Nanotechnology, 24 (2013).

[38] H. Bao, X. Ruan, Optics Letters, 35 (2010) 3378-3380.

[39] J.B. Baxter, E.S. Aydil, Applied Physics Letters, 86 (2005) 1-3.

[40] T.E. Bogart, S. Dey, K.K. Lew, S.E. Mohney, J.M. Redwing, Advanced materials, 17 (2005) 114117.

[41] L. Cao, P. Fan, A.P. Vasudev, J.S. White, Z. Yu, W. Cai, J.A. Schuller, S. Fan, M.L. Brongersma, Nano Letters, 10 (2010) 439-445.

[42] H. Chen, H. Wang, X.-H. Zhang, C.-S. Lee, S.-T. Lee, Nano Letters, 10 (2010) 864-868.

[43] Y.K. Choi, J. Zhu, J. Grunes, J. Bokor, G.A. Somorjai, Journal of Physical Chemistry B, 107 (2003) 3340-3343.

[44] G.E. Cirlin, A.D. Bouravleuv, I.P. Soshnikov, Y.B. Samsonenko, V.G. Dubrovskii, E.M. Arakcheeva, E.M. Tanklevskaya, P. Werner, Nanoscale Research Letters, 5 (2010) 360-363.

[45] Y. Cui, L.J. Lauhon, M.S. Gudiksen, J. Wang, C.M. Lieber, Applied Physics Letters, 78 (2001) 2214-2216.

[46] Y. Cui, Q. Wei, H. Park, C.M. Lieber, Science, 293 (2001) 1289-1292.

[47] J.A. Czaban, D.A. Thompson, R.R. LaPierre, Nano Letters, 9 (2009) 148-154.

[48] Q.G. Du, C.H. Kam, H.V. Demir, H.Y. Yu, X.W. Sun, Optics Letters, 36 (2011) 1884-1886.

[49] X. Duan, Y. Huang, R. Agarwal, C.M. Lieber, Nature, 421 (2003) 241-245.

[50] B. Fuhrmann, H.S. Leipner, H.R. Höche, L. Schubert, P. Werner, U. Gösele, Nano Letters, 5 (2005) 2524-2527.

[51] D. Gao, R.R. He, C. Carraro, R.T. Howe, P.D. Yang, R. Maboudian, Journal of the American Chemical Society, 127 (2005) 4574-4575. 
[52] J. Goldberger, A.I. Hochbaum, R. Fan, P. Yang, Nano Letters, 6 (2006) 973-977.

[53] X. Gong, Y. Jiang, M. Li, H. Liu, H. Ma, RSC Adv., 5 (2015) 10310-10317.

[54] T. Gorisse, L. Dupre, P. Gentile, M. Martin, M. Zelsmann, D. Buttard, Nanoscale Research Letters, 8(1) (2013) 287.

[55] M.S. Gudiksen, L.J. Lauhon, J. Wang, D.C. Smith, C.M. Lieber, Nature, 415 (2002) 617-620.

[56] O. Gunawan, S. Guha, Solar Energy Materials and Solar Cells, 93 (2009) 1388-1393.

[57] L. He, D. Lai, H. Wang, C. Jiang, Rusli, Small, 8 (2012) 1664-1668.

[58] A.T. Heitsch, D.D. Fanfair, H.Y. Tuan, B.A. Korgel, Journal of the American Chemical Society, 130 (2008) 5436-5437.

[59] A.I. Hochbaum, R. Fan, R. He, P. Yang, Nano Letters, 5 (2005) 457-460.

[60] N.M. Gabor, Z. Zhong, K. Bosnick, J. Park, P.L. McEuen, Science, 325 (2009) 1367-1371.

[61] Y. Jia, J. Wei, K. Wang, A. Cao, Q. Shu, X. Gui, Y. Zhu, D. Zhuang, G. Zhang, B. Ma, L. Wang, W. Liu, Z. Wang, J. Luo, D. Wu, Advanced materials, 20 (2008) 4594-4598.

[62] G.K. Mor, K. Shankar, M. Paulose, O.K. Varghese, C.A. Grimes, Nano Letters, 6 (2006) 215-218.

[63] J. Wei, Y. Jia, Q. Shu, Z. Gu, K. Wang, D. Zhuang, G. Zhang, Z. Wang, J. Luo, A. Cao, D. Wu, Nano Letters, 7 (2007) 2317-2321.

[64] K. Zhu, N.R. Neale, A. Miedaner, A.J. Frank, Nano Letters, 7 (2007) 69-74.

[65] J.U. Lee, Applied Physics Letters, 87 (2005) 073101.

[66] S. Buhbut, S. Itzhakov, E. Tauber, M. Shalom, I. Hod, T. Geiger, Y. Garini, D. Oron, A. Zaban, Acs Nano, 4 (2010) 1293-1298.

[67] X.J. Hao, E.C. Cho, G. Scarder, Y.S. Shen, E. Bellet-Amalric, D. Bellet, G. Conibeer, M.A. Green, Solar Energy Materials and Solar Cells, 93 (2009) 1524-1530.

[68] R.B. Laghumavarapu, M. El-Emawy, N. Nuntawong, A. Moscho, L.F. Lester, D.L. Huffaker, Applied Physics Letters, 91 (2007) 3.

[69] Y.L. Lee, Y.S. Lo, Advanced Functional Materials, 19 (2009) 604-609.

[70] J. Wu, P. Yu, A.S. Susha, K.A. Sablon, H. Chen, Z. Zhou, H. Li, H. Ji, X. Niu, A.O. Govorov, A.L. Rogach, Z.M. Wang, Nano Energy, 13 (2015) 827-835.

[71] P.V. Kamat, Journal of Physical Chemistry C, 112 (2008) 18737-18753.

[72] V.R. Reddy, J. Wu, M.O. Manasreh, Materials Letters, 92 (2013) 296-299.

[73] H.Y. Tuan, D.C. Lee, T. Hanrath, B.A. Korgel, Nano Letters, 5 (2005) 681-684.

[74] Y. Cui, C.M. Lieber, Science, 291 (2001) 851-853.

[75] C.K. Chan, H. Peng, G. Liu, K. Mcllwrath, X.F. Zhang, R.A. Huggins, Y. Cui, Nature Nanotechnology, 3 (2008) 31-35.

[76] G. Zheng, F. Patolsky, Y. Cui, W.U. Wang, C.M. Lieber, Nature Biotechnology, 23 (2005) 12941301.

[77] A.I. Hochbaum, R. Chen, R.D. Delgado, W. Liang, E.C. Garnett, M. Najarian, A. Majumdar, P. Yang, Nature, 451 (2008) 163-167.

[78] B.M. Kayes, H.A. Atwater, N.S. Lewis, Journal of Applied Physics, 97 (2005) 114302.

[79] K.-Q. Peng, X. Wang, L. Li, Y. Hu, S.-T. Lee, Nano Today, 8 (2013) 75-97.

[80] L. Hu, G. Chen, Nano Letters, 7 (2007) 3249-3252.

[81] J. Li, H. Yu, S.M. Wong, X. Li, G. Zhang, P.G.Q. Lo, D.L. Kwong, Applied Physics Letters, 95 (2009) 243113.

[82] J. Li, H. Yu, S.M. Wong, G. Zhang, X. Sun, P.G.Q. Lo, D.L. Kwong, Applied Physics Letters, 95 (2009) 033102. 
[83] J. Li, H. Yu, Y. Li, Nanotechnology, 23 (2012) 194010.

[84] B.C.P. Sturmberg, K.B. Dossou, L.C. Botten, A.A. Asatryan, C.G. Poulton, C.M. De Sterke, R.C. McPhedran, Opt. Express, 19 (2011) A1067-A1081.

[85] T.J. Kempa, J.F. Cahoon, S.-K. Kim, R.W. Day, D.C. Bell, H.-G. Park, C.M. Lieber, Proceedings of the National Academy of Sciences of the United States of America, 109 (2012) 1407-1412.

[86] Y. Li, Q. Chen, D. He, J. Li, Nano Energy, 7 (2014) 10-24.

[87] C. Lin, M.L. Povinelli, Opt. Express, 17 (2009) 19371-19381.

[88] K.T. Fountaine, W.S. Whitney, H.A. Atwater, Journal of Applied Physics, 116 (2014) 153106.

[89] S.-K. Kim, R.W. Day, J.F. Cahoon, T.J. Kempa, K.-D. Song, H.-G. Park, C.M. Lieber, Nano Letters, 12 (2012) 4971-4976.

[90] S.-K. Kim, K.-D. Song, T.J. Kempa, R.W. Day, C.M. Lieber, H.-G. Park, Acs Nano, 8 (2014) 3707-3714.

[91] S. Sandhu, Z. Yu, S. Fan, Nano Lett, 14 (2014) 1011-1015.

[92] Y.-F. Huang, S. Chattopadhyay, Y.-J. Jen, C.-Y. Peng, T.-A. Liu, Y.-K. Hsu, C.-L. Pan, H.-C. Lo, C.-H. Hsu, Y.-H. Chang, C.-S. Lee, K.-H. Chen, L.-C. Chen, Nature Nanotechnology, 2 (2007) 770774.

[93] S. Jeong, E.C. Garnett, S. Wang, Z. Yu, S. Fan, M.L. Brongersma, M.D. McGehee, Y. Cui, Nano Lett, 12 (2012) 2971-2976.

[94] S.E. Han, G. Chen, Nano Letters, 10 (2010) 4692-4696.

[95] C.H. Hsu, H.C. Lo, C.F. Chen, C.T. Wu, J.S. Hwang, D. Das, J. Tsai, L.C. Chen, K.H. Chen, Nano Letters, 4 (2004) 471-475.

[96] J. Zhu, Z. Yu, G.F. Burkhard, C.-M. Hsu, S.T. Connor, Y. Xu, Q. Wang, M. McGehee, S. Fan, Y. Cui, Nano Letters, 9 (2009) 279-282.

[97] Z. Fan, R. Kapadia, P.W. Leu, X. Zhang, Y.-L. Chueh, K. Takei, K. Yu, A. Jamshidi, A.A. Rathore, D.J. Ruebusch, M. Wu, A. Javey, Nano Letters, 10 (2010) 3823-3827.

[98] B. Hua, B. Wang, M. Yu, P.W. Leu, Z. Fan, Nano Energy, 2 (2013) 951-957.

[99] R.A. Street, P. Qi, R. Lujan, W.S. Wong, Applied Physics Letters, 93 (2008) 163109.

[100] R.A. Street, W.S. Wong, C. Paulson, Nano Letters, 9 (2009) 3494-3497.

[101] B.C.P. Sturmberg, K.B. Dossou, L.C. Botten, A.A. Asatryan, C.G. Poulton, R.C. McPhedran, C. Martijn De Sterke, Applied Physics Letters, 101 (2012) 173902.

[102] C. Lin, M.L. Povinelli, Opt. Express, 19 (2011) A1148-A1154.

[103] W. Wang, Q. Zhao, K. Laurent, Y. Leprince-Wang, Z.M. Liao, D. Yu, Nanoscale, 4 (2012) 261268.

[104] S.L. Diedenhofen, G. Vecchi, R.E. Algra, A. Hartsuiker, O.L. Muskens, G. Immink, E.P.A.M. Bakkers, W.L. Vos, J.G. Rivas, Advanced materials, 21 (2009) 973-978.

[105] H. Bao, X. Ruan, 2010, pp. 77721J-77721J-77727.

[106] L. Hong, Rusli, X. Wang, H. Zheng, H. Wang, H. Yu, Journal of Applied Physics, 114 (2013) 084303.

[107] M.I. Kayes, P.W. Leu, Nanoscale Research Letters, 9 (2014) 1-6.

[108] Y. Wu, X. Yan, X. Zhang, X. Ren, Opt. Express, 23 (2015) A1603-A1612.

[109] O. Kilic, M. Digonnet, G. Kino, O. Solgaard, Opt. Express, 16 (2008) 13090-13103.

[110] K. Peng, M. Zhang, A. Lu, N.-B. Wong, R. Zhang, S.-T. Lee, Applied Physics Letters, 90 (2007) 163123.

[111] Z. Huang, T. Shimizu, S. Senz, Z. Zhang, N. Geyer, U. Goesele, Journal of Physical Chemistry C, 
114 (2010) 10683-10690.

[112] H. Fang, X. Li, S. Song, Y. Xu, J. Zhu, Nanotechnology, 19 (2008).

[113] L. Cheng, W. Dou, S. Bai, W. Wu, Q. Xu, Y. Qin, Science of Advanced Materials, 5 (2013) 11791183.

[114] Y. Li, M. Li, R. Li, P. Fu, B. Jiang, D. Song, C. Shen, Y. Zhao, R. Huang, Optics Communications, 355 (2015) 6-9.

[115] P. Van Trinh, D. Mrinal, B. Hung Thang, F. Naoki, Advances in Natural Sciences: Nanoscience and Nanotechnology, 5 (2014) 045014.

[116] T. Xu, Y. Lambert, C. Krzeminski, B. Grandidier, D. Stievenard, G. Leveque, A. Akjouj, Y. Pennec, B. Djafari-Rouhani, Journal of Applied Physics, 112 (2012) 7.

[117] J.D. Holmes, K.P. Johnston, R.C. Doty, B.A. Korgel, Science, 287 (2000) 1471-1473.

[118] Y. Wu, Y. Cui, L. Huynh, C.J. Barrelet, D.C. Bell, C.M. Lieber, Nano Letters, 4 (2004) 433-436.

[119] C.P. Li, C.S. Lee, X.L. Ma, N. Wang, R.Q. Zhang, S.T. Lee, Advanced materials, 15 (2003) 607609.

[120] R.S. Wagner, W.C. Ellis, Applied Physics Letters, 4 (1964) 89-90.

[121] B.M. Kayes, M.A. Filler, M.C. Putnam, M.D. Kelzenberg, N.S. Lewis, H.A. Atwater, Applied Physics Letters, 91 (2007) 103110.

[122] V.T. Renard, M. Jublot, P. Gergaud, P. Cherns, D. Rouchon, A. Chabli, V. Jousseaume, Nature Nanotechnology, 4 (2009) 654-657.

[123] B.J. Kim, J. Tersoff, S. Kodambaka, M.C. Reuter, E.A. Stach, F.M. Ross, Science, 322 (2008) 1070-1073.

[124] B.J. Kim, J. Tersoff, C.Y. Wen, M.C. Reuter, E.A. Stach, F.M. Ross, Physical Review Letters, 103 (2009) 155701.

[125] C.Y. Wen, M.C. Reuter, J. Bruley, J. Tersoff, S. Kodambaka, E.A. Stach, F.M. Ross, Science, 326 (2009) $1247-1250$.

[126] J.B. Hannon, S. Kodambaka, F.M. Ross, R.M. Tromp, Nature, 440 (2006) 69-71.

[127] S. Kodambaka, J.B. Hannon, R.M. Tromp, F.M. Ross, Nano Letters, 6 (2006) 1292-1296.

[128] J.E. Allen, E.R. Hemesath, D.E. Perea, J.L. Lensch-Falk, Z.Y. Li, F. Yin, M.H. Gass, P. Wang, A.L. Bleloch, R.E. Palmer, L.J. Lauhon, Nature Nanotechnology, 3 (2008) 168-173.

[129] O. Moutanabbir, S. Senz, R. Scholz, M. Alexe, Y. Kim, E. Pippel, Y. Wang, C. Wiethoff, T. Nabbefeld, F. Meyer Zu Heringdorf, M. Horn-Von Hoegen, ACS Nano, 5 (2011) 1313-1320.

[130] M.C. Putnam, D.B. Turner-Evans, M.D. Kelzenberg, S.W. Boettcher, N.S. Lewis, H.A. Atwater, Applied Physics Letters, 95 (2009) 163116.

[131] S. Hofmann, C. Ducati, R.J. Neill, S. Piscanec, A.C. Ferrari, J. Geng, R.E. Dunin-Borkowski, J. Robertson, Journal of Applied Physics, 94 (2003) 6005-6012.

[132] T. Kuykendall, P.J. Pauzauskie, Y.F. Zhang, J. Goldberger, D. Sirbuly, J. Denlinger, P.D. Yang, Nature Materials, 3 (2004) 524-528.

[133] L. Schubert, P. Werner, N.D. Zakharov, G. Gerth, F.M. Kolb, L. Long, U. Gösele, T.Y. Tan, Applied Physics Letters, 84 (2004) 4968-4970.

[134] N. Zakharov, P. Werner, L. Sokolov, U. Gösele, Physica E: Low-Dimensional Systems and Nanostructures, 37 (2007) 148-152.

[135] P. Werner, N.D. Zakharov, G. Gerth, L. Schubert, U. Göele, International Journal of Materials Research, 97 (2006) 1008-1015.

[136] A.M. Morales, C.M. Lieber, Science, 279 (1998) 208-211. 
[137] Y.F. Zhang, Y.H. Tang, N. Wang, D.P. Yu, C.S. Lee, I. Bello, S.T. Lee, Applied Physics Letters, 72 (1998) 1835-1837.

[138] H.Z. Zhang, D.P. Yu, Y. Ding, Z.G. Bai, Q.L. Hang, S.Q. Feng, Applied Physics Letters, 73 (1998) 3396-3398.

[139] H.Y. Peng, Z.W. Pan, L. Xu, X.H. Fan, N. Wang, S.T. Lee, Lee, Advanced materials, 13 (2001) 317-320.

[140] G.W. Zhou, Z. Zhang, Z.G. Bai, S.Q. Feng, D.P. Yu, Applied Physics Letters, 73 (1998) 677-679.

[141] N. Wang, Y.H. Tang, Y.F. Zhang, D.P. Yu, C.S. Lee, I. Bello, S.T. Lee, Chemical Physics Letters, 283 (1998) 368-372.

[142] R.Q. Zhang, Y. Lifshitz, S.T. Lee, Advanced materials, 15 (2003) 635-640.

[143] Y. Wang, V. Schmidt, S. Senz, U. Gösele, Nature nanotechnology, 1 (2006) 186-189.

[144] C.Y. Wen, M.C. Reuter, J. Tersoff, E.A. Stach, F.M. Ross, Nano Letters, 10 (2010) 514-519.

[145] Y.Y. Zhang, M. Aagesen, J.V. Holm, H.I. Jorgensen, J. Wu, H.Y. Liu, Nano Letters, 13 (2013) 3897-3902.

[146] R. Laocharoensuk, K. Palaniappan, N.A. Smith, R.M. Dickerson, D.J. Werder, J.K. Baldwin, J.A. Hollingsworth, Nature Nanotechnology, 8 (2013) 660-666.

[147] H. Yu, W.E. Buhro, Advanced materials, 15 (2003) 416-419.

[148] T.J. Trentler, K.M. Hickman, S.C. Goel, A.M. Viano, P.C. Gibbons, W.E. Buhro, Science, 270 (1995) 1791-1794.

[149] T. Hanrath, B.A. Korgel, Advanced materials, 15 (2003) 437-440.

[150] S. Th, G. Andrä, E. Wendler, W. Wesch, R. Scholz, U. Gösele, S. Christiansen, Nanotechnology, 17 (2006) 2895.

[151] N. Wang, Y.F. Zhang, Y.H. Tang, C.S. Lee, S.T. Lee, Applied Physics Letters, 73 (1998) 39023904.

[152] W.S. Shi, H.Y. Peng, Y.F. Zheng, N. Wang, N.G. Shang, Z.W. Pan, C.S. Lee, S.T. Lee, Advanced materials, 12 (2000) 1343-1345.

[153] S.T. Lee, N. Wang, Y.F. Zhang, Y.H. Tang, MRS Bulletin, 24 (1999) 36-42.

[154] B.A. Wacaser, M.C. Reuter, M.M. Khayyat, C.Y. Wen, R. Haight, S. Guha, F.M. Ross, Nano Letters, 9 (2009) 3296-3301.

[155] Y.W. Wang, V. Schmidt, S. Senz, U. Gosele, Nature Nanotechnology, 1 (2006) 186-189.

[156] Y. Yao, S. Fan, Materials Letters, 61 (2007) 177-181.

[157] E.C. Garnett, W. Liang, P. Yang, Advanced materials, 19 (2007) 2946-2950.

[158] H. Pan, S. Lim, C. Poh, H. Sun, X. Wu, Y. Feng, J. Lin, Nanotechnology, 16 (2005) 417.

[159] S.N. Mohammad, J. Chem. Phys., 131 (2009) 13.

[160] E.I. Givargizov, Journal of Crystal Growth, 31 (1975) 20-30.

[161] M.E. Messing, K. Hillerich, J. Bolinsson, K. Storm, J. Johansson, K.A. Dick, K. Deppert, Nano Research, 3 (2010) 506-519.

[162] T. Mårtensson, P. Carlberg, M. Borgström, L. Montelius, W. Seifert, L. Samuelson, Nano Letters, 4 (2004) 699-702.

[163] C.W. Pinion, D.P. Nenon, J.D. Christesen, J.F. Cahoon, Acs Nano, 8 (2014) 6081-6088.

[164] S. Kodambaka, J. Tersoff, M.C. Reuter, F.M. Ross, Physical Review Letters, 96 (2006) 096105.

[165] V. Schmidt, S. Senz, U. Goesele, Physical Review B, 75 (2007) 045335.

[166] V.G. Dubrovskii, N.V. Sibirev, G.E. Cirlin, I.P. Soshnikov, W.H. Chen, R. Larde, E. Cadel, P. Pareige, T. Xu, B. Grandidier, J.P. Nys, D. Stievenard, M. Moewe, L.C. Chuang, C. Chang-Hasnain, 
Physical Review B, 79 (2009) 205316.

[167] X.Y. Zhang, L.D. Zhang, G.W. Meng, G.H. Li, N.Y. Jin-Phillipp, F. Phillipp, Advanced materials, 13 (2001) 1238-1241.

[168] T. Shimizu, T. Xie, J. Nishikawa, S. Shingubara, S. Senz, U. Goesele, Advanced materials, 19 (2007) 917-920.

[169] I. Lombardi, A.I. Hochbaum, P.D. Yang, C. Carraro, R. Maboudian, Chemistry of Materials, 18 (2006) 988-991.

[170] M. Hocevar, G. Immink, M. Verheijen, N. Akopian, V. Zwiller, L. Kouwenhoven, E. Bakkers, Nat Commun, 3 (2012) 1266.

[171] R. Juhasz, N. Elfström, J. Linnros, Nano Letters, 5 (2005) 275-280.

[172] H.D. Tong, S. Chen, W.G. Van Der Wiel, E.T. Carlen, A.D. Van Berg, Nano Letters, 9 (2009) 1015-1022.

[173] K.J. Morton, G. Nieberg, S. Bai, S.Y. Chou, Nanotechnology, 19 (2008) 345301.

[174] K.Q. Peng, Y.J. Yan, S.P. Gao, J. Zhu, Advanced materials, 14 (2002) 1164-1167.

[175] Z. Huang, H. Fang, J. Zhu, Advanced materials, 19 (2007) 744-748.

[176] W.K. Choi, T.H. Liew, M.K. Dawood, H.I. Smith, C.V. Thompson, M.H. Hong, Nano Letters, 8 (2008) 3799-3802.

[177] Y. Hu, K.-Q. Peng, Z. Qiao, X. Huang, F.-Q. Zhang, R.-N. Sun, X.-M. Meng, S.-T. Lee, Nano Letters, 14 (2014) 4212-4219.

[178] O.J. Hildreth, D.R. Schmidt, Advanced Functional Materials, 24 (2014) 3827-3833.

[179] Y. Hu, K.-Q. Peng, L. Liu, Z. Qiao, X. Huang, X.-L. Wu, X.-M. Meng, S.-T. Lee, Scientific Reports, 4 (2014) 3667.

[180] D. DimovaMalinovska, M. SendovaVassileva, N. Tzenov, M. Kamenova, Thin Solid Films, 297 (1997) 9-12.

[181] H. Morinaga, M. Suyama, T. Ohmi, Journal of the Electrochemical Society, 141 (1994) 28342841.

[182] X. Li, P.W. Bohn, Applied Physics Letters, 77 (2000) 2572-2574.

[183] K.S. Sanjay, K. Dinesh, S.W. Schmitt, K.N. Sood, S.H. Christiansen, P.K. Singh, Nanotechnology, 25 (2014) 175601.

[184] K. Peng, A. Lu, R. Zhang, S.T. Lee, Advanced Functional Materials, 18 (2008) 3026-3035.

[185] Z. Huang, N. Geyer, P. Werner, J. de Boor, U. Goesele, Advanced materials, 23 (2011) 285-308.

[186] Y. Qu, L. Liao, Y. Li, H. Zhang, Y. Huang, X. Duan, Nano Letters, 9 (2009) 4539-4543.

[187] S. Shu-Chia, S. Hong-Jhang, H. Shih-Che, L. Ching-Fuh, Nanotechnology (IEEE-NANO), 2010 10th IEEE Conference on2010, pp. 474-477.

[188] J.M. Weisse, C.H. Lee, D.R. Kim, X. Zheng, Nano Letters, 12 (2012) 3339-3343.

[189] C.H. Lee, D.R. Kim, X. Zheng, Nano Letters, 11 (2011) 3435-3439.

[190] J.M. Weisse, C.H. Lee, D.R. Kim, L. Cai, P.M. Rao, X. Zheng, Nano Letters, 13 (2013) 43624368.

[191] K.E. Plass, M.A. Filler, J.M. Spurgeon, B.M. Kayes, S. Maldonado, B.S. Brunschwig, H.A. Atwater, N.S. Lewis, Advanced materials, 21 (2009) 325-328.

[192] J.-S. Huang, C.-Y. Hsiao, S.-J. Syu, J.-J. Chao, C.-F. Lin, Solar Energy Materials and Solar Cells, 93 (2009) 621-624.

[193] V. Sivakov, G. Andra, A. Gawlik, A. Berger, J. Plentz, F. Falk, S.H. Christiansen, Nano Letters, 9 (2009) 1549-1554. 
[194] K.Q. Peng, Y. Wu, H. Fang, X.Y. Zhong, Y. Xu, J. Zhu, Angewandte Chemie-International Edition, 44 (2005) 2737-2742.

[195] K. Peng, J. Hu, Y. Yan, Y. Wu, H. Fang, Y. Xu, S. Lee, J. Zhu, Advanced Functional Materials, 16 (2006) 387-394.

[196] C.-Y. Chen, C.-S. Wu, C.-J. Chou, T.-J. Yen, Advanced materials, 20 (2008) 3811-3815.

[197] Z. Huang, T. Shimizu, S. Senz, Z. Zhang, X. Zhang, W. Lee, N. Geyer, U. Goesele, Nano Letters, 9 (2009) 2519-2525.

[198] H. Fang, Y. Wu, J. Zhao, J. Zhu, Nanotechnology, 17 (2006) 3768-3774.

[199] C.-L. Lee, K. Tsujino, Y. Kanda, S. Ikeda, M. Matsumura, Journal of Materials Chemistry, 18 (2008) 1015-1020.

[200] K. Tsujino, M. Matsumura, Advanced materials, 17 (2005) 1045-1047.

[201] G. Venturi, A. Castaldini, A. Schleusener, V. Sivakov, A. Cavallini, Nanotechnology, 26 (2015) 195705-195705.

[202] S. Li, W. Ma, Y. Zhou, X. Chen, Y. Xiao, M. Ma, W. Zhu, F. Wei, Nanoscale Research Letters, 9 (2014) 196.

[203] O.J. Hildreth, W. Lin, C.P. Wong, Acs Nano, 3 (2009) 4033-4042.

[204] M.K. Dawood, S. Tripathy, S.B. Dolmanan, T.H. Ng, H. Tan, J. Lam, Journal of Applied Physics, 112 (2012) 073509.

[205] S. Zhang, X. Wang, H. Liu, W. Shen, Nanotechnology, 25 (2014) 025602.

[206] Y. Kim, A. Tsao, D.H. Lee, R. Maboudian, Journal of Materials Chemistry C, 1 (2013) 220-224.

[207] B. Mikhael, B. Elise, M. Xavier, S. Sebastian, M. Johann, P. Laetitia, ACS applied materials \& interfaces, 3 (2011) 3866-3873.

[208] J. Yeom, D. Ratchford, C.R. Field, T.H. Brintlinger, P.E. Pehrsson, Advanced Functional Materials, 24 (2014) 106-116.

[209] Z. Wu, Z. Wang, S. Wang, Z. Zhong, Nanoscale Research Letters, 9 (2014) 495.

[210] J.-Y. Choi, T.L. Alford, C.B. Honsberg, Langmuir, 31 (2015) 4018-4023.

[211] D. Teng, L. Wu, W. He, C. Ye, Langmuir, 30 (2014) 2259-2265.

[212] H. Lin, H.-Y. Cheung, F. Xiu, F. Wang, S. Yip, N. Han, T. Hung, J. Zhou, J.C. Ho, C.-Y. Wong, Journal of Materials Chemistry A, 1 (2013) 9942-9946.

[213] H. Lin, F. Xiu, M. Fang, S. Yip, H.-Y. Cheung, F. Wang, N. Han, K.S. Chan, C.-Y. Wong, J.C. Ho, Acs Nano, 8 (2014) 3752-3760.

[214] W. Chern, K. Hsu, I.S. Chun, B.P. de Azeredo, N. Ahmed, K.-H. Kim, J.-m. Zuo, N. Fang, P. Ferreira, X. Li, Nano Letters, 10 (2010) 1582-1588.

[215] Z. Huang, X. Zhang, M. Reiche, L. Liu, W. Lee, T. Shimizu, S. Senz, U. Goesele, Nano Letters, 8 (2008) 3046-3051.

[216] J. Kim, H. Han, Y.H. Kim, S.-H. Choi, J.-C. Kim, W. Lee, Acs Nano, 5 (2011) 3222-3229.

[217] H.C. Jeon, C.-J. Heo, S.Y. Lee, S.-M. Yang, Advanced Functional Materials, 22 (2012) 42684274.

[218] S.-W. Chang, V.P. Chuang, S.T. Boles, C.A. Ross, C.V. Thompson, Advanced Functional Materials, 19 (2009) 2495-2500.

[219] E. Garnett, P. Yang, Nano Lett, 10 (2010) 1082-1087.

[220] K. Seo, M. Wober, P. Steinvurzel, E. Schonbrun, Y. Dan, T. Ellenbogen, K.B. Crozier, Nano Letters, 11 (2011) 1851-1856.

[221] P. Spinelli, M.A. Verschuuren, A. Polman, Nature Communications, 3 (2012) 692. 
[222] E.A. Costner, M.W. Lin, W.-L. Jen, C.G. Willson, Annual Review of Materials Research, 39 (2009) 155-180.

[223] Y. Lu, A. Lal, Nano Letters, 10 (2010) 4651-4656.

[224] Y. Lu, A. Lal, Nano Letters, 10 (2010) 2197-2201.

[225] C.W. Kuo, J.Y. Shiu, P.L. Chen, G.A. Somorjai, Journal of Physical Chemistry B, 107 (2003) 9950-9953.

[226] C.M. Hsu, S.T. Connor, M.X. Tang, Y. Cui, Applied Physics Letters, 93 (2008) 133109.

[227] A. Rahman, A. Ashraf, H. Xin, X. Tong, P. Sutter, M.D. Eisaman, C.T. Black, Nature Communications, 6 (2015) 5963.

[228] S.G. Cloutier, C.H. Hsu, P.A. Kossyrev, J. Xu, Advanced materials, 18 (2006) 841-844.

[229] H.P. Yoon, Y.A. Yuwen, C.E. Kendrick, G.D. Barber, N.J. Podraza, J.M. Redwing, T.E. Mallouk, C.R. Wronski, T.S. Mayer, Applied Physics Letters, 96 (2010) 213503.

[230] M.D. Kelzenberg, S.W. Boettcher, J.A. Petykiewicz, D.B. Turner-Evans, M.C. Putnam, E.L. Warren, J.M. Spurgeon, R.M. Briggs, N.S. Lewis, H.A. Atwater, Nat Mater, 9 (2010) 239-244.

[231] J.D. Christesen, X. Zhang, C.W. Pinion, T.A. Celano, C.J. Flynn, J.F. Cahoon, Nano Letters, 12 (2012) 6024-6029.

[232] D.C. Dillen, K. Kim, E.-S. Liu, E. Tutuc, Nat Nano, 9 (2014) 116-120.

[233] L.J. Lauhon, M.S. Gudiksen, D. Wang, C.M. Lieber, Nature, 420 (2002) 57-61.

[234] J. Yoo, S.A. Dayeh, W. Tang, S.T. Picraux, Applied Physics Letters, 102 (2013) 093113.

[235] J. Jie, W. Zhang, K. Peng, G. Yuan, C.S. Lee, S.-T. Lee, Advanced Functional Materials, 18 (2008) 3251-3257.

[236] M.Y. Bashouti, K. Sardashti, S.W. Schmitt, M. Pietsch, J. Ristein, H. Haick, S.H. Christiansen, Progress in Surface Science, 88 (2013) 39-60.

[237] X. Fang, Y. Li, X.Q. Wang, J.N. Ding, N.Y. Yuan, Sol. Energy, 116 (2015) 100-107.

[238] W.C. Wang, C.W. Lin, H.J. Chen, C.W. Chang, J.J. Huang, M.J. Yang, B. Tjahjono, J.J. Huang, W.C. Hsu, M.J. Chen, Acs Applied Materials \& Interfaces, 5 (2013) 9752-9759.

[239] Y.P. Dan, K. Seo, K. Takei, J.H. Meza, A. Javey, K.B. Crozier, Nano Letters, 11 (2011) 25272532.

[240] Z.W. Zuo, K. Zhu, G.L. Cui, W.X. Huang, J. Qu, Y. Shi, Y.S. Liu, G.B. Ji, Solar Energy Materials and Solar Cells, 125 (2014) 248-252.

[241] A.D. Mallorqui, E. Alarcon-Llado, I.C. Mundet, A. Kiani, B. Demaurex, S. De Wolf, A. Menzel, M. Zacharias, A.I. Morral, Nano Research, 8 (2015) 673-681.

[242] D.R. Kim, C.H. Lee, P.M. Rao, I.S. Cho, X. Zheng, Nano Lett, 11 (2011) 2704-2708.

[243] M.-A. Tsai, P.-C. Tseng, H.-C. Chen, H.-C. Kuo, P. Yu, Opt. Express, 19 (2011) A28-A34.

[244] B. Hoex, F.J.J. Peeters, M. Creatore, M.A. Blauw, W.M.M. Kessels, M.C.M. van de Sanden, Journal of Vacuum Science \& Technology A, 24 (2006) 1823-1830.

[245] J.Y. Kim, M.K. Kwon, V.J. Logeeswaran, S. Grego, M.S. Islam, In-situ chlorine passivation to suppress surface-dominant transport in silicon nanowire devices, in: M.S. Islam, N.P. Kobayashi, A.A. Talin (Eds.) Nanoepitaxy: Homo- and Heterogeneous Synthesis, Characterization, and Device Integration of Nanomaterials Ii, Spie-Int Soc Optical Engineering, Bellingham, 2010.

[246] J.Y. Kim, M.K. Kwon, V.J. Logeeswaran, S. Grego, M.S. Islam, IEEE Trans. Nanotechnol., 11 (2012) 782-787.

[247] S. Yu, F. Roemer, B. Witzigmann, Journal of Photonics for Energy, 2 (2012) 028002-028001.

[248] M. Dutta, N. Fukata, Nano Energy, 11 (2015) 219-225. 
[249] X.X. Lin, X. Hua, Z.G. Huang, W.Z. Shen, Nanotechnology, 24 (2013) 235402.

[250] L. Junshuai, W. She Mein, L. Yali, Y. Hongyu, Photovoltaic Specialists Conference (PVSC), 2010 35th IEEE2010, pp. 001469-001473.

[251] M.-F. Ng, L.Y. Sim, H. Da, H. Jin, K.H. Lim, S.-W. Yang, Theoretical Chemistry Accounts, 127 (2010) 689-695.

[252] X. Wang, K.Q. Peng, X.J. Pan, X. Chen, Y. Yang, L. Li, X.M. Meng, W.J. Zhang, S.T. Lee, Angewandte Chemie, 50 (2011) 9861-9865.

[253] M. Triplett, Y. Yang, F. Leonard, A.A. Talin, M.S. Islam, D. Yu, Nano Lett, 15 (2015) 523-529.

[254] S. Zhong, Z. Huang, X. Lin, Y. Zeng, Y. Ma, W. Shen, Advanced materials, 27 (2015) 555-561.

[255] A. Chaudhry, V. Ramamurthi, E. Fong, M.S. Islam, Nano Letters, 7 (2007) 1536-1541.

[256] H. Han, J. Kim, H.S. Shin, J.Y. Song, W. Lee, Advanced materials, 24 (2012) 2284-2288.

[257] M. Abhishek, E.B. John, K. Sergiy, V.D. Albert, A. Geetha, V.R. Mulpuri, Nanotechnology, 22 (2011) 075206.

[258] X. Zhao, C.M. Wei, L. Yang, M.Y. Chou, Physical Review Letters, 92 (2004) 236805.

[259] A.J. Lu, R.Q. Zhang, S.T. Lee, Nanotechnology, 19 (2008) 035708.

[260] X. Li, Y. Xiao, J.H. Bang, D. Lausch, S. Meyer, P.-T. Miclea, J.-Y. Jung, S.L. Schweizer, J.-H. Lee, R.B. Wehrspohn, Advanced materials, 25 (2013) 3187-3191.

[261] P. Zheng, F.E. Rougieux, C. Samundsett, X.B. Yang, Y.M. Wan, J. Degoulange, R. Einhaus, P. Rivat, D. Macdonald, Applied Physics Letters, 108 (2016) 5.

[262] T. Schutz-Kuchly, J. Veirman, S. Dubois, D.R. Heslinga, Applied Physics Letters, 96 (2010) 3.

[263] J. Safarian, G. Tranell, M. Tangstad, Processes for upgrading metallurgical grade silicon to solar grade silicon, in: G. Tranell (Ed.) Technoport 2012 - Sharing Possibilities and 2nd Renewable Energy Research Conference, Elsevier Science Bv, Amsterdam, 2012, pp. 88-97.

[264] A.J. Lu, Point defects in the silicon nanowire, in: J. Chu, C. Wang (Eds.) Eighth International Conference on Thin Film Physics and Applications, Spie-Int Soc Optical Engineering, Bellingham, 2013.

[265] Y.H. Tang, Y.F. Zheng, C.S. Lee, S.T. Lee, Chemical Physics Letters, 328 (2000) 346-349.

[266] N.S. Lewis, Science, 315 (2007) 798-801.

[267] Z. Yang, G. Cao, A. Shang, D.Y. Lei, C. Zhang, P. Gao, J. Ye, X. Li, Nanoscale Research Letters, 11 (2016) 1-7.

[268] M.D. Kelzenberg, D.B. Turner-Evans, B.M. Kayes, M.A. Filler, M.C. Putnam, N.S. Lewis, H.A. Atwater, Nano Letters, 8 (2008) 710-714.

[269] K.Q. Peng, Y. Xu, Y. Wu, Y.J. Yan, S.T. Lee, J. Zhu, Small, 1 (2005) 1062-1067.

[270] Z.M. Liao, J. Xu, J.M. Zhang, D.P. Yu, Applied Physics Letters, 93 (2008) 023111.

[271] Y.H. Zhan, J.P. Zhao, C.H. Zhou, M. Alemayehu, Y.P. Li, Y. Li, Opt. Express, 20 (2012) 1150611516.

[272] E.C. Garnett, M.L. Brongersma, Y. Cui, M.D. McGehee, Annual Review of Materials Research, 41 (2011) 269-295.

[273] P. Krogstrup, H.I. Jørgensen, M. Heiss, O. Demichel, J.V. Holm, M. Aagesen, J. Nygard, A. Fontcuberta i Morral, Nature Photonics, 7 (2013) 306-310.

[274] H.A. Atwater, A. Polman, Nature Materials, 9 (2010) 865-865.

[275] R. Ren, Y. Guo, R. Zhu, Optics Letters, 37 (2012) 4245-4247.

[276] U.W. Paetzold, E. Moulin, B.E. Pieters, R. Carius, U. Rau, Opt. Express, 19 (2011) A1219A1230. 
[277] K. Zhou, Z. Guo, X. Li, J.-Y. Jung, S.-W. Jee, K.-T. Park, H.-D. Um, N. Wang, J.-H. Lee, Opt. Express, 20 (2012) A777-A787.

[278] E. Mulazimoglu, G. Nogay, R. Turan, H. Emrah Unalan, Applied Physics Letters, 103 (2013) 143124.

[279] C. Lin, M.L. Povinelli, Applied Physics Letters, 97 (2010) 071110.

[280] X. Shen, B. Sun, D. Liu, S.T. Lee, J Am Chem Soc, 133 (2011) 19408-19415.

[281] P. Yu, C.-Y. Tsai, J.-K. Chang, C.-C. Lai, P.-H. Chen, Y.-C. Lai, P.-T. Tsai, M.-C. Li, H.-T. Pan, Y.-Y. Huang, C.-I. Wu, Y.-L. Chueh, S.-W. Chen, C.-H. Du, S.-F. Horng, H.-F. Meng, ACS Nano, 7 (2013) 10780-10787.

[282] L. He, C. Jiang, Rusli, D. Lai, H. Wang, Applied Physics Letters, 99 (2011) 021104.

[283] H. Jeong, H. Song, Y. Pak, I.K. Kwon, K. Jo, H. Lee, G.Y. Jung, Advanced materials, 26 (2014) 3445-3450.

[284] H.-J. Syu, S.-C. Shiu, C.-F. Lin, Solar Energy Materials and Solar Cells, 98 (2012) 267-272.

[285] M. Sharma, P.R. Pudasaini, F. Ruiz-Zepeda, D. Elam, A.A. Ayon, ACS Appl Mater Interfaces, 6 (2014) 4356-4363.

[286] K.-T. Park, H.-J. Kim, M.-J. Park, J.-H. Jeong, J. Lee, D.-G. Choi, J.-H. Lee, J.-H. Choi, Scientific Reports, 5 (2015) 12093.

[287] T. Subramani, H.-J. Syu, C.-T. Liu, C.-C. Hsueh, S.-T. Yang, C.-F. Lin, ACS Applied Materials \& Interfaces, 8 (2016) 2406-2415.

[288] Y. Jiang, X. Gong, R. Qin, H. Liu, C. Xia, H. Ma, Nanoscale Research Letters, 11 (2016) 1-7.

[289] Y.-C. Lai, Y.-F. Chang, P.-T. Tsai, J.-k. Chang, W.-H. Tseng, Y.-C. Lin, C.-Y. Hsiao, H.-W. Zan, C.-I. Wu, G.-C. Chi, H.-F. Meng, P. Yu, Opt. Express, 24 (2016) A414-A423.

[290] G. Kalita, S. Adhikari, H.R. Aryal, R. Afre, T. Soga, M. Sharon, W. Koichi, M. Umeno, Journal of Physics D: Applied Physics, 42 (2009) 115104.

[291] M.J. Lo Faro, C. D'Andrea, E. Messina, B. Fazio, P. Musumeci, R. Reitano, G. Franzo, P.G. Gucciardi, C. Vasi, F. Priolo, F. Iacona, A. Irrera, Sci Rep, 5 (2015) 16753.

[292] G. Jia, M. Steglich, I. Sill, F. Falk, Solar Energy Materials and Solar Cells, 96 (2012) 226-230.

[293] G. Fan, H. Zhu, K. Wang, J. Wei, X. Li, Q. Shu, N. Guo, D. Wu, ACS Appl Mater Interfaces, 3 (2011) 721-725.

[294] J. Guobin, A. Gawlik, J. Bergmann, B. Eisenhawer, S. Schonherr, G. Andra, F. Falk, Photovoltaics, IEEE Journal of, 4 (2014) 28-32.

[295] G. Jia, B. Eisenhawer, J. Dellith, F. Falk, A. Thøgersen, A. Ulyashin, The Journal of Physical Chemistry C, 117 (2013) 1091-1096.

[296] Y. Jia, Z. Zhang, L. Xiao, R. Lv, Nanoscale Research Letters, 11 (2016) 1-6.

[297] A.S. Togonal, M. Foldyna, W. Chen, J.X. Wang, V. Neplokh, M. Tchernycheva, J. Nassar, P. Roca i Cabarrocas, Rusli, The Journal of Physical Chemistry C, 120 (2016) 2962-2972.

[298] Z. Junliang, S. Xiao Wei, T. Swee Tiam, Electron Devices, IEEE Transactions on, 56 (2009) 2995-2999.

[299] M. Orita, H. Ohta, M. Hirano, H. Hosono, Applied Physics Letters, 77 (2000) 4166-4168.

[300] M. Yameen, S.K. Srivastava, P. Singh, P. Prathap, Vandana, C.M.S. Rauthan, P.K. Singh, Advanced Science Letters, 20 (2014) 1540-1544.

[301] W.W. He, K.J. Wu, K. Wang, T.F. Shi, L. Wu, S.X. Li, D.Y. Teng, C.H. Ye, Scientific Reports, 4 (2014) 3715.

[302] K.Q. Peng, X. Wang, X.L. Wu, S.T. Lee, Nano Letters, 9 (2009) 3704-3709. 
[303] J.M. Spurgeon, S.W. Boettcher, M.D. Kelzenberg, B.S. Brunschwig, H.A. Atwater, N.S. Lewis, Advanced materials, 22 (2010) 3277-3281.

[304] J. Shi, Y. Hara, C. Sun, M.A. Anderson, X. Wang, Nano Lett, 11 (2011) 3413-3419.

[305] X. Shen, B. Sun, F. Yan, J. Zhao, F. Zhang, S. Wang, X. Zhu, S. Lee, Acs Nano, 4 (2010) 58695876.

[306] J.R. Maiolo, III, B.M. Kayes, M.A. Filler, M.C. Putnam, M.D. Kelzenberg, H.A. Atwater, N.S. Lewis, Journal of the American Chemical Society, 129 (2007) 12346-12347.

[307] A.P. Goodey, S.M. Eichfeld, K.-K. Lew, J.M. Redwing, T.E. Mallouk, Journal of the American Chemical Society, 129 (2007) 12344-12345.

[308] S.W. Boettcher, J.M. Spurgeon, M.C. Putnam, E.L. Warren, D.B. Turner-Evans, M.D. Kelzenberg, J.R. Maiolo, H.A. Atwater, N.S. Lewis, Science, 327 (2010) 185-187.

[309] E.A. Dalchiele, F. Martin, D. Leinen, R.E. Marotti, J. Ramon Ramos-Barrado, Journal of the Electrochemical Society, 156 (2009) K77-K81.

[310] K. Peng, X. Wang, S.-T. Lee, Applied Physics Letters, 92 (2008) 163103.

[311] I. Oh, J. Kye, S. Hwang, Nano Lett, 12 (2012) 298-302.

[312] S. Hu, M.R. Shaner, J.A. Beardslee, M. Lichterman, B.S. Brunschwig, N.S. Lewis, Science, 344 (2014) 1005-1009.

[313] M.R. Shaner, S. Hu, K. Sun, N.S. Lewis, Energy \& Environmental Science, 8 (2015) 203-207.

[314] M.J. Kenney, M. Gong, Y. Li, J.Z. Wu, J. Feng, M. Lanza, H. Dai, Science, 342 (2013) 836-840.

[315] X. Zhou, R. Liu, K. Sun, D. Friedrich, M.T. McDowell, F. Yang, S.T. Omelchenko, F.H. Saadi, A.C. Nielander, S. Yalamanchili, K.M. Papadantonakis, B.S. Brunschwig, N.S. Lewis, Energy \& Environmental Science, 8 (2015) 2644-2649.

[316] K. Sun, F.H. Saadi, M.F. Lichterman, W.G. Hale, H.-P. Wang, X. Zhou, N.T. Plymale, S.T. Omelchenko, J.-H. He, K.M. Papadantonakis, B.S. Brunschwig, N.S. Lewis, Proceedings of the National Academy of Sciences of the United States of America, 112 (2015) 3612-3617.

[317] J. Kim, C.-l. Jung, M. Kim, S. Kim, Y. Kang, H.-s. Lee, J. Park, Y. Jun, D. Kim, Nanoscale, 8 (2016) 7761-7767.

[318] J.P. Alper, M. Vincent, C. Carraro, R. Maboudian, Applied Physics Letters, 100 (2012) 163901. 